\title{
Matter Mixing in Aspherical Core-collapse Supernovae: Three-dimensional Simulations with Single-star and Binary Merger Progenitor Models for SN 1987A
}

\author{
Masaomi Ono ${ }^{1,2}$ (10), Shigehiro Nagataki ${ }^{1,2}$ (1) Gilles Ferrand $^{1,2}$, Koh Takahashi $^{3}$ (ㅁ) Hideyuki Umeda $^{4}$, Takashi Yoshida $^{4}$, \\ Salvatore Orlando ${ }^{5}$, and Marco Miceli $^{5,6}$ (i) \\ ${ }^{1}$ Astrophysical Big Bang Laboratory, RIKEN Cluster for Pioneering Research, 2-1 Hirosawa, Wako, Saitama 351-0198, Japan; masaomi.ono@riken.jp \\ ${ }^{2}$ RIKEN Interdisciplinary Theoretical and Mathematical Science Program (iTHEMS), 2-1 Hirosawa, Wako, Saitama 351-0198, Japan \\ ${ }^{3}$ Max-Planck-Institut für Gravitationsphysik (Albert-Einstein-Institute), Am Mühlenberg 1, D-14476 Potsdam-Golm, Germany \\ ${ }^{4}$ Department of Astronomy, Graduate School of Science, The University of Tokyo, 7-3-1 Hongo, bunkyo-ku, Tokyo 113-0033, Japan \\ ${ }^{5}$ INAF-Osservatorio Astronomico di Palermo, Piazza del Parlamento 1, I-90134 Palermo, Italy \\ ${ }^{6}$ Dipartimento di Fisica e Chimica, Universitá degli Studi di Palermo, Piazza del Parlamento 1, I-90134 Palermo, Italy \\ Received 2019 September 2; revised 2019 November 22; accepted 2019 November 26; published 2020 January 15
}

\begin{abstract}
We perform three-dimensional hydrodynamic simulations of aspherical core-collapse supernovae, focusing on the matter mixing in SN 1987A. The impacts of four progenitor (pre-supernova) models and parameterized aspherical explosions are investigated. The four pre-supernova models include a blue supergiant (BSG) model based on a slow-merger scenario developed recently for the progenitor of SN 1987A (Urushibata et al. 2018). The others are a BSG model based on a single-star evolution and two red supergiant (RSG) models. Among the investigated explosion (simulation) models, a model with the binary merger progenitor model and an asymmetric bipolar-like explosion, which invokes a jetlike explosion, best reproduces constraints on the mass of high-velocity ${ }^{56} \mathrm{Ni}$, as inferred from the observed [Fe II] line profiles. The advantage of the binary merger progenitor model for the matter mixing is the flat and less extended $\rho r^{3}$ profile of the $\mathrm{C}+\mathrm{O}$ core and the helium layer, which may be characterized by the small helium core mass. From the best explosion model, the direction of the bipolar explosion axis (the strongest explosion direction) and the neutron star (NS) kick velocity and direction are predicted. Other related implications and future prospects are also given.
\end{abstract}

Unified Astronomy Thesaurus concepts: Core-collapse supernovae (304); Supernova dynamics (1664); Supergiant stars (1661); Neutron stars (1108); Stellar evolutionary models (2046); Hydrodynamical simulations (767);

Explosive nucleosynthesis (503)

Supporting material: animations

\section{Introduction}

Since the discovery of supernova 1987A (SN 1987A) in the Large Magellanic Cloud on 1987 February 23, more than 30 yr have passed, and it has been in the young supernova remnant phase. So far, there have been many observations of SN 1987A at a wide range of wavelengths (for a review of the observational features of SN 1987A, see, e.g., Arnett et al. 1989b; McCray 1993; McCray \& Fransson 2016). Thus, SN 1987A provides a unique opportunity to investigate the evolution from a supernova to a supernova remnant thanks to its age ( $\sim 30 \mathrm{yr})$ and proximity $(\sim 50 \mathrm{kpc})$. In order to extract information from the observations of the supernova remnant, theoretical modeling of the evolution from the explosion to its supernova remnant is indispensable. In this paper, we theoretically investigate an early evolution of SN 1987A (up to a few days), focusing on the matter mixing and related observables; we have linked the evolution to phases of the supernova remnant (Orlando et al. 2019b).

Large-scale matter mixing has been indicated from observations of SN 1987A as follows. Early detections of hard X-ray emission (Dotani et al. 1987; Sunyaev et al. 1987) and direct $\gamma$-ray lines from the decay of ${ }^{56} \mathrm{Co}$ (Matz et al. 1988; Varani et al. 1990) revealed the existence of radioactive ${ }^{56} \mathrm{Ni}$ in highvelocity outer layers in the expanding ejecta consisting of helium and hydrogen. It is noted that ${ }^{56} \mathrm{Co}$ was the decay product of ${ }^{56} \mathrm{Ni}$ (in the sequence of ${ }^{56} \mathrm{Ni} \rightarrow{ }^{56} \mathrm{Co} \rightarrow{ }^{56} \mathrm{Fe}$ ) that had been synthesized by the explosive nucleosynthesis during the explosion (the half-lives of the sequence, ${ }^{56} \mathrm{Ni} \rightarrow{ }^{56} \mathrm{Co} \rightarrow$ ${ }^{56} \mathrm{Fe}$, are 6.1 and 77 days, respectively; Nadyozhin 1994). The fine structure developed in the $\mathrm{H}_{\alpha}$ line (the so-called Bochum event; Hanuschik et al. 1988) has also implied the existence of clumps of high-velocity $\left(\sim 4700 \mathrm{~km} \mathrm{~s}^{-1}\right){ }^{56} \mathrm{Ni}$ with a few $10^{-3} M_{\odot}$ (Utrobin et al. 1995). Observed emission lines of [Fe II] (18 and $26 \mu \mathrm{m})$ from SN 1987A at $\sim 400$ days after the explosion (Haas et al. 1990) have shown that the tails of the distribution of Doppler velocities reach $\sim 4000 \mathrm{~km} \mathrm{~s}^{-1}$, and the centroids of the lines are redshifted (for 18 and $26 \mu \mathrm{m}$, the centroids are at $450 \pm 200$ and $680 \pm 200 \mathrm{~km} \mathrm{~s}^{-1}$, respectively). It has been interpreted that between $4 \%$ and $17 \%$ of the iron had a high velocity of $\gtrsim 3000 \mathrm{~km} \mathrm{~s}^{-1}$ (Haas et al. 1990). Later observations of [Ni II] lines from SN 1987A at $\sim 640$ days also indicated a similar high velocity of iron $\left(\sim 3000 \mathrm{~km} \mathrm{~s}^{-1}\right.$; Colgan et al. 1994). The spectral modeling of the late phase (200-2000 days) of SN 1987A has revealed inward mixing of hydrogen down to velocities of $\lesssim 700 \mathrm{~km} \mathrm{~s}^{-1}$ (Kozma \& Fransson 1998). Theoretical studies based on one-dimensional (1D) hydrodynamical simulations with radiative transfer have shown that the early appearance of hard X-ray emission and $\gamma$-ray lines and optical light curves cannot be explained without some degree of mixing of ${ }^{56} \mathrm{Ni}$ into fast-moving outer layers (Pinto \& Woosley 1988; Woosley 1988; Shigeyama \& Nomoto 1990). To reproduce the observed optical light curves, artificial mixing of ${ }^{56} \mathrm{Ni}$ up to velocities of $3000-4000 \mathrm{~km} \mathrm{~s}^{-1}$ is necessary (Shigeyama \& Nomoto 1990; Blinnikov et al. 2000). In addition to ${ }^{56} \mathrm{Ni}$, Shigeyama \& Nomoto (1990) and 
Blinnikov et al. (2000) also insisted on inward mixing of hydrogen down to velocities of 800 and $1300 \mathrm{~km} \mathrm{~s}^{-1}$, respectively, although the values are higher than those deduced from the spectral modeling (Kozma \& Fransson 1998).

What is the mechanism of the mixing? Rayleigh-Taylor (RT) instability has been considered to be one of the possible mechanisms of the matter mixing in core-collapse supernovae. The RT unstable condition is described as $\nabla P \cdot \nabla \rho<0$ (Chevalier 1976), where $P$ is the pressure and $\rho$ is the density. 1D hydrodynamical simulations with a progenitor model for SN 1987A have shown that the unstable condition could be realized during the shock propagation around the interface between the $\mathrm{C}+\mathrm{O}$ core and the helium layer $(\mathrm{C}+\mathrm{O} / \mathrm{He})$ and the one between the helium and hydrogen layers $(\mathrm{He} / \mathrm{H}$; Ebisuzaki et al. 1989; Benz \& Thielemann 1990).

Motivated by the observational evidence of the matter mixing in SN 1987A, multidimensional hydrodynamical simulations of the propagation of the supernova shock wave have been performed, focusing on the development of RT instabilities (Arnett et al. 1989a; Hachisu et al. 1990, 1992; Fryxell et al. 1991; Herant \& Benz 1991, 1992; Mueller et al. 1991). All of the studies have assumed spherical symmetry in the explosions, and among the investigated models, the obtained maximum velocity of ${ }^{56} \mathrm{Ni}$ is only $\sim 2000 \mathrm{~km} \mathrm{~s}^{-1}$ (Herant \& Benz 1991, 1992). Hence, aspherical explosions that had not been considered in those studies above might be necessary to explain the observations.

Recent observations of emission lines of [Si I $]+[\mathrm{Fe}$ II $]$ and He I (1.644 and $2.058 \mu \mathrm{m}$, respectively) by the Hubble Space Telescope (HST) and Very Large Telescope (Kjær et al. 2010; Larsson et al. 2013, 2016) have revealed that the threedimensional (3D) morphology of the inner ejecta of SN 1987A is globally elliptical/elongated (the ratio of the major to minor axes of the inner ejecta is $1.8 \pm 0.17$; Kjær et al. 2010). It is known that in the nebula around SN 1987A, there is a triple ring structure consisting of an inner equatorial ring (ER) and two outer rings (ORs), and the configurations with respect to the Earth have been deduced (e.g., Sugerman et al. 2005a; Tziamtzis et al. 2011). The optical spectroscopy of the light echoes of SN 1987A has also indicated asymmetries in the line profiles of $\mathrm{H}_{\alpha}$ and Fe II, which is consistent with the elongated ejecta and two-sided distribution of ${ }^{56} \mathrm{Ni}$ (Sinnott et al. 2013). New spots and diffuse emission outside the ER found by more recent $H S T$ observations may provide additional insights into the evolution of the ER and ejecta (Larsson et al. 2019). Recent observations of spatially resolved 3D distributions of the rotational transition lines of $\mathrm{CO}$ and $\mathrm{SiO}$ molecules by the Atacama Large Millimeter/submillimeter Array (ALMA) have indicated that the distributions are clumpy and not spherical at all (Abellán et al. 2017). Further observations by ALMA have revealed that dust emission from the inner ejecta is also clumpy and asymmetric (Cigan et al. 2019).

Direct emission lines from the decay of long-lived radioisotope ${ }^{44} \mathrm{Ti}$ (the half-life of the decay sequence, ${ }^{44} \mathrm{Ti} \rightarrow{ }^{44} \mathrm{Sc} \rightarrow{ }^{44} \mathrm{Ca}$, is $58.9 \pm 0.3 \mathrm{yr}$; Ahmad et al. 2006), which was the product of the explosive nucleosynthesis, have been observed in SN 1987A (Grebenev et al. 2012; Boggs et al. 2015). The initial mass of ${ }^{44} \mathrm{Ti}$ was estimated as $(3.1 \pm 0.8) \times 10^{-4} M_{\odot}$ in Grebenev et al. (2012). Recent observations by NuSTAR (Boggs et al. 2015) have also revealed that ${ }^{44} \mathrm{Ti} \gamma$-ray lines have been redshifted with a velocity of $700 \pm 400 \mathrm{~km} \mathrm{~s}^{-1}$, which invokes a large-scale asymmetry in the explosion of SN 1987A, and the initial mass of ${ }^{44} \mathrm{Ti}$ was estimated as $(1.5 \pm 0.3) \times 10^{-4} M_{\odot}$ (Boggs et al. 2015).
Despite searching for more than $30 \mathrm{yr}$, the compact object of SN 1987A has not been detected yet. From the fact that there has been no detection from millimeter, near-infrared, optical, ultraviolet, and X-ray observations, several constraints on the compact object have been argued, and it has been inferred that the compact object is a thermally emitting neutron star (NS) obscured by dust (Orlando et al. 2015; Alp et al. 2018), although the possibility that the compact object is an NS with nonthermal emission was also argued (Esposito et al. 2018). Meanwhile, X-ray observations of nearby core-collapse supernova remnants, e.g., Cassiopeia A (Cas A), have revealed that the direction of an NS's motion relative to the explosion center is the opposite of the gaseous intermediate elements in the supernova ejecta, and it has been inferred that the NS kicks stem from asymmetric explosive mass ejections (Katsuda et al. 2018). Theoretically, NS kicks are expected by neutrino-driven core-collapse supernova explosions thanks to their aspherical nature (Wongwathanarat et al. 2010, 2013). Recent ALMA observations of dust emission from the ejecta of SN 1987A have insisted that a dust peak found to the northeast of the center of the remnant could be an indirect detection of the compact object (Cigan et al. 2019).

The mechanisms of core-collapse supernova explosions have not been elucidated yet. Theoretically, it has been considered that a canonical core-collapse supernova could be triggered by the delayed neutrino heating aided by convection (Herant et al. 1994) and/or standing accretion shock instability (SASI; Blondin et al. 2003), where multidimensional effects are essential (for a review of the mechanism of core-collapse supernovae, see Janka 2012; Janka et al. 2012; Kotake et al. 2012a, 2012b; Burrows 2013; Müller 2016). Hitherto, based on the delayed neutrino heating mechanism, many two-dimensional (2D) and 3D hydrodynamical simulations with an approximate neutrino transport have been performed for a few decades (Burrows et al. 1995a; Kifonidis et al. 2003, 2006; Scheck et al. 2006, 2008; Marek \& Janka 2009; Nordhaus et al. 2010b; Suwa et al. 2010; Kuroda et al. 2012; Müller et al. 2012a, 2012b, 2012c, 2017; Takiwaki et al. 2012, 2014; Bruenn et al. 2013, 2016; Couch \& Ott 2013; Dolence et al. 2013; Hanke et al. 2013; Ott et al. 2013; Wongwathanarat et al. 2013; Couch \& O'Connor 2014; Nakamura et al. 2014; Couch $\&$ Ott 2015; Pan et al. 2016; Radice et al. 2016, 2017; Nagakura et al. 2017; Burrows et al. 2019; Vartanyan et al. 2019). Since the involved physical effects, e.g., neutrino transport, general relativity, and nuclear equation of state (EOS), are rather complicated and multidimensional, ab initio hydrodynamical simulations of core-collapse supernovae are rather demanding in the viewpoint of numerical costs; the adopted physical effects and their approximations, in particular for the neutrino transport, have been rather varied among simulations, and a consensus has not yet been reached. Actually, comparisons of the results between 2D and 3D simulations have been made, and the explodabilities in $3 \mathrm{D}$ relative to those in 2D are controversial (Nordhaus et al. 2010b; Dolence et al. 2013; Hanke et al. 2013; Takiwaki et al. 2014). It is noted that a strong sloshing mode $(l=1)$ of SASI seen in 2D, which makes an asymmetric dipolar morphology of the shock, tends to be less evident in 3D at later phases of the shock revival (Nordhaus et al. 2010b; Hanke et al. 2013).

On the other hand, magnetohydrodynamical (MHD) simulations of core-collapse supernovae have demonstrated jetlike magnetorotationally driven explosions (Kotake et al. 2004; 
Sawai et al. 2005; Burrows et al. 2007; Takiwaki et al. 2009; Sawai et al. 2013; Mösta et al. 2014; Sawai \& Yamada 2016). For a successful launch of a jet, generally, both strong magnetic field and rapid rotation before the core collapse are necessary; however, it has not yet been unveiled from which evolutionary paths both conditions are fulfilled (for an example of stellar evolution calculations of a single massive star with magnetic field, see, e.g., Heger et al. 2005). The magnetorotational instability (Balbus \& Hawley 1998) could play a significant role in the amplification of the magnetic field during the core collapse and shock revival, but high-resolution simulations are necessary to capture the fastest-growing mode. and it is difficult to assess its role by global hydrodynamical simulations. See, e.g., Sawai \& Yamada (2016) for an attempt to investigate the impact of the magnetorotational instability on core-collapse supernovae. The possibility of jittering jets explosion mechanism was also argued for SN 1987A (Bear \& Soker 2018).

In the context of matter mixing in core-collapse supernovae, the possible effects of aspherical core-collapse supernova explosions have been investigated based on multidimensional hydrodynamic simulations. The effects of mildly jetlike explosions on matter mixing have been studied based on 2D hydrodynamical simulations with a progenitor model for SN 1987A (Yamada \& Sato 1991; Nagataki et al. 1998b; Nagataki 2000). Nagataki et al. (1998b) and Nagataki (2000) obtained high-velocity ${ }^{56} \mathrm{Ni}$ corresponding to the tails (up to $\sim 3000 \mathrm{~km} \mathrm{~s}^{-1}$ ) of [Fe II] lines with a large amplitude (30\%) of perturbations in velocities at the phase when the shock wave reaches the $\mathrm{He} / \mathrm{H}$ interface. In the context of jetlike explosions, Nagataki et al. (1997, 1998a) suggested that a jetlike explosion enhances the amount of ${ }^{44} \mathrm{Ti}$ synthesized by the explosive nucleosynthesis thanks to a strong alpha-rich freeze-out. Hungerford et al. $(2003,2005)$ investigated the effects of jetlike and single-lobe explosions on the $\gamma$-ray lines using a 3D smoothed particle hydrodynamical (SPH) code. Kifonidis et al. (2000, 2003, 2006) investigated matter mixing with more realistic explosion models based on 2D high-resolution hydrodynamical simulations (with adaptive mesh refinement (AMR)) of neutrino-driven core-collapse supernovae aided by convection and/or SASI. The authors found that a globally aspherical explosion dominated by low-order unstable modes $(l=1,2)$ with an explosion energy of $2 \times 10^{51}$ erg produces high-velocity ${ }^{56} \mathrm{Ni}$ clumps $\left(\sim 3300 \mathrm{~km} \mathrm{~s}^{-1}\right.$; Kifonidis et al. 2006). Joggerst et al. (2009, 2010a, 2010b) studied the development of RT instabilities in spherical core-collapse supernovae of solarmetallicity, metal-poor, and zero-metallicity massive stars based on $2 \mathrm{D}$ and $3 \mathrm{D}$ hydrodynamical simulations. If a star ends its life as a compact BSG, the mixing by RT instability is significantly reduced, and fallback is enhanced compared with those of RSGs. Thus, the structure of the progenitor star could be essential for matter mixing. Ellinger et al. (2012) studied RT mixing in a series of aspherical core-collapse supernova explosions using a 3D SPH code, and the sizes of the arising clumps were studied based on Fourier transformations.

The effects of the dimensionality of hydrodynamical simulations on the matter mixing in core-collapse supernovae have been controversial. The growth of RT instabilities in 3D simulations of a spherical supernova explosion is faster than that in corresponding 2D simulations, but the widths of the mixed regions at the time of the saturation are similar in $2 \mathrm{D}$ and 3D in the end (Joggerst et al. 2010b). On the other hand, Hammer et al. (2010) demonstrated an effective mixing in 3D due to the faster growth of RT fingers and less deceleration of metal-rich clumps compared with that in the corresponding 2D simulation. Generally, the resolutions of 2D hydrodynamical simulations can be higher than those of 3D ones; however, axisymmetric 2D Eulerian hydrodynamical simulations could introduce numerical artifacts around the polar axis (Gawryszczak et al. 2010). In keeping with the different behaviors between 2D and $3 \mathrm{D}$ and the defect of possible numerical artifacts in axisymmetric 2D simulations, 3D high-resolution simulations are necessary for a study of matter mixing.

In our previous papers (Ono et al. 2013; Mao et al. 2015, hereafter Paper I and Paper II, respectively), we have systematically investigated the matter mixing in SN 1987A based on 2D hydrodynamical simulations with an AMR code with an ad hoc way of the initiation of explosions. In Paper I, we parametrically explored the impact of mildly aspherical explosions with a clumpy structure on the distribution of the radial velocities of ${ }^{56} \mathrm{Ni}$ and the line-of-sight velocity distribution of ${ }^{56} \mathrm{Ni}$, which corresponds to the observed velocity profiles of [Fe II] lines. It was found that the maximum velocity of ${ }^{56} \mathrm{Ni}$ is at most $\sim 3000 \mathrm{~km} \mathrm{~s}^{-1}$. In Paper II, the possible effects of large perturbations in the density of the progenitor star were explored, and at most, $\sim 4000 \mathrm{~km} \mathrm{~s}^{-1}$ of ${ }^{56} \mathrm{Ni}$ can be obtained by asymmetric bipolar explosions with radially coherent perturbations (amplitude of 50\%) in the density of the progenitor star. The obtained line-of-sight velocity distribution of ${ }^{56} \mathrm{Ni}$, however, seems to be different from those of the observed [Fe II] line profiles.

Wongwathanarat et al. (2015) investigated the dependence of matter mixing on progenitor models based on 3D hydrodynamical simulations of neutrino-driven core-collapse supernovae from the shock revival to the shock breakout. It was found that the extent of mixing depends sensitively on the density structure of the progenitor model, i.e., the sizes of the $\mathrm{C}$ $+\mathrm{O}$ core and helium layer and the density gradient at the $\mathrm{He} / \mathrm{H}$ interface. In red supergiant (RSG) models, high-velocity ${ }^{56} \mathrm{Ni}$ of $4000-5000 \mathrm{~km} \mathrm{~s}^{-1}$ is obtained. In a $15 M_{\odot}$ blue supergiant (BSG) model, relatively high-velocity ${ }^{56} \mathrm{Ni}\left(\sim 3500 \mathrm{~km} \mathrm{~s}^{-1}\right)$ is obtained. On the other hand, in a $20 M_{\odot}$ BSG model, the maximum ${ }^{56} \mathrm{Ni}$ velocity is only $\sim 2200 \mathrm{~km} \mathrm{~s}^{-1}$ because of the strong deceleration of inner ejecta by the reverse shock and insufficient time for the growth of RT instabilities at the $\mathrm{He} / \mathrm{H}$ interface. Utrobin et al. (2015) modeled optical light curves based on part of the 3D hydrodynamical models above. Among the investigated models, only one BSG model reproduces the dome-like shape of the light-curve maximum of SN 1987A. As the authors mentioned, the mass of the helium core of the progenitor model is, however, only $\sim 4 M_{\odot}$, which is less than the value for the progenitor star of SN 1987A $\left(6 \pm 1 M_{\odot}\right.$; Arnett et al. 1989b; see below for details).

The properties of the progenitor star of SN 1987A have been obtained from observations (for a review, see Arnett et al. 1989b). The progenitor was identified as a compact B3 Ia BSG, Sanduleak $-69^{\circ} 202$ (hereafter Sk $-69^{\circ} 202$; Walborn et al. 1987; West et al. 1987). The estimated intrinsic bolometric magnitude is translated into a luminosity of (3-6) $\times 10^{38} \mathrm{erg}$ $\mathrm{s}^{-1}$. The effective temperature is $\sim 16,000 \mathrm{~K}$ (Humphreys \& McElroy 1984), with a probable range of $15,000-18,000 \mathrm{~K}$ (Arnett et al. 1989b). From models of massive stars, the helium core mass of Sk $-69^{\circ} 202$ could be in the range of $6 \pm 1 M_{\odot}$ (e.g., Woosley 1988). Another notable feature related to the progenitor of SN 1987A is the triple ring structure discovered 
around $\mathrm{Sk}-69^{\circ} 202$ after the supernova event (Wampler et al. 1990; Burrows et al. 1995b), which invokes an axisymmetric but nonspherical mass ejection during the stellar evolution. The expansion velocities of the rings, the inner ER and two ORs, have been deduced as $\sim 10$ and $\sim 26 \mathrm{~km} \mathrm{~s}^{-1}$, respectively (Crotts \& Heathcote 1991, 2000), which is consistent with the wind velocities of RSGs; it has been interpreted that the three rings were ejected at least $\sim 20,000 \mathrm{yr}$ ago, i.e., Sk $-69^{\circ} 202$ could have been an RSG about 20,000 yr ago (Crotts \& Heathcote 1991; Burrows et al. 1995b). Additionally, anomalous abundances of helium and CNO-processed elements in the circumstellar material including the rings have been reported from observations of emission lines, i.e., $\mathrm{He} / \mathrm{H}$ (number ratio) $=0.25 \pm 0.05$ (Lundqvist \& Fransson 1996), $\mathrm{He} / \mathrm{H}=0.17 \pm 0.06$ (Mattila et al. 2010), $\mathrm{He} / \mathrm{H}=0.14 \pm 0.06$ (France et al. 2011), N/C =7.8 \pm 4 (Fransson et al. 1989), $\mathrm{N} / \mathrm{C}=5.0 \pm 2.0$ (Lundqvist \& Fransson 1996), $\mathrm{N} / \mathrm{O}=1.6 \pm$ 0.8 (Fransson et al. 1989), $\mathrm{N} / \mathrm{O}=1.1 \pm 0.4$ (Lundqvist \& Fransson 1996), and $\mathrm{N} / \mathrm{O}=1.5 \pm 0.7$ (Mattila et al. 2010). These abundance ratios indicate an enhancement of material that underwent hydrogen burning through the CNO cycle in the nebula. The problem, however, is how the products of the hydrogen burning had been mixed into the hydrogen envelope and the nebula in the end.

Hitherto, there have been many attempts to construct singlestar evolution models that satisfy at least a part of the requirements for $\mathrm{Sk}-69^{\circ} 202$ mentioned above. A major issue in single-star models, however, is that extreme fine tuning of parameters related to specific assumptions, e.g., reduced metallicity (Arnett et al. 1989b), enhancements of the mass loss and helium abundance in the hydrogen envelope (Saio et al. 1988a), restricted convection (Woosley et al. 1988), and rotationally induced mixing (Weiss et al. 1988), is necessary in order for the progenitor to end as a BSG and/or obtain the abundance anomalies. Another unignorable issue in single-star scenarios is how the triple ring nebula could be formed in this context. If a progenitor star is rapidly rotating, the envelope could obtain considerable angular momentum by a spin-up mechanism (Heger \& Langer 1998). Chita et al. (2008) performed 2D hydrodynamical simulations of the evolution of the wind nebula of a $12 M_{\odot}$ star with a blue loop (red-bluered evolution) in the Hertzsprung-Russell (H-R) diagram, and the formation of a triple ring structure was demonstrated during the blue phase thanks to the spin-up mechanism (Heger \& Langer 1998). The star, however, ends its life as an RSG. To date, there has been no single-star model that satisfies all of the observational features of $\mathrm{Sk}-69^{\circ} 202$ (for reviews on the progenitor of SN 1987A, see Arnett et al. 1989b; Podsiadlowski 1992; Smartt 2009).

On the other hand, evolution models for Sk $-69^{\circ} 202$ based on binary mergers through a common-envelope interaction have been proposed (Podsiadlowski et al. 1990, 1992; see Hillebrandt \& Meyer 1989, for a related common-envelope model) as alternative (and probably more natural) explanations of the red-to-blue evolution, the abundance anomalies in the nebula, and the formation of the triple ring nebula (for the overall binary merger scenario, see Section 2.2). Along this scenario, Ivanova et al. (2002) demonstrated the penetration of the material from the secondary into the core of the primary based on 2D hydrodynamical simulations. Later, Morris \& Podsiadlowski (2007, 2009) successfully reproduced the formation of a triple ring structure very similar to the observed one based on 3D SPH simulations. Recently, progenitor models for SN 1987A based on the binary merger scenario have been developed by two independent groups (Menon \& Heger 2017; Urushibata et al. 2018). They successfully found appropriate models that satisfy all of the observational features of Sk $-69^{\circ}$ 202 mentioned above. Compared with Menon \& Heger (2017), Urushibata et al. (2018) included the effects of the spin-up of the envelope due to the angular momentum transfer from the orbit. Additionally, recent light-curve modeling for SN 1987A (Menon et al. 2019) based on the binary merger models (Menon \& Heger 2017) has shown that the models better fit to the observed optical light curves than single-star models. Recently, direct $\gamma$-rays from the decay of ${ }^{56} \mathrm{Ni}$ and the scattered $\mathrm{X}$-rays have been theoretically investigated based on 3D hydrodynamical models of neutrino-driven core-collapse supernovae with some binary merger progenitor models (Alp et al. 2019).

In the context of matter mixing in SN 1987A, the studies that have obtained high-velocity ${ }^{56} \mathrm{Ni}\left(\gtrsim 3000 \mathrm{~km} \mathrm{~s}^{-1}\right)$ have investigated only single progenitor star models. Kifonidis et al. (2006), Hammer et al. (2010), and Wongwathanarat et al. (2015) used $15 M_{\odot}$ BSG model B15 (Woosley et al. 1988; denoted as W15 in Sukhbold et al. 2016) to obtain the highvelocity ${ }^{56} \mathrm{Ni}$, but the luminosity of the pre-supernova model is outside the observational constraints. Whereas, with a BSG model (Nomoto \& Hashimoto 1988; Shigeyama \& Nomoto 1990) corresponding to the main-sequence mass of $20 \mathrm{M}_{\odot}$ (denoted as N20 in Wongwathanarat et al. 2015), only a lower velocity of ${ }^{56} \mathrm{Ni}\left(\lesssim 3000 \mathrm{~km} \mathrm{~s}^{-1}\right)$ has been achieved (e.g., Paper I; Wongwathanarat et al. 2015), although the model satisfies the final position in the H-R diagram. ${ }^{7}$ Hitherto, there has been no consistent hydrodynamical model that explains the observed high-velocity ${ }^{56} \mathrm{Ni}$ with a single progenitor star model that fulfills all of the observational requirements for $\mathrm{Sk}-69^{\circ}$ 202. Recently, Utrobin et al. (2019) revisited the modeling of light curves for a larger variety of BSG models than that in Utrobin et al. (2015); it was confirmed that there is no singlestar model that matches all observational features. Therefore, it is worth revisiting the matter mixing in $\mathrm{SN} 1987 \mathrm{~A}$ with a binary merger model.

Motivated by recent observations of the supernova remnant of SN 1987A, 3D hydrodynamical/MHD simulations of the interaction of the expanding ejecta with the ER have been performed, focusing on the X-ray and/or radio emission (Potter et al. 2014; Orlando et al. 2015, 2019a). Recently, Miceli et al. (2019) compared the 3D hydrodynamical model (Orlando et al. 2015) with observed X-ray spectra of the remnant of SN 1987A. Although the morphology of the inner ejecta of SN 1987A is obviously nonspherical (e.g., Larsson et al. 2016), in those studies, spherical symmetry has been assumed in the explosions, and no realistic stellar evolution model has been used. In order to maximize the information that can be extracted by comparing theories with observations of the remnant, 3D hydrodynamical models of aspherical explosions with a realistic stellar evolution model are imperative.

The purpose of this paper is to investigate the impact of progenitor models and parameterized aspherical explosions on the matter mixing in SN 1987A and related observational outcomes, in particular the line-of-sight velocity distribution of ${ }^{56} \mathrm{Ni}$ corresponding to the [Fe II] line profiles, which may

\footnotetext{
7 For the positions of the two BSG models in the H-R diagram, see the points denoted as W15 and N20 in Figure 2 in Sukhbold et al. (2016).
} 
provide nontrivial information on the morphology of the inner ejecta and the configuration relative to the triple ring nebula. In order to accomplish this, we perform 3D hydrodynamical simulations of core-collapse supernova explosions with four pre-supernova models; two are BSG models, and the other two are RSG models. It is noted that a recent binary merger BSG model (Urushibata et al. 2018) is adopted for the study of the matter mixing for the first time. First, we perform many lowerresolution simulations to explore a wide range of parameters related to the asphericities of the explosion and the progenitor dependence. In Paper II, the impact of large-density perturbations in the progenitor star was investigated; however, in order to focus on the purpose above, such effects are not considered in this paper. As a result, we find the best parameter set related to aspherical explosions, and with the parameter set, highvelocity ${ }^{56} \mathrm{Ni}$ of $\sim 4000 \mathrm{~km} \mathrm{~s}^{-1}$ is obtained with the binary merger model. Then, regarding the best parameter set as a fiducial one, we discuss the parameter and progenitor model dependences. Next, fixing the parameter set as the fiducial one, we perform two high-resolution simulations for the two BSG progenitor models, and the differences between the two models are presented. We have used the results of part of the models in this paper as the initial conditions for 3D MHD simulations of the later evolution of SN 1987A (Orlando et al. 2019b), which is a natural extension of our previous studies on spherical explosions for SN 1987A (Orlando et al. 2015, 2019a; Miceli et al. 2019).

This paper is organized as follows. Section 2 is dedicated to the description of the method of computation and the initial conditions. In Section 3, the models and related parameters are delineated. In Section 4, the results of 1D and 3D simulations are presented. Section 5 is devoted to a discussion on related topics. Finally, the study in this paper is summarized in Section 6.

\section{Method and Initial Conditions}

In this section, the numerical method for hydrodynamical simulations and the initial conditions, including the presupernova models, are described in detail.

\subsection{Numerical Method}

In this paper, 3D hydrodynamic simulations of core-collapse supernova explosions are performed. The method is based on our previous papers, Papers I and II, on the matter mixing with 2D hydrodynamic simulations. Here we briefly summarize the method and stress points that are different from the previous ones. The numerical code is FLASH (Fryxell et al. 2000), as in our previous papers (Papers I and II). In this paper, the 3D Cartesian coordinates $(x, y, z)$ are adopted, whereas in Papers I and II, the 2D spherical coordinates $(r, \theta)$ were adopted.

In the simulation, we do not follow the process from the core collapse to a successful shock revival, but the shock-wave propagation from around the interface between the Fe core and the Si layer $(\sim 1000 \mathrm{~km})$ to a radius $\left(\gtrsim 10^{14} \mathrm{~cm}\right)$ larger than the stellar one $\left(\sim 10^{12}-10^{13} \mathrm{~cm}\right)$ is followed. In order to follow such a large difference of the spatial scales, the computational domain is gradually expanded as the shock wave propagates outward. The computational domain is initially set to be $-5000 \mathrm{~km} \leqslant x, \quad y, \quad z \leqslant 5000 \mathrm{~km}$, i.e., $x_{\min }, y_{\min }, z_{\min }=$ $-5000 \mathrm{~km}$ and $x_{\max }, y_{\max }, z_{\max }=5000 \mathrm{~km}$. First, the physical quantities of a pre-supernova model (see Section 2.2) are mapped to the computational domain so that the center of the star is at the origin of the coordinates. When the shock wave approaches the computational boundaries, the simulation is stopped once, and the domain is expanded by a factor of 1.2 for each dimension $\left(x_{\min }\right.$, $y_{\min }, z_{\min }, x_{\max }, y_{\max }$, and $z_{\max }$ are all multiplied by a factor of 1.2 ) as in Papers I and II (in Papers I and II, computational domains are expanded only in the radial direction; i.e., $r_{\max }$ is multiplied by a factor of 1.2). Then the physical quantities are remapped to the new (expanded) computational domain. During the remapping process, in the cells not covered in the previous simulation, the quantities of either the pre-supernova model (see Section 2.2) or the profile of the ambient matter are mapped, depending on the radius. If the cells correspond to the ambient matter, the profile of a spherical steady stellar wind is mapped, where the density profile follows $\rho(r) \propto r^{-2}$ and the mass-loss rate and wind velocity adopted are $\dot{M}_{\text {wind }}=10^{-7} M_{\odot} \mathrm{yr}^{-1}, v_{\text {wind }}=500 \mathrm{~km} \mathrm{~s}^{-1}$, respectively, as in Morris \& Podsiadlowski (2007). After the remapping process, the simulation is restarted again; to cover the large spatial scales, about 75 remappings are necessary.

Explosions are initiated by artificially injecting thermal and kinetic energies around the interface between the Fe core and the Si layer of the mapped pre-supernova profile. The total injected energy, $E_{\mathrm{in}}$, is an initial parameter of the models. The ratio of the injected thermal energy to the kinetic energy is set to be unity. The range of the values of $E_{\text {in }}$ is $(1.5-3.0) \times$ $10^{51}$ erg (see Section 3.1 for the range). It is noted that $E_{\mathrm{in}}$ is not the explosion energy, $E_{\text {exp }}$, which should be obtained as a result of the simulation (see Equation (6) for the definition of $E_{\exp }$ and Table 3 for the obtained values of the explosion energy). In this paper, we consider aspherical explosions, which are obtained by distributing initial radial velocities in nonspherical ways. As such nonspherical explosions, bipolarlike explosions along the $z$-axis (polar axis) with asymmetries across the $x-y$ plane (equatorial plane) are considered, where fluctuations in the initial radial velocities for making clumpy structures are also taken into account. For the details of the description of the distributions of initial radial velocities, see Appendices A and B.

The inner regions centered at the origin that correspond to a compact object (could be a proto-neutron star) are excluded from the cells to be solved. The size of the inner regions corresponding to the compact object is kept as larger than either 0.005 times $x_{\max }$ or three times $\Delta x$ ( $\Delta x$ is the size of the inner cells), whichever is larger, along each dimension. The excluded cells are treated as a boundary condition (BC); i.e., the physical quantities on the cells are replaced to meet the BC to adjacent cells at every time step. During an early phase of the simulation "reflection," a BC is adopted for the excluded cells, and later it is switched to a "diode" BC, as in Papers I and II. The timing of the switching is arbitrary, but it should not affect the major results (see Paper I for details). The mass initially in the excluded cells is regarded as a point mass at the origin, and masses flowing into the excluded cells are added to the point mass at every time step. In the simulation, the point-mass gravity and spherically symmetric self-gravity are taken into account. The former is the gravity due to the time-dependent point mass, and the latter is obtained from the spherically averaged density profiles.

In order to reduce the computational costs, the resolution of the computational grids is adaptively refined (the method is 
called AMR) with the PARAMESH (MacNeice et al. 2000) package implemented in the FLASH code. For lowerresolution simulations in this paper, the maximum and minimum refinement levels are initially set to be 7 and 5, respectively. For high-resolution simulations, the initial maximum and minimum refinement levels are 8 and 5, respectively. If the maximum refinement level is $n$, at most $2^{(n-1)}$ blocks can be created for each dimension. Here the number of grid points in one block for each dimension is eight. Then, the effective resolution of the lower- (higher-) resolution simulations is $\left[2^{6} \times 8\right]^{3}=512^{3}\left(\left[2^{7} \times 8\right]^{3}=1024^{3}\right)$. Since a simulation with the maximum refinement level for all computational regions is rather demanding from the point of view of the numerical cost, in order to reduce the cost, we manually control the regions where the maximum refinement is allowed. The regions around the forward shock (FS) should be solved at the highest resolution because the regions are numerically severe and must be solved by a shock-capturing scheme and dominate the overall dynamics due to their high fluid velocities. Additionally, of interest are regions where instabilities develop, and actually, in the regions around the FS, RT instabilities first start to grow. Then, for both lower- and high-resolution simulations, regions only around the FS are allowed to be at the maximum refinement (as mentioned later, starting immediately before the shock breakout, the maximum refinement level is increased, and the regions allowed to be at the maximum refinement are changed), i.e., the effective resolutions of other regions for lower- and high-resolution simulations are $256^{3}$ and $512^{3}$, respectively. The FS surface (FS radius, $r_{\mathrm{FS}}$ ) is approximately traced at every time step by searching for the cell that has the maximum radial velocity along each radial direction. The regions of $r_{\mathrm{FS}}-0.05 x_{\max } \leqslant r \leqslant r_{\mathrm{FS}}+0.075 x_{\max }$ are allowed to be at the maximum refinement. After the shock breakout, the FS is accelerating rapidly due to the steep pressure gradients, whereas the inner ejecta (originally inside the He core) is left far behind the FS. Then, the complex structures of the inner ejecta introduced at earlier phases are numerically lost after the shock breakout without a special treatment for the refinement. Therefore, starting just before the shock breakout, the maximum refinement levels in the inner regions are increased. In the inner regions of approximately $r \leqslant x_{\max } / 8$, the maximum refinement levels are set to be 8 (effective res.: $1024^{3}$ ) and 9 (effective res.: $2048^{3}$ ) for lower- and highresolution simulations, respectively. In the regions of approximately $x_{\max } / 8 \leqslant r \leqslant x_{\max } / 4$ or around the FS, the maximum refinement levels are set to be 7 (effective res.: $512^{3}$ ) and 8 (effective res.: $1024^{3}$ ) for lower- and high-resolution simulations, respectively. The resolutions of other regions are the same as before the shock breakout.

Since the density and pressure of the ambient matter are rather small compared with those in the expanding ejecta, the shape of the FS is affected by the grid structure of the Cartesian coordinates after the shock breakout; i.e., the shape of the FS tends to be like a square. In order to reduce such numerical artifacts on the shape of the FS, starting just before the shock breakout, the system is rotated by an arbitrary angle about each axis during the remapping process; after that, all of the physical quantities are remapped. The angles are randomly determined within the range from $-\pi / 2$ to $\pi / 2$ for each axis. Due to the randomness of the selection of the arbitrary rotation angles, the effects of the grid structure of the Cartesian coordinate are washed out after several remappings. Actually, we confirmed that the shape of the FS becomes more roundish (natural) than that without such rotations. Since the rotations affect only the outermost ejecta (mostly composed of hydrogen) after the shock breakout, the main results of this paper (the spatial distribution of metals and their velocities), except for the shape of the FS, should not change with or without the rotations.

As in Paper I, perturbations of pre-supernova origin are taken into account in the simulation. When the shock wave reaches around the composition interfaces of $\mathrm{C}+\mathrm{O} / \mathrm{He}$ and $\mathrm{He} / \mathrm{H}$, perturbations of the amplitude of $5 \%$ are introduced in the radial velocities. The perturbations are functions of the angular position $(\theta, \phi)$. We take $l+1$ sampling points for random numbers along the $\theta$ direction at $\theta=0, \pi / l, 2 /(l-1)$, $\ldots, \pi$ and $m+1$ sampling points along the $\phi$ direction at $\theta=0, \pi / m, 2 /(m-1), \ldots, 2 \pi$, where $l$ and $m$ are integers, and $l=128$ and $m=256$ are adopted. Then, at each sampling point, one random number is assigned. A factor for the perturbations to the radial velocities at an angular position $(\theta, \phi)$ is obtained by $1+\epsilon \operatorname{rand}(\theta, \phi)$, where $\epsilon$ is the parameter for the amplitude of the perturbations and set to be $5 \%$, and $\operatorname{rand}(\theta, \phi)$ is a function of the angular position $(\theta, \phi)$ obtained by the interpolation of the assigned random numbers of the adjacent sampling points around the effective angular position $\left(\theta, \phi^{\prime}\right) \equiv(\theta, \phi \sin \theta){ }^{8}$ In this paper, we do not discuss the impact of the perturbations of pre-supernova origins (for the impact, see Paper I).

As in Papers I and II, the explosive nucleosynthesis is taken into account with a small approximate nuclear reaction network (Weaver et al. 1978) coupled with the FLASH code. Elements $n, p,{ }^{1} \mathrm{H},{ }^{3} \mathrm{He},{ }^{4} \mathrm{He},{ }^{12} \mathrm{C},{ }^{14} \mathrm{~N},{ }^{16} \mathrm{O},{ }^{20} \mathrm{Ne},{ }^{24} \mathrm{Mg},{ }^{28} \mathrm{Si},{ }^{32} \mathrm{~S}$, ${ }^{36} \mathrm{Ar},{ }^{40} \mathrm{Ca},{ }^{44} \mathrm{Ti},{ }^{48} \mathrm{Cr},{ }^{52} \mathrm{Fe},{ }^{54} \mathrm{Fe}$, and ${ }^{56} \mathrm{Ni}$ are included. The feedback from the nuclear energy generation is also taken into account. The advection of elements is followed by solving an advection equation for the mass fraction of each element (see Paper I for details).

At early phases of the simulation, the Helmholtz EOS (Timmes \& Swesty 2000), which includes contributions from the radiation, completely ionized ions, and degenerate/ relativistic electrons and positrons, is used. The EOS can cover the physical regions of $10^{-10} \mathrm{~g} \mathrm{~cm}^{-3}<\rho<10^{11} \mathrm{~g} \mathrm{~cm}^{-3}$ and $10^{4} \mathrm{~K}<T<10^{11} \mathrm{~K}$. For a later phase, when $\rho \lesssim 10^{-8} \mathrm{~g} \mathrm{~cm}^{-3}$, another EOS that consists of ideal gas of fully ionized ions, electrons, and radiation is used. For a transition region of $10^{-8} \mathrm{~g} \mathrm{~cm}^{-3}<\rho<10^{-7} \mathrm{~g} \mathrm{~cm}^{-3}$, the Helmholtz EOS and the EOS mentioned just above are smoothly blended. As for the latter EOS, the contribution to the pressure from the radiation is suppressed depending on the density and temperature in an optically thin regime (see Paper I). As in Papers I and II, energy deposition rates from the decay of ${ }^{56} \mathrm{Ni}$ and ${ }^{56} \mathrm{Co}$ are also implemented (see Paper I for details).

\subsection{Initial Conditions: Pre-supernova Models}

In this subsection, the pre-supernova models used as the initial conditions of the hydrodynamical simulations are described. Here, before the description, some properties of Sk $-69^{\circ} 202$ that are closely related to the study in this paper (the matter mixing) are briefly summarized as follows. The

\footnotetext{
8 Without the factor of $\sin \theta$ in $\phi^{\prime}=\phi \sin \theta$, the wavelengths of the perturbations around the polar axis become too small compared with those around the equatorial plane.
} 
Table 1

Properties of Pre-supernova Models

\begin{tabular}{lccccccccc}
\hline \hline Model & $M$ & $M_{\mathrm{C}+\mathrm{O}, \mathrm{c}}{ }^{\mathrm{a}}$ & $\begin{array}{c}M_{\mathrm{He}, \mathrm{c}}{ }^{\mathrm{b}} \\
\left(M_{\odot}\right)\end{array}$ & $\begin{array}{c}M_{\text {env }}{ }^{\mathrm{c}} \\
\left(M_{\odot}\right)\end{array}$ & $\begin{array}{c}R \\
(\mathrm{~cm})\end{array}$ & $\begin{array}{c}R \\
\left(R_{\odot}\right)\end{array}$ & $q \equiv M_{\mathrm{He}, \mathrm{c}} / M$ & Type $^{\mathrm{d}}$ & Evolution $^{\mathrm{e}}$ \\
\hline $\mathrm{b} 18.3$ & 18.3 & 2.87 & 3.98 & 14.3 & $2.12(12)^{\mathrm{f}}$ & 30.7 & 0.22 & BSG & Binary \\
$\mathrm{n} 16.3$ & 16.3 & 3.76 & 5.99 & 10.3 & $3.39(12)$ & 48.7 & 0.37 & BSG \\
\hline $\mathrm{s} 18.0^{\mathrm{g}}$ & 14.9 & 4.19 & 5.49 & 9.45 & $6.76(13)$ & 972 & 0.37 & RSG & Single \\
$\mathrm{s} 19.8^{\mathrm{g}}$ & 15.9 & 4.89 & 6.24 & 9.61 & $7.36(13)$ & 1058 & 0.39 & RSG & Single \\
\hline
\end{tabular}

Notes.

${ }^{\text {a }}$ Mass of the $\mathrm{C}+\mathrm{O}$ core.

${ }^{\mathrm{b}}$ Mass of the helium core.

${ }^{c}$ Mass of the hydrogen-rich envelope.

d Type of the pre-supernova model, i.e., "RSG" or "BSG."

e Evolution scenario, i.e., "binary" ("single") denotes a binary merger (single star) evolution.

${ }^{\mathrm{f}}$ Number in parentheses denotes the power of 10 .

${ }^{\mathrm{g}}$ The number in the name denotes the zero-age main-sequence mass.

luminosity and effective temperature of $\mathrm{Sk}-69^{\circ} 202$ are $(3-6) \times 10^{38} \mathrm{erg} \mathrm{s}^{-1}$ and $15,000-18,000 \mathrm{~K}$, respectively (Arnett et al. 1989b). Since, at the time of explosion, energy generation from hydrogen shell burning is generally negligible, the helium core mass is closely related to the luminosity; from the evolution models, it is in the range of $6 \pm 1 M_{\odot}$ for the case of the single-star evolution (Woosley 1988). With the ranges of the luminosity and effective temperature, the radius is estimated as (2-4) $\times 10^{12} \mathrm{~cm}$ (Arnett et al. 1989b).

In this paper, four pre-supernova models (denoted as n16.3, b18.3, s18.0, and s19.8) are adopted. Important properties of the models are summarized in Table 1 , where $M$ is the stellar mass, $M_{\mathrm{C}+\mathrm{O}, \mathrm{c}}$ is the $\mathrm{C}+\mathrm{O}$ core mass, $M_{\mathrm{He}, \mathrm{c}}$ is the helium core mass, $M_{\text {env }}$ is the hydrogen envelope mass, $R$ is the stellar radius (listed in units of $\mathrm{cm}$ and $R_{\odot}$ in the sixth and seventh columns, respectively), and $q \equiv M_{\mathrm{He}, \mathrm{c}} / M$ is the ratio of the helium core mass to the stellar mass. Here the values are all the ones at the time of the explosion. The ninth and 10th columns, "Type" and "Evolution," denote the types of the models, i.e., "BSG" or "RSG," and the evolution scenario, i.e., "single" star evolution or "binary" merger evolution. Models n16.3 and b18.3 are BSGs, whereas the other two, s18.0 and s19.8, are RSGs. As mentioned in Section 1, the progenitor of SN 1987A, $\mathrm{Sk}-69^{\circ} 202$, was a compact BSG at the time of the explosion, and the two RSG models are not appropriate for Sk $-69^{\circ} 202$ from the point of view of the effective temperature (stellar radius; see the sixth column in Table 1). The two models, however, are included to see the dependence on the progenitor models because the two RSG models have distinct properties compared with those of the BSG models.

Model b18.3 is a newly developed (Urushibata et al. 2018) compact BSG model based on the binary merger scenario (Podsiadlowski et al. 1990, 1992; Morris \& Podsiadlowski 2007). The overall binary merger scenario is as follows. A binary system with a large mass ratio consisting of a primary RSG $\left(\sim 15 M_{\odot}\right)$ and a secondary main-sequence star $\left(\sim 5 M_{\odot}\right)$ forms a common envelope through dynamical mass transfer from the primary to the secondary (here the masses of the two merging stars are taken from Morris \& Podsiadlowski 2007). ${ }^{9}$ The spiral-in of the core of the primary and secondary due to

\footnotetext{
9 In Podsiadlowski et al. (1990, 1992), the masses of the primary and secondary stars are 16 and $3 M_{\odot}$, respectively. In recent binary merger models for the progenitor of SN 1987A (Menon \& Heger 2017; Urushibata et al. 2018), the masses of two stars are in the range $14-17$ and 4-9 $M_{\odot}$, respectively.
}

the friction with the common envelope causes spin-up of the envelope and partial (aspherical) mass ejection from the envelope. Then, the secondary starts to transfer its mass to the core of the primary after the Roche lobe radius of the secondary becomes relatively smaller than its own stellar radius. During the mass transfer, part of the material from the secondary (composed of hydrogen-rich material) penetrates into the helium core of the primary, which triggers additional hydrogen burning and mixing of helium and CNO-processed material into the envelope. Eventually, the secondary is completely dissolved into the envelope of the primary to form a single rapidly rotating BSG. The properties of model b18.3 are listed in Table 1 in Urushibata et al. (2018; the model is labeled as "a" with a footnote); it is the outcome of the merger of two massive stars of 14 and $9.0 M_{\odot}$. This model satisfies all of the observational constrains of Sk $-69^{\circ} 202$, i.e., the final position in the H-R diagram (the observed luminosity and effective temperature), the red-to-blue transition about $20,000 \mathrm{yr}$ ago, the required surface abundances of helium and CNO-processed elements, and an ability to form a triple ring structure in the nebula. Hitherto, this model has not been investigated in the study of matter mixing.

Model n16.3 was obtained by combining an evolved $6 M_{\odot}$ He core corresponding to the zero-age main-sequence mass of $20 M_{\odot}$ (Nomoto \& Hashimoto 1988 ) with a $10.3 M_{\odot}$ hydrogen envelope. The hydrogen envelope was taken from an independent stellar evolution calculation (Saio et al. 1988b) in which an enhanced mass-loss rate and artificial mixing of helium-rich material into the hydrogen envelope were implemented to make a compact BSG that satisfies the observed luminosity and the effective temperature (Shigeyama \& Nomoto 1990). Model $\mathrm{n} 16.3$ has also been used in our previous studies on matter mixing (Papers I and II). It is noted that model $\mathrm{n} 16.3$ has been denoted as N20 in several studies (e.g., Wongwathanarat et al. 2015; Utrobin et al. 2019). In previous studies on matter mixing, this progenitor model has had difficulties reproducing the high-velocity ${ }^{56} \mathrm{Ni}$ of $\gtrsim 3000 \mathrm{~km} \mathrm{~s}^{-1}$ (e.g., Paper I; Wongwathanarat et al. 2015).

A distinct difference of the properties between the two BSG models, b18.3 and $\mathrm{n} 16.3$, is the ratios of core mass to envelope mass (see Table 1). As one can see, both the helium $\left(\sim 4 M_{\odot}\right)$ and $\mathrm{C}+\mathrm{O}\left(\sim 3 M_{\odot}\right)$ core masses of b18.3 are smaller than those of $n 16.3$ ( $\sim 6$ and $4 M_{\odot}$, respectively). On the contrary, the mass of the hydrogen envelope of b18.3 $\left(\sim 14 M_{\odot}\right)$ is larger 

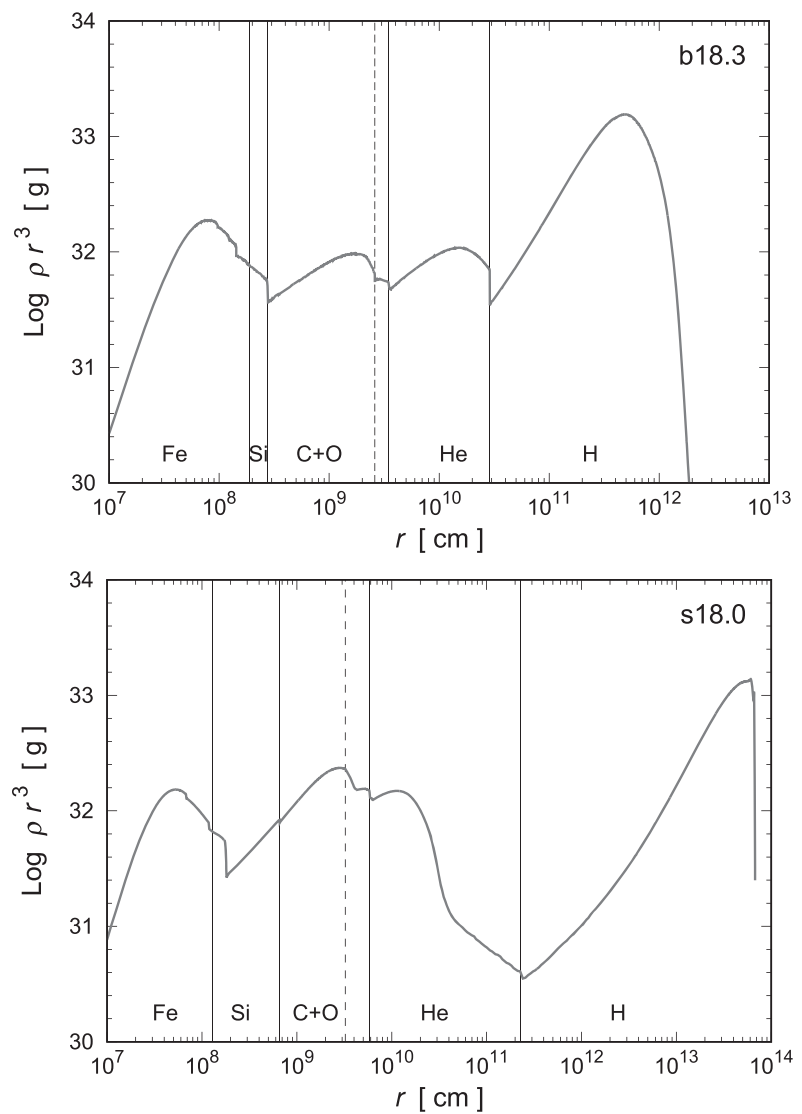
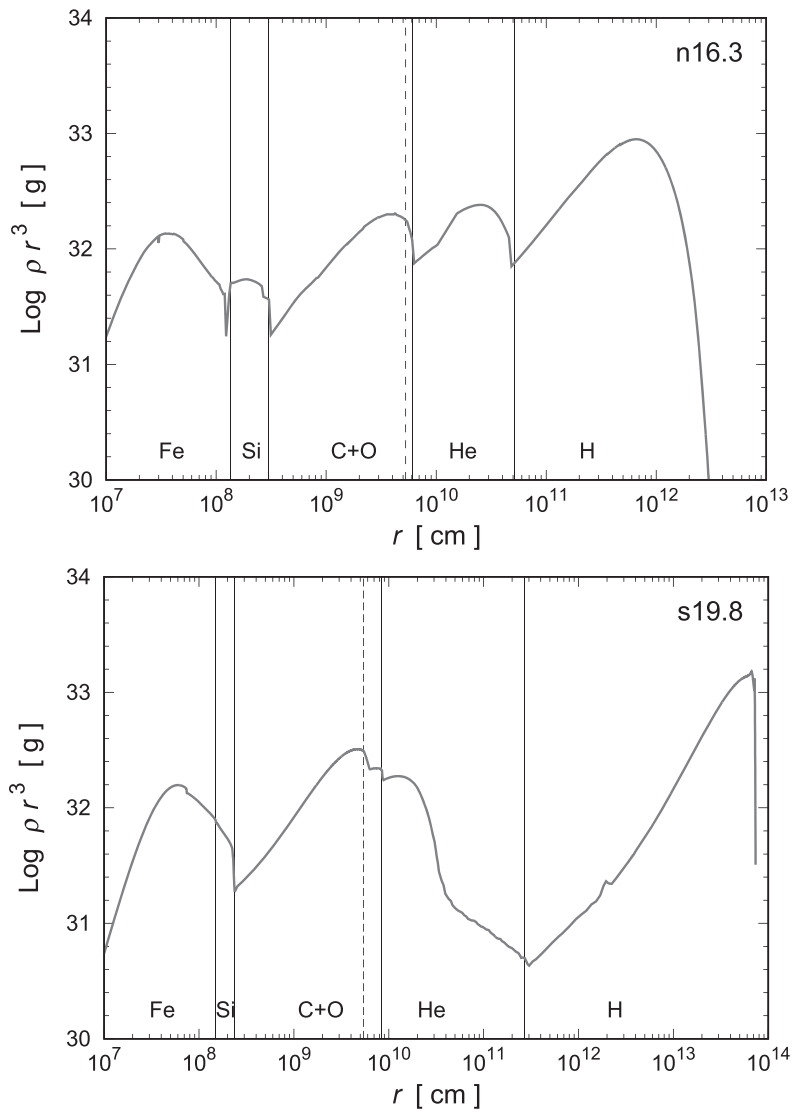

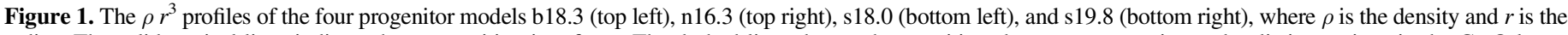

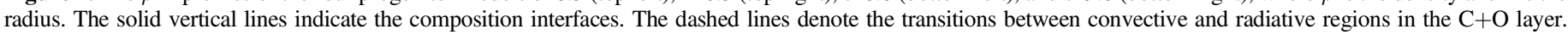

than that of $n 16.3\left(\sim 10 M_{\odot}\right)$. In other words, the ratio of the helium core mass to the stellar mass of b18.3 $(q=0.22)$ is smaller than that of $\mathrm{n} 16.3(q=0.37)$. The radius of $\mathrm{b} 18.3$ is also smaller than that of $n 16.3$ by a factor of about 0.6 .

Pre-supernova models s18.0 and s19.8 are taken from the supplementary data ${ }^{10}$ in Sukhbold et al. (2016). The number in each name does not denote the final stellar mass but rather the corresponding zero-age main-sequence mass, as in the paper. The two models are favored for SN 1987A in the point of view of the helium core mass $\left(\sim 6 M_{\odot}\right)$, but the radius (on the order of $\left.10^{13} \mathrm{~cm}\right)$ is very different from the observational constraints $\left(\sim 3 \times 10^{12} \mathrm{~cm}\right)$ by a factor of more than 10 . The two models are essentially the same as the corresponding models calculated in Woosley et al. (2002). Between the two RSG models, there are slight (but nonnegligible) differences in the properties. The ratios of the helium core mass to the stellar mass $(q \sim 0.4)$ have similar values as model $\mathrm{n} 16.3$, but the stellar radii are rather different from those of the BSG models.

In Figure 1, $\rho r^{3}$ profiles of the four models are shown, where $\rho$ is the density and $r$ is the radius. The top left, top right, bottom left, and bottom right panels are the profiles of b18.3, n16.3, s18.0, and s19.8, respectively. The solid vertical lines indicate the composition interfaces. The dashed vertical lines denote the transition between convective and radiative regions in the $\mathrm{C}+\mathrm{O}$ layer. The gradient of $\rho r^{3}$ provides useful information on where and when the supernova shock wave is

\footnotetext{
${ }^{10}$ The supplementary data are taken from Sukhbold et al. (2016).
}

accelerated or decelerated. In the self-similar solution of a point explosion in a power-law density profile of $\rho(r) \propto r^{-\omega}$ (Sedov 1959), the velocity of the blast wave can be expressed as $v_{\mathrm{sh}}(t) \propto t^{(\omega-3) /(5-\omega)}$. From the relation, one finds that if the power of the density profile $-\omega$ is -3 , the velocity of the blast wave is constant. Equivalently, if the gradient of $\rho r^{3}$ is positive (corresponding to the case of $\omega<3$ ), the velocity of the blast wave decreases (the blast wave is decelerated) at the position, and vice versa. In this way, the density structure affects how the supernova shock wave propagates in the presupernova star. Since the radial velocity of the supernova ejecta is very roughly proportional to the radius, basically, it is difficult for the inner ejecta to catch up with the highervelocity outer ejecta. But, depending on the complicated density structure, as seen in Figure 1, the propagation of the blast wave and the expansion of the inner ejecta could drastically change among the progenitor models. Additionally, the condition of the RT instability is $\nabla P \cdot \nabla \rho<0$ (Chevalier 1976), and the structure of the density gradient is also important for the growth of the RT instability. For the comparison among the models, the $\rho r^{3}$ profiles are shown on a single plot (Figure 2). As one can see, among the models, there are large differences in the structures of the $\mathrm{C}+\mathrm{O}$ layer, helium layer, and hydrogen envelope. The binary merger model, b18.3, has the flattest $\rho r^{3}$ gradient in the $\mathrm{C}+\mathrm{O}$ layer. The structures of the RSG models in the helium layer and hydrogen envelope are similar between the two RSG models. In the RSG models, the blast wave overall accelerates inside the helium layer, but the situation is opposite in the hydrogen 


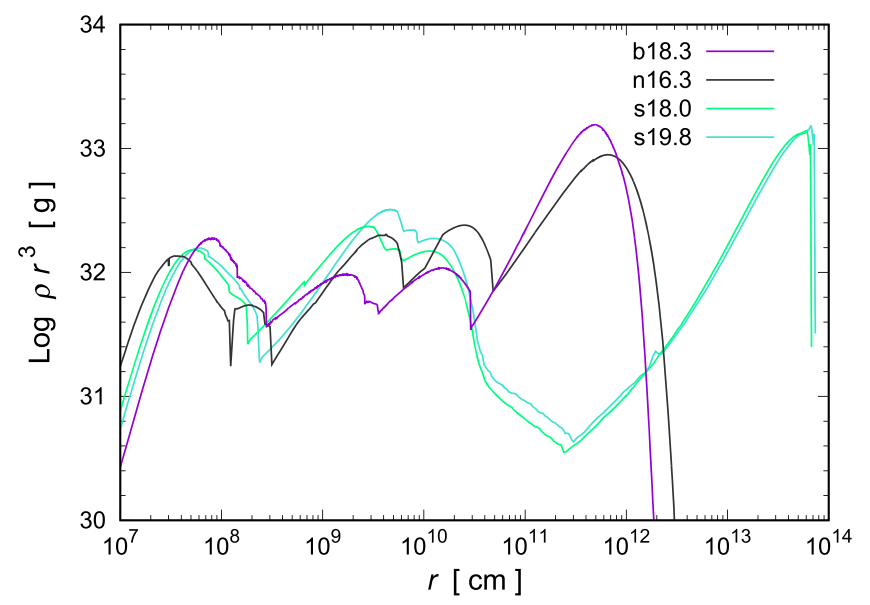

Figure 2. Same as Figure 1, but the four $\rho r^{3}$ profiles are plotted on the same figure.

envelope. On the other hand, the $\rho r^{3}$ gradient of the BSG models in the helium layer is overall positive, except for a thin region at the outer layer, and the structures in the hydrogen envelope are rather different from those of the RSG models because of the large differences in the radius.

\section{Simulation Models}

In this paper, we perform 1D and 3D hydrodynamical simulations. Here, the hydrodynamical models and related model parameters are described in detail.

\subsection{Models of $1 D$ Simulations}

In order to assess the dependence of the matter mixing on the progenitor models, we first perform 1D hydrodynamical simulations. As in previous papers (e.g., Ebisuzaki et al. 1989; Benz \& Thielemann 1990; Mueller et al. 1991), including Paper I, from spherical 1D hydrodynamical simulations of the blast-wave propagation in an expanding star, stability analyses of instabilities for the progenitor models can be done. In order to evaluate the time-integrated growths of instabilities (growth factors), several 1D simulations are performed for each progenitor model changing the initial injection energy, $E_{\text {in. }}$. For the simulations, the same numerical code, FLASH, is used, and the basic method is the same as described in Section 2.1. But the adopted coordinate system is the spherical coordinate here, and the resolution of the simulations and treatments of inner regions corresponding to the compact object are different because of the differences between the two coordinate systems. For the treatments in the spherical coordinate, see Paper I. For 1D simulations, the model parameter on explosions is only $E_{\mathrm{in}}$. From the observations of the optical light curves and theoretical modeling of the light curves (e.g., Woosley 1988; Shigeyama \& Nomoto 1990), the explosion energy of SN 1987A has been deduced. For example, the range of the explosion energy, $E_{\text {exp }}$, was estimated as $E_{\exp } / M_{\text {env }}=$ $(1.1 \pm 0.3) \times 10^{50} \mathrm{erg} M_{\odot}^{-1}$ in Shigeyama \& Nomoto (1990). The explosion energy depends on the hydrogen envelope mass in this case. By substituting the value of the envelope mass of the binary merger model b18.3 $\left(M_{\mathrm{env}}=14.3 M_{\odot}\right)$ into the equation, $E_{\text {exp }} / M_{\text {env }}=(1.1 \pm 0.3) \times 10^{50} \mathrm{erg} M_{\odot}^{-1}$, one can obtain the explosion energy as $E_{\text {exp }}=(1.1-2.0) \times 10^{51} \mathrm{erg}$. The deduced values of the explosion energy have not converged among the studies, but overall, the values are within the range of
$E_{\text {exp }}=(0.8-2.0) \times 10^{51}$ erg. ${ }^{11}$ From the results in Papers I and II, it has been empirically found that the final explosion energy is roughly approximated as $E_{\text {exp }} \simeq\left(E_{\text {in }}-0.5 \times 10^{51} \mathrm{erg}\right)$. Then, as the values of the parameter, $E_{\mathrm{in}}$, the four values, $(1.5,2.0$, 2.5 , and 3.0$) \times 10^{51} \mathrm{erg}$, are adopted in this paper. The last value is outside the range above, but we include it as an extreme case.

Here we briefly review the method of the stability analysis. For the analysis, two kind of instabilities are considered. One is the RT instability for an incompressible fluid, and the other is an instability for a compressible fluid (convection). The condition of the RT instability (Chevalier 1976) is expressed as

$$
\frac{\mathcal{R}}{\mathcal{P}}<0,
$$

where $\mathcal{R} \equiv \partial \ln \rho / \partial r$ and $\mathcal{P} \equiv \partial \ln P / \partial r$ are the reciprocals of the density and pressure scale heights, respectively. Here $\rho$ is the density, $r$ is the radius, and $P$ is the pressure. The criterion of the convective instability for a compressible fluid (Schwarzschild criterion; e.g., Bandiera 1984) is

$$
\frac{\mathcal{R}}{\mathcal{P}}<\frac{1}{\gamma},
$$

where $\gamma$ is the adiabatic index. The growth rate of the RT instability is written as

$$
\sigma_{\mathrm{i}}=\sqrt{-\frac{P}{\rho} \mathcal{P} \mathcal{R}} .
$$

The growth rate of the convective instability is

$$
\sigma_{\mathrm{c}}=\frac{c_{\mathrm{s}}}{\gamma} \sqrt{\mathcal{P}^{2}-\gamma \mathcal{P} \mathcal{R}}
$$

where $c_{\mathrm{s}}$ is the sound speed. From the growth rate, the timeintegrated growth (growth factor) for each instability is calculated as

$$
\left.\frac{\zeta}{\zeta_{0}}\right|_{t}=\exp \left(\int_{0}^{t} \operatorname{Re}[\sigma] d t^{\prime}\right),
$$

where $\zeta_{0}$ is the initial amplitude of a perturbation, $\zeta$ is the amplitude of the perturbation at time $t$, and $\sigma=\sigma_{\mathrm{i}}\left(\sigma=\sigma_{\mathrm{c}}\right)$ for an incompressible (a compressible) fluid. Based on the results of the 1D simulations described in Section 3.1, the growth factors are deduced at each mass coordinate at time $t$. The growth factors are based on a local linear analysis of instabilities. Then, once the instabilities enter a nonlinear regime, the growth rates are no longer followed by Equations (3) and (4) in a realistic multidimensional situation. Actually, the growth rate of the RT instability is proportional to the square root of the wavenumber of the perturbation (e.g., Ebisuzaki et al. 1989), and after the nonlinear regime, merging of fingers (inverse cascading) may occur to form larger-scale structures (Hachisu et al. 1992). Therefore, the values of the growth factors should not be taken quantitatively but only qualitatively. Nevertheless, the growth rates can be useful to grasp where instabilities are easy to grow and the dependence on the progenitor models.

\footnotetext{
${ }^{11}$ The range of the explosion energy was summarized in Table 1 in Handy et al. (2014).
} 
Table 2

Models of 3D Simulations and Parameters

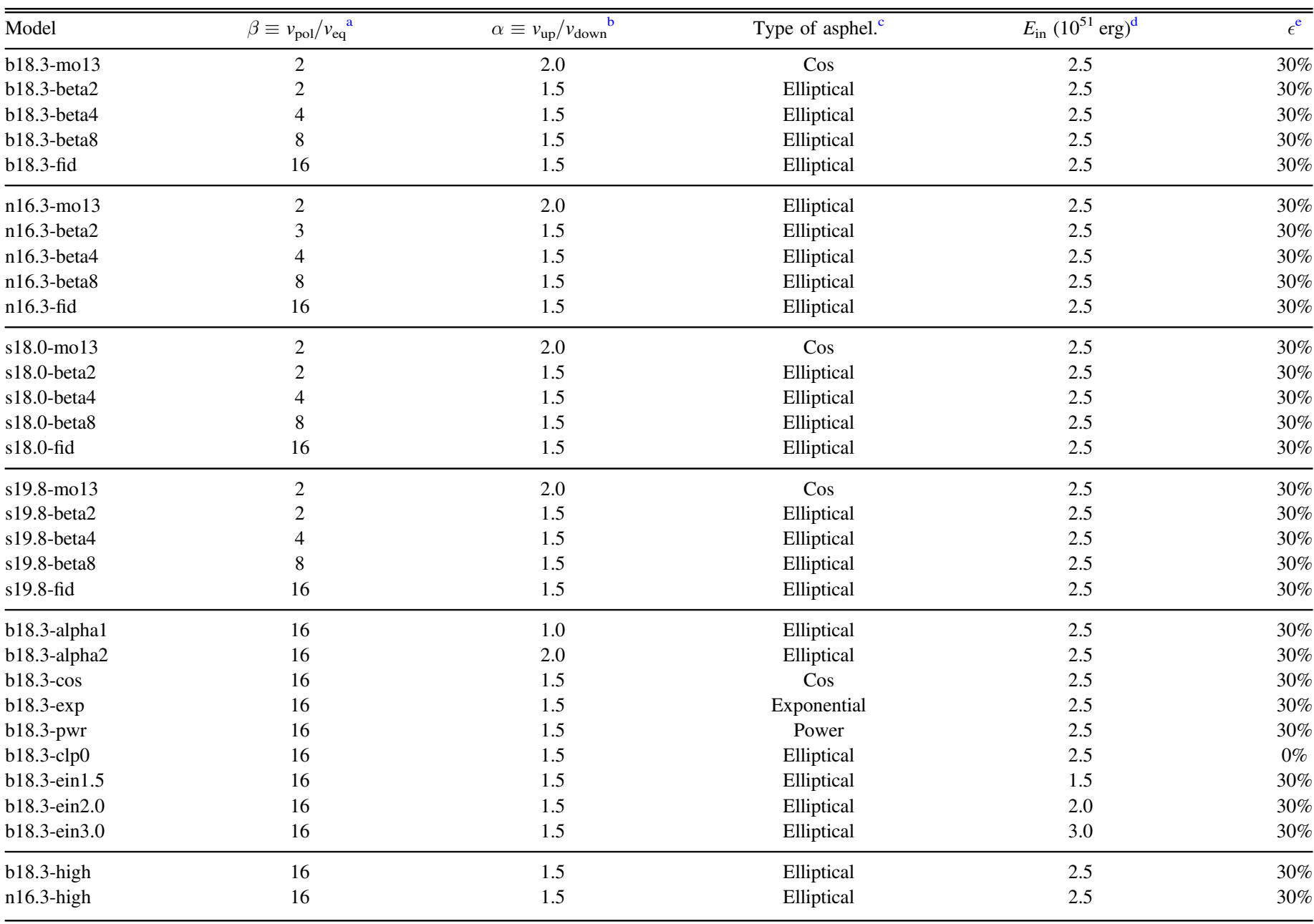

Notes.

${ }^{\text {a }}$ Ratio of the initial radial velocities along the polar to the equatorial ( $x-y$ plane) directions.

${ }^{\mathrm{b}}$ Ratio of the initial radial velocities along the positive to the negative $z$-directions.

${ }^{c}$ Type of the distribution of the initial radial velocities described in Appendix A, i.e., "cos," "exponential," "power," or "elliptical," which correspond to the shapes of the functions, $f(\theta)$, in Equations (8)-(11), respectively.

${ }^{d}$ Energy initially injected to initiate the explosion.

${ }^{\mathrm{e}}$ Amplitude of the fluctuations in the initial radial velocities.

\subsection{Models of 3D Simulations}

As noted in Section 1, we first perform 3D lower-resolution simulations to explore a wide range of parameters related to aspherical explosions and the progenitor models. Among the models with the best parameter set of the explosion asphericity, two 3D high-resolution simulations are performed for the two BSG models, i.e., b18.3 and n16.3. For the parameters related to the aspherical explosions, see Appendices A and B. The models of the $3 \mathrm{D}$ simulations and the adopted values of the related parameters are summarized in Table 2, where $\beta \equiv v_{\mathrm{pol}} / v_{\mathrm{eq}}$ is the ratio of the initial radial velocities along the polar to the equatorial ( $x-y$ plane $)$ directions, and $\alpha \equiv v_{\text {up }} / v_{\text {down }}$ is the ratio of the initial radial velocities along the positive to the negative $z$-directions. The fourth column denotes the type of distribution of the initial radial velocities described in Appendix A, i.e., "cos," "exponential," "power," or "elliptical," which correspond to the shapes of the functions, $f(\theta)$, in Equations (8)-(11), respectively. The sixth column, $\epsilon$, indicates the amplitude of the fluctuations in the initial radial velocities. The angular dependence of the fluctuations is described in Appendix B.

The nomenclature of the models is as follows. For example, in the case of "b18.3-mo13," the part before the hyphen, "b18.3," denotes the adopted pre-supernova model, and the latter part, "mo13," indicates the properties of the adopted parameter set related to the initial asphericity of the explosion and the injected energy. The models with "mo13" adopt the parameter set corresponding to the ones in the best model, AM2, in Paper I. The models with "fid" adopt the fiducial (the best) parameter set in this paper. The models with "beta2," "beta4," and "beta8" have the values of the parameter $\beta \equiv v_{\mathrm{pol}} / v_{\mathrm{eq}}$ as 2,4 , and 8 , respectively. In those models, only the values of $\beta$ are different from the parameter set adopted in the models with "fid." In a similar way, the models with "alpha1" and "alpha2" have the values of the parameter $\alpha \equiv v_{\text {up }} / v_{\text {down }}$ set to be 1.0 and 2.0 , respectively. The models with "cos," "exp," and "pwr" adopt the types of the initial 
asphericity of the explosion as "cos," "exponential," and "power," respectively. In the model with "clp0," the value of the parameter $\epsilon$ is $0 \%$. The models with "ein 1.5," "ein2.0," and "ein 3.0" have the values of the parameter $E_{\text {in }}$ as $(1.5,2.0$, and $3.0) \times 10^{51} \mathrm{erg}$, respectively. Finally, the models with "high" have the same parameter sets as in the corresponding models with "fid," but the simulations are performed with the highest resolution in this paper.

In Paper I, we explored mildly aspherical explosions with the progenitor model of $\mathrm{n} 16.3$. The obtained maximum velocity of ${ }^{56} \mathrm{Ni}$, however, is at most only $\sim 3000 \mathrm{~km} \mathrm{~s}^{-1}$, and the tails $\left(\sim 4000 \mathrm{~km} \mathrm{~s}^{-1}\right)$ of the observed [Fe II] line profiles were not explained in Paper I. It is noted that the corresponding model to the best model in Paper I, AM2, is model n16.3-mo13 in this paper (see Table 2). Then, in this paper, we explore a wider range for the asphericity of the explosions. For example, as can be seen in Figure 26 in Appendix A, in the best model in Paper I, the angle dependence of the initial radial velocities is similar to the distribution for $\beta=2$, shown in the top left panel. In this paper, explosions in which higher initial radial velocities are more concentrated around the polar axis (see the distribution for $\beta=16$ in the bottom right panel) are also considered. As for the types of the initial asphericity of the explosion, the "elliptical" form is adopted as a fiducial form because we found that models with the "elliptical" form overall better reproduce the observational requirements for SN 1987A discussed in Section 4.2. The impacts of the types can be investigated by comparing the results among the models b18.3fid, b18.3-cos, b18.3-exp, and b18.3-pwr, among which only the types of the initial asphericity of the explosion are different (see Section 4.2). Moreover, in order to investigate the impact of the progenitor model dependence, the four progenitor models (the two BSG models and the other two RSG models) are included.

\section{Results}

In this section, the results of the 1D simulations (Section 4.1), 3D simulations with lower resolution (Section 4.2), and 3D highresolution simulations (Section 4.3) are presented in sequence.

\subsection{Results of $1 D$ Simulations}

In this subsection, the results of stability analyses of the four pre-supernova models are shown. The growth factors at the time when the shock wave reaches the radius of about $5 \times 10^{14} \mathrm{~cm}$ (after the shock breakout for all cases) are shown in Figure 3. From top to bottom, progenitor models b18.3, $\mathrm{n} 16.3$, s18.0, and s19.8 are shown. From left to right, the cases of the injected energies $E_{\mathrm{in}}=(1.5,2.0,2.5$, and 3.0) $\times$ $10^{51} \mathrm{erg}$ are depicted. Red solid lines are the growth factors for a compressible fluid, and black dashed lines are those for an incompressible fluid. Thin vertical lines are the composition interfaces. As one can see, growth factors are salient around the composition interfaces of $\mathrm{He} / \mathrm{H}$ and/or $\mathrm{C}+\mathrm{O} / \mathrm{He}$, as shown in previous studies (e.g., Ebisuzaki et al. 1989; Benz \& Thielemann 1990; Mueller et al. 1991). Since the condition for the RT instability for an incompressible fluid is always more stringent than that for the convective instability (Schwarzschild criterion) for a compressible fluid, the development of the growth factors for the convective instability dominates the one for the RT instability. The top two rows are for BSG models, and the bottom two rows are for RSG models.
In BSG models, growth factors are high around both the $\mathrm{C}+\mathrm{O} /$ $\mathrm{He}$ and $\mathrm{He} / \mathrm{H}$ interfaces. On the other hand, in RSG models, growth factors are outstanding only around the $\mathrm{He} / \mathrm{H}$ interfaces, which is attributed to the fact that the gradients of the $\rho r^{3}$ value are overall negative in the helium layer of the two RSG models, in contrast to the case of the BSG models (see Figure 1). Focusing on the binary merger model (b18.3), the growth factors seem to be proportional to the injected energies $E_{\text {in }}$, in particular at the $\mathrm{He} / \mathrm{H}$ composition interface. The growth factors depend on several factors, e.g., where and when the conditions, Equations (1) and (2), are realized; the steepness of the density and pressure gradients; and the time for instabilities to grow. Then, the situation could change depending on the progenitor models and explosion energies. For the cases of the binary merger model (b18.3), a more energetic explosion probably makes the pressure gradients steeper than those for less energetic models. As for the other BSG model (n16.3), the growth factors are not sensitive to the explosion energies. For the case of model s18.0 (RSG), in the less energetic model (left panel), the growth factors are most prominent around the $\mathrm{He} / \mathrm{H}$ composition interface.

\subsection{Results of 3D Simulations: Lower-resolution Cases}

In this section, the results of the $3 \mathrm{D}$ simulations with lower resolution are presented. The results are summarized in Table 3, where $E_{\text {exp }}$ is the explosion energy (see Equation (6) for the definition); $M_{\mathrm{ej}}\left({ }^{56} \mathrm{Ni}\right)$ is the ejected total mass of ${ }^{56} \mathrm{Ni}$; $M_{\text {ej }}\left({ }^{44} \mathrm{Ti}\right)$ is the ejected total mass of ${ }^{44} \mathrm{Ti} ; M_{3.0}\left({ }^{56} \mathrm{Ni}\right), M_{4.0}$ $\left({ }^{56} \mathrm{Ni}\right)$, and $M_{4.7}\left({ }^{56} \mathrm{Ni}\right)$ are the masses of ${ }^{56} \mathrm{Ni}$ for which the radial velocity is $\geqslant 3000, \geqslant 4000$, and $\geqslant 4700 \mathrm{~km} \mathrm{~s}^{-1}$, respectively; the value in the eighth column, $M_{3.0} / M_{\mathrm{ej}}\left({ }^{56} \mathrm{Ni}\right)$, is the ratio of the values of the fifth to third columns; and $v_{\mathrm{NS}}$ is the NS kick velocity (see Section 5.3 and Equation (7)). The 10th column, "No.," denotes the sequential serial number (model number) for Figure 11. The values in Table 3 are obtained at the end of the simulation when the blast wave reaches $\sim 2 \times 10^{14} \mathrm{~cm}$ (after the shock breakout for all models). Explosion energies are defined by the expression

$$
E_{\exp }=\iiint_{V}\left(\frac{1}{2} \rho v^{2}+\rho E+\rho \Phi\right) d x d y d z
$$

where $V$ is the computational domain, $v$ is the velocity, $E$ is the internal energy, and $\Phi$ is the gravitational potential.

As noted in Section 3.1, the explosion energy of SN 1987A has been estimated from the observations in the range of (1-2) $\times$ $10^{51} \mathrm{erg}$. The injected energy $E_{\text {in }}$ is $2.5 \times 10^{51} \mathrm{erg}$, except for models b18.3-ein1.5, b18.3-ein2.0, and b18.3-ein3.0, in which $E_{\text {in }}=(1.5,2.0$, and 3.0$) \times 10^{51} \mathrm{erg}$, respectively. From the second column of Table 3 , obtained explosion energies, $E_{\text {exp }}$, from the lower-resolution simulations are roughly $\lesssim 2 \times 10^{51} \mathrm{erg}$ for the models with $E_{\text {in }}=2.5 \times 10^{51} \mathrm{erg}$, and those values are within the accepted range, i.e., (1-2) $\times 10^{51} \mathrm{erg}$, mentioned above. For models b18.3-ein1.5, b18.3-ein2.0, and b18.3-ein3.0, the obtained $E_{\text {exp }}$ is roughly $(1,1.5$, and 2.5$) \times 10^{51} \mathrm{erg}$, respectively. As one can see, the $E_{\exp }$ values are well approximated as $E_{\text {in }}-0.5$ $\times 10^{51} \mathrm{erg}$. The $E_{\text {exp }}$ value for model b18.3-ein3.0 is outside the accepted range. Then, model b18.3-ein3.0 is an extreme case.

From the theoretical modeling of the observed optical light curves (e.g., Woosley 1988; Shigeyama \& Nomoto 1990), the mass of ejected ${ }^{56} \mathrm{Ni}$ has been deduced as $0.07 M_{\odot}$. Obtained ejected masses of ${ }^{56} \mathrm{Ni}$ from the simulations depend on the 


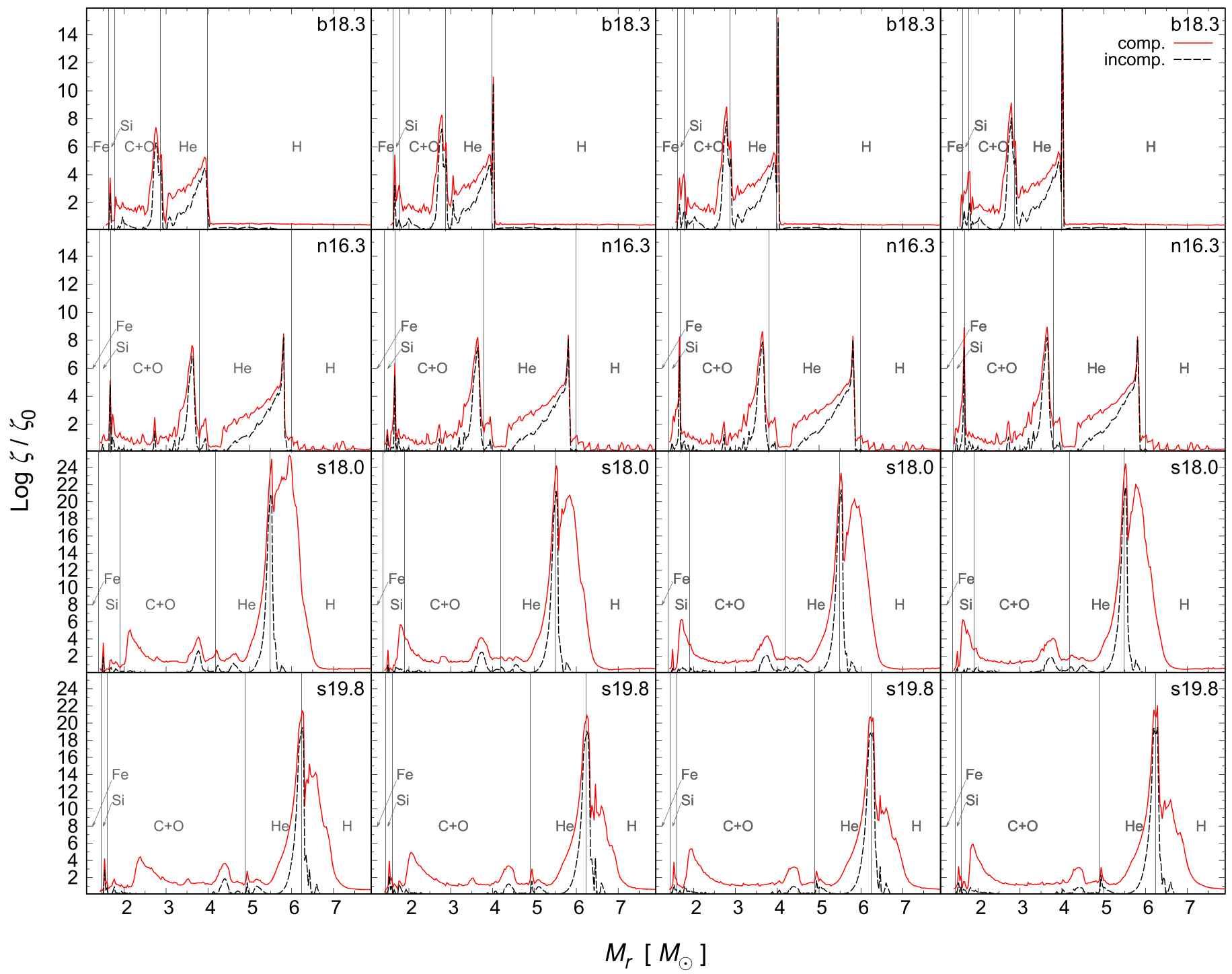

Figure 3. Growth factors, $\zeta / \zeta_{0}$, as a function of the mass coordinate, $M_{r}$, for the progenitor models b18.3 (first row), n16.3 (second row), s18.0 (third row), and s19.8 (fourth row) at the time when the shock wave reaches the radius of about $5 \times 10^{14} \mathrm{~cm}$. From left to right, the cases of the injected energies $E_{\text {in }}=(1.5,2.0,2.5$, and 3.0) $\times 10^{51}$ erg are shown, respectively. The red solid lines are the growth factors for compressible fluid, and the black dashed lines are those for incompressible fluid. Thin vertical lines are the composition interfaces. See the text for details.

degrees of the asymmetry of bipolar-like explosion $\left(\beta \equiv v_{\mathrm{pol}} / v_{\mathrm{eq}}\right)$, asymmetries against the equatorial plane $\left(\alpha \equiv v_{\text {up }} / v_{\text {down }}\right)$, and progenitor models. For example, looking at the values for models b18.3-beta2, b18.3-beta4, b18.3-beta8, and b18.3-fid ( $\beta=2,4,8$, and 16 , respectively), the larger the $\beta$ value, the smaller the ejected mass of ${ }^{56} \mathrm{Ni}$. Comparing models b18.3-alpha1, b18.3-fid, and b18.3-alpha2 $(\alpha=1.0$, 1.5 , and 2.0, respectively), the larger the $\alpha$ value, the smaller the ejected mass of ${ }^{56} \mathrm{Ni}$. In the order s19.8-fid, b18.3-fid, n16.3-fid, and s18.0-fid, the mass of ejected ${ }^{56} \mathrm{Ni}$ is increasing. The dependence of the mass of ejected ${ }^{56} \mathrm{Ni}$ on the presupernova models may reflect the structure (density and temperature) of the innermost regions around the composition interface of $\mathrm{Fe} / \mathrm{Si}$ (see Figure 1). Overall, the obtained values of the mass of ejected ${ }^{56} \mathrm{Ni}$ are $(0.8-1) \times 10^{-1} M_{\odot}$, but for some models, e.g., s18.0-mo13 and s18.0-beta2, the ejected mass of ${ }^{56} \mathrm{Ni}\left(\sim 0.13 M_{\odot}\right)$ is a bit large.

As mentioned in Section 1, the mass of ${ }^{44} \mathrm{Ti}$ has been estimated as $(3.1 \pm 0.8) \times 10^{-4} M_{\odot}$ (Grebenev et al. 2012) or $(1.5 \pm$ $0.3) \times 10^{-4} M_{\odot}$ (Boggs et al. 2015) from the observations of direct $\gamma$-ray lines from the decay of ${ }^{44} \mathrm{Ti}$. Overall, the obtained mass of ejected ${ }^{44} \mathrm{Ti}$ is within the orders of $10^{-4}-10^{-3} M_{\odot}$. In general, the amount of ${ }^{44} \mathrm{Ti}$ synthesized by spherical core-collapse supernovae or neutrino-driven supernova explosions are of the order of $10^{-5} M_{\odot}$ (Rauscher et al. 2002; Fujimoto et al. 2011), and the large values $\left(\sim 10^{-4} M_{\odot}\right)$ deduced from the observations are in some sense a mystery. A jetlike (globally aspherical) explosion could be essential for a strong alpha-rich freeze-out to be realized to obtain a high mass ratio of ${ }^{44} \mathrm{Ti}$ to ${ }^{56} \mathrm{Ni}$ (Nagataki et al. 1997, 1998a).

It is noted that the calculations of the explosive nucleosynthesis in this paper are performed with only the small nuclear reaction network (19 nuclei are included). Then, the amount of ${ }^{44} \mathrm{Ti}$ (roughly 2 orders of magnitude less than that of ${ }^{56} \mathrm{Ni}$ ) is inaccurate compared with the value of ${ }^{56} \mathrm{Ni}$. Additionally, the innermost regions around the composition interface of $\mathrm{Fe} / \mathrm{Si}$ where the explosive nucleosynthesis occurs are slightly neutronrich. Then, the synthesis of neutron-rich isotopes, ${ }^{57} \mathrm{Ni}$ and ${ }^{58} \mathrm{Ni}$, is also expected. As demonstrated in the Appendix in Paper II, the calculated masses of ${ }^{56} \mathrm{Ni}$ and ${ }^{44} \mathrm{Ti}$ could be overestimated by 
Table 3

Results of 3D Simulation Models

\begin{tabular}{|c|c|c|c|c|c|c|c|c|c|}
\hline Model & $\begin{array}{l}E_{\exp }^{\mathrm{a}} \\
\text { (erg) }\end{array}$ & $\begin{array}{c}M_{\mathrm{ej}}\left({ }^{56} \mathrm{Ni}\right)^{\mathrm{b}} \\
\left(M_{\odot}\right)\end{array}$ & $\begin{array}{c}M_{\mathrm{ej}}\left({ }^{44} \mathrm{Ti}\right)^{\mathrm{c}} \\
\left(M_{\odot}\right)\end{array}$ & $\begin{array}{c}M_{3.0}\left({ }^{56} \mathrm{Ni}\right)^{\mathrm{d}} \\
\left(M_{\odot}\right)\end{array}$ & $\begin{array}{c}M_{4.0}\left({ }^{56} \mathrm{Ni}\right)^{\mathrm{e}} \\
\left(M_{\odot}\right)\end{array}$ & $\begin{array}{c}M_{4.7}\left({ }^{56} \mathrm{Ni}\right)^{\mathrm{f}} \\
\left(M_{\odot}\right)\end{array}$ & $\begin{array}{c}M_{3.0} / M_{\mathrm{ej}}\left({ }^{56} \mathrm{Ni}\right)^{\mathrm{g}} \\
(\cdots)\end{array}$ & $\begin{array}{c}v_{\mathrm{NS}}{ }^{\mathrm{h}} \\
\left(\mathrm{km} \mathrm{s}^{-1}\right)\end{array}$ & No. ${ }^{i}$ \\
\hline b18.3-mo13 & $1.95(51)^{\mathrm{j}}$ & $9.83(-2)^{\mathrm{k}}$ & $7.79(-4)^{\mathrm{k}}$ & $9.02(-4)$ & $1.02(-5)$ & $1.69(-7)$ & $9.17(-3)^{k}$ & $5.05(2)$ & 1 \\
\hline b18.3-beta2 & $1.95(51)$ & $1.06(-1)$ & $7.76(-4)$ & $1.51(-4)$ & $9.75(-7)$ & $8.86(-9)$ & $1.43(-3)$ & $3.35(2)$ & 2 \\
\hline b18.3-beta8 & $1.97(51)$ & $8.39(-2)$ & $7.12(-4)$ & $8.12(-3)$ & $1.43(-3)$ & $3.59(-4)$ & $9.68(-2)$ & $2.83(2)$ & 4 \\
\hline b18.3-fid & $1.99(51)$ & $8.10(-2)$ & $7.39(-4)$ & $1.12(-2)$ & $1.89(-3)$ & $2.81(-4)$ & $1.38(-1)$ & $2.75(2)$ & 5 \\
\hline n16.3-beta4 & $1.89(51)$ & $1.01(-1)$ & $6.04(-4)$ & $1.35(-3)$ & $5.55(-6)$ & $4.32(-8)$ & $1.33(-2)$ & $3.43(2)$ & 8 \\
\hline n16.3-beta8 & $1.90(51)$ & $9.53(-2)$ & $6.58(-4)$ & $4.87(-3)$ & $8.07(-4)$ & $9.03(-5)$ & $5.11(-2)$ & $3.34(2)$ & 9 \\
\hline n16.3-fid & $1.91(51)$ & $9.04(-2)$ & $7.17(-4)$ & $1.27(-3)$ & $6.08(-5)$ & $1.78(-6)$ & $1.40(-2)$ & $3.20(2)$ & 10 \\
\hline $\mathrm{s} 18.0-\mathrm{mo} 13$ & $1.85(51)$ & $1.36(-1)$ & $1.15(-3)$ & $6.74(-4)$ & $7.12(-5)$ & $2.24(-5)$ & $4.95(-3)$ & $5.67(2)$ & 11 \\
\hline s18.0-beta2 & $1.87(51)$ & $1.33(-1)$ & $1.03(-3)$ & $2.60(-4)$ & $1.30(-5)$ & $1.62(-6)$ & $1.95(-3)$ & 3.99 (2) & 12 \\
\hline s18.0-beta4 & $1.85(51)$ & $1.27(-1)$ & $1.11(-3)$ & $9.19(-4)$ & $2.01(-4)$ & $6.76(-5)$ & $7.21(-3)$ & $3.28(2)$ & 13 \\
\hline s19.8-beta2 & $1.89(51)$ & $8.96(-2)$ & $8.47(-4)$ & $1.10(-4)$ & $4.18(-6)$ & $3.20(-7)$ & $1.23(-3)$ & $3.89(2)$ & 17 \\
\hline s19.8-beta4 & $1.88(51)$ & $8.83(-2)$ & $7.61(-4)$ & $4.29(-3)$ & $1.36(-3)$ & $4.90(-4)$ & $4.86(-2)$ & $3.51(2)$ & 18 \\
\hline s19.8-beta8 & $1.88(51)$ & $8.36(-2)$ & $8.25(-4)$ & $6.95(-3)$ & $2.73(-3)$ & $1.13(-3)$ & $8.31(-2)$ & $3.31(2)$ & 19 \\
\hline s19.8-fid & $1.89(51)$ & $7.80(-2)$ & $8.93(-4)$ & $6.93(-3)$ & $2.40(-3)$ & $8.87(-4)$ & $8.88(-2)$ & 2.99 (2) & 20 \\
\hline b18.3-alpha1 & $1.98(51)$ & $8.09(-2)$ & $6.61(-4)$ & $3.17(-3)$ & $2.79(-4)$ & $4.03(-5)$ & $3.92(-2)$ & $2.13(0)$ & 21 \\
\hline b18.3-alpha2 & $1.99(51)$ & $7.45(-2)$ & $7.70(-4)$ & $1.47(-2)$ & $4.55(-3)$ & $1.22(-3)$ & $1.98(-1)$ & $4.23(2)$ & 22 \\
\hline b18.3-cos & $2.00(51)$ & $8.53(-2)$ & $8.61(-4)$ & $2.59(-3)$ & $6.00(-5)$ & $8.30(-7)$ & $3.03(-2)$ & $2.59(2)$ & 23 \\
\hline b18.3-exp & $2.00(51)$ & $7.57(-2)$ & $7.71(-4)$ & $4.94(-3)$ & $3.32(-4)$ & $7.53(-6)$ & $6.53(-2)$ & $2.62(2)$ & 24 \\
\hline b18.3-pwr & $2.00(51)$ & $7.91(-2)$ & $7.75(-4)$ & $4.24(-3)$ & $2.85(-4)$ & $8.08(-6)$ & $5.35(-2)$ & $2.62(2)$ & 25 \\
\hline b18.3-clp0 & $1.99(51)$ & $8.07(-2)$ & $7.39(-4)$ & $1.32(-2)$ & $1.89(-3)$ & $4.36(-4)$ & $1.64(-1)$ & $2.78(2)$ & 26 \\
\hline b18.3-ein1.5 & $9.87(50)$ & $4.15(-2)$ & $3.53(-4)$ & $3.83(-5)$ & $2.51(-8)$ & $3.53(-11)$ & $9.25(-4)$ & 1.59 (2) & 27 \\
\hline b18.3-ein2.0 & $1.49(51)$ & $6.45(-2)$ & $5.89(-4)$ & $2.82(-3)$ & $1.85(-4)$ & $1.94(-5)$ & $4.38(-2)$ & $2.42(2)$ & 28 \\
\hline
\end{tabular}

Notes.

${ }^{a}$ Explosion energy estimated by Equation (6) at the end of the simulation.

${ }^{b}$ Mass of total ejected ${ }^{56} \mathrm{Ni}$ that has a positive radial velocity at the end of the simulation.

${ }^{\mathrm{c}}$ Mass of total ejected ${ }^{44} \mathrm{Ti}$ that has a positive radial velocity at the end of the simulation.

${ }^{\mathrm{d}}$ Mass of ${ }^{56} \mathrm{Ni}$ that has a velocity higher than $3000 \mathrm{~km} \mathrm{~s}^{-1}$ at the end of the simulation.

${ }^{\mathrm{e}}$ Mass of ${ }^{56} \mathrm{Ni}$ that has a velocity higher than $4000 \mathrm{~km} \mathrm{~s}^{-1}$ at the end of the simulation.

${ }^{\mathrm{f}}$ Mass of ${ }^{56} \mathrm{Ni}$ that has a velocity higher than $4700 \mathrm{~km} \mathrm{~s}^{-1}$ at the end of the simulation.

${ }^{g}$ Ratio of the values in the fifth to the third columns.

${ }^{\mathrm{h}}$ Here $v_{\mathrm{NS}}$ is the NS kick velocity estimated by Equation (7).

${ }^{i}$ Sequential serial number (model number) for Figure 11. The values of the fifth-eighth columns are plotted in Figure 11.

${ }^{\mathrm{j}}$ Number in parentheses denotes the power of 10 .

${ }^{\mathrm{k}}$ The values in the third and fourth columns (eighth column) could be overestimated (underestimated). See Section 4.2 for details.

factors of $\sim 1.5$ and 3 , respectively, compared with those calculated by a larger nuclear reaction network (464 nuclei are included). Then, for example, the mass of ejected ${ }^{56} \mathrm{Ni}$ and ${ }^{44} \mathrm{Ti}$ for the model b18.3-fid, $8.1 \times 10^{-2}$ and $7.4 \times 10^{-4} M_{\odot}$, could be translated as $5.4 \times 10^{-2}$ and $2.5 \times 10^{-4} M_{\odot}$, respectively, although the factors should depend on the inner structure of the progenitor models and the explosion asymmetries. Then, the obtained values of the masses of the ejected ${ }^{56} \mathrm{Ni}$ and ${ }^{44} \mathrm{Ti}$ are roughly consistent with the values suggested by the observations. The values of the masses of high-velocity ${ }^{56} \mathrm{Ni}$ listed in the fifth-seventh columns in Table 3 could also be overestimated, but the high-velocity ${ }^{56} \mathrm{Ni}$ is considered to be synthesized in outer, less neutron-rich regions. Then, the correction for the values in the fifth-seventh columns should be much smaller than that for the values in the third column. The values in the eighth column could be underestimated depending on the overestimation of the values in the third column. The correction factors themselves, however, are rather uncertain; hereafter, we proceed with discussion based on the values listed (directly calculated by the numerical code in this paper).

Hereafter, the effects of asymmetries of explosions on the matter mixing are explored. The parameters related to the asymmetry of an explosion are $\beta, \alpha$, and the type of asphericity of the explosion, i.e., "cos," "power," "exponential," or "elliptical" (see Equations (8)-(11), respectively, and Table 2). As seen in Figure 26 (in Appendix A), the larger the $\beta$ value, the higher the concentration of initial radial velocities along the polar ( $z$-axis), if the type of asphericity is fixed. It is noted that 

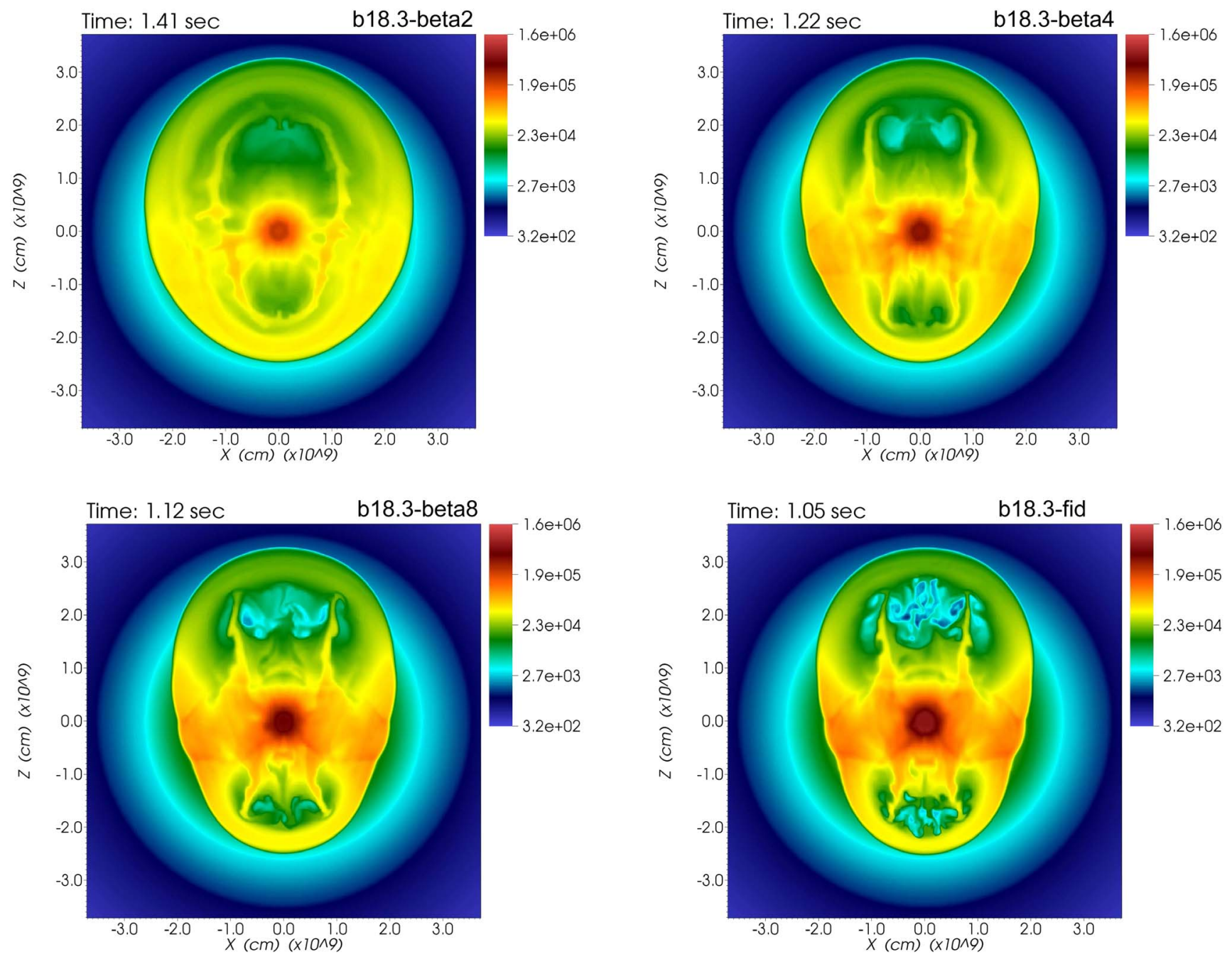

Figure 4. Density color maps (2D slices of the $x-z$ plane) in a logarithmic scale at an early phase of the explosion $(\sim 1 \mathrm{~s})$. The values in the color bars are in units of $\mathrm{g} \mathrm{cm}^{-3}$. The top left, top right, bottom left, and bottom right panels are for models b18.3-beta2, b18.3-beta4, b18.3-beta8, and b18.3-fid, respectively.

type "cos" was adopted in Papers I and II. As can be seen, the differences of the initial radial velocity distribution among $\beta=2,4,8$, and 16 are not so large around the polar axis, if the type is fixed to be "cos." In the order of "cos," "power," "exponential," and "elliptical," the concentration of radial velocities becomes higher. In this paper, type "elliptical" is adopted as the fiducial one (see Section 3.2). The dependence of the density distribution at an early phase $(\sim 1 \mathrm{~s})$ on each parameter related to the asphericity is discussed below. Figure 4 shows the dependence on the parameter $\beta$ (other parameters are fixed). The shape of the blast wave (the interface between red and green) slightly depends on the parameter $\beta$. As expected, the larger the $\beta$ value, the more elliptical the shape, but the elliptical shape is not so evident soon after the explosion compared with one of the initial radial velocity distributions seen in Figure 26

(in Appendix A). Inside the blast wave, the density distribution is more sensitive to $\beta$ than that for the outer part. High-density regions (red) for models with larger $\beta$ are more concentrated around the equatorial plane $(z=0)$. As can be seen, instabilities are developed in regions inside the blast wave (high-entropy bubbles; blue regions). The growth of instabilities at such an early phase may be due to Kelvin-Helmholtz (shear velocity is necessary for its growth) and/or RT instability. The larger the $\beta$ value, the stronger the growth of instabilities (see, in particular, the bottom two panels). In Figure 5, the dependence on the parameter $\alpha$ is presented. In the case of $\alpha=1.0$ (left panel), the shape of the blast wave is almost symmetric against the equatorial plane (as expected). On the other hand, in the case of $\alpha=2.0$ (right), the shape and extension are very different between the upper and lower regions. Compared with the case of $\alpha=1.0$, in the case of $\alpha=2.0$, there are the following features: the development of hydrodynamic instabilities and high-entropy bubbles is prominent in the upper regions, the blast wave reaches the radius of $3 \times 10^{9} \mathrm{~cm}$ earlier than in the case of $\alpha=1.0$ (see the time for each model), and there are high-density regions (red) in equatorial regions. Focusing on the regions disturbed by instabilities in the upper regions, the regions in the case of $\alpha=2.0$ are a bit larger than those in the case of $\alpha=1.0$, whereas lower-density regions (darker blue) are recognized in the case of $\alpha=1.0$. The features in the case of $\alpha=1.5$ (bottom left panel in Figure 4) are roughly in between the two cases above $(\alpha=1.0$ and 2.0). Figure 6 shows the dependence on the type of aspherical explosion. As can be seen, the shape of the blast wave is not so different among the four types, but the shape of type "elliptical" (bottom right) is slightly more elliptical. In the order b18.3-cos, b18.3-pwr, b18.3-exp, and b18.3-fid, the 

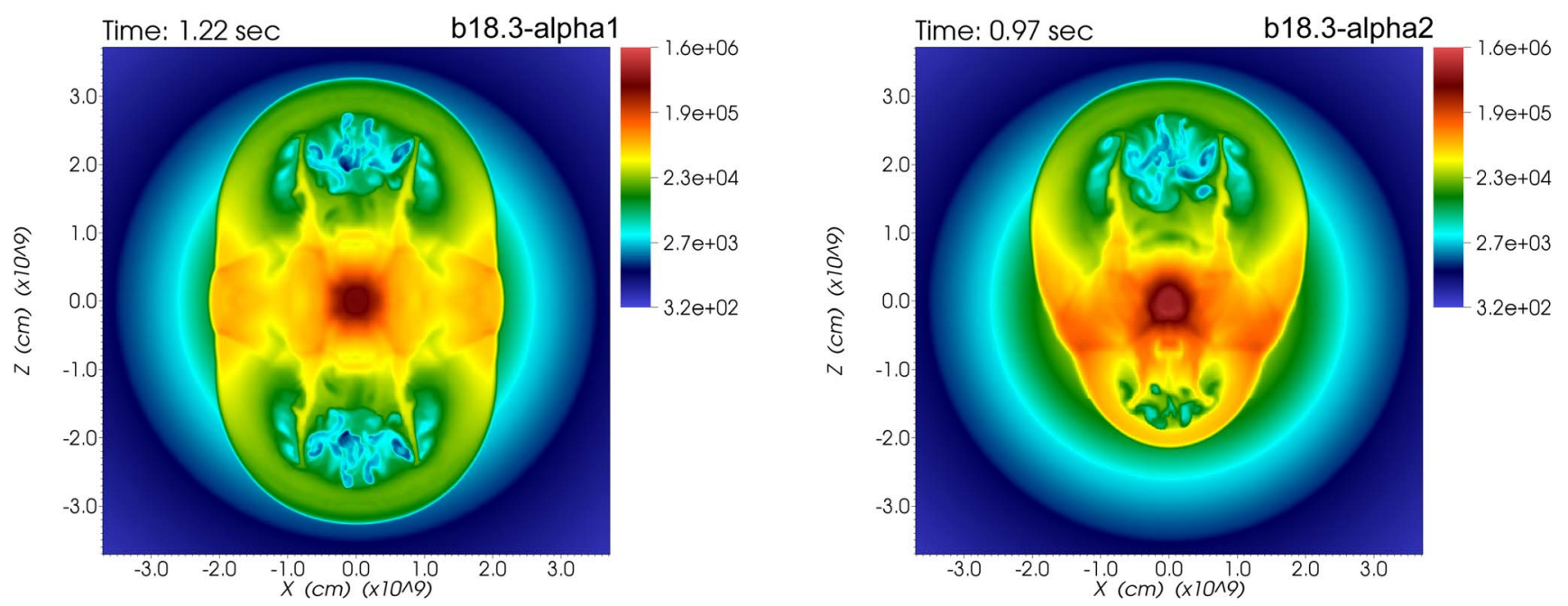

Figure 5. Same as Figure 4 but for models b18.3-alpha1 (left) and b18.3-alpha2 (right).
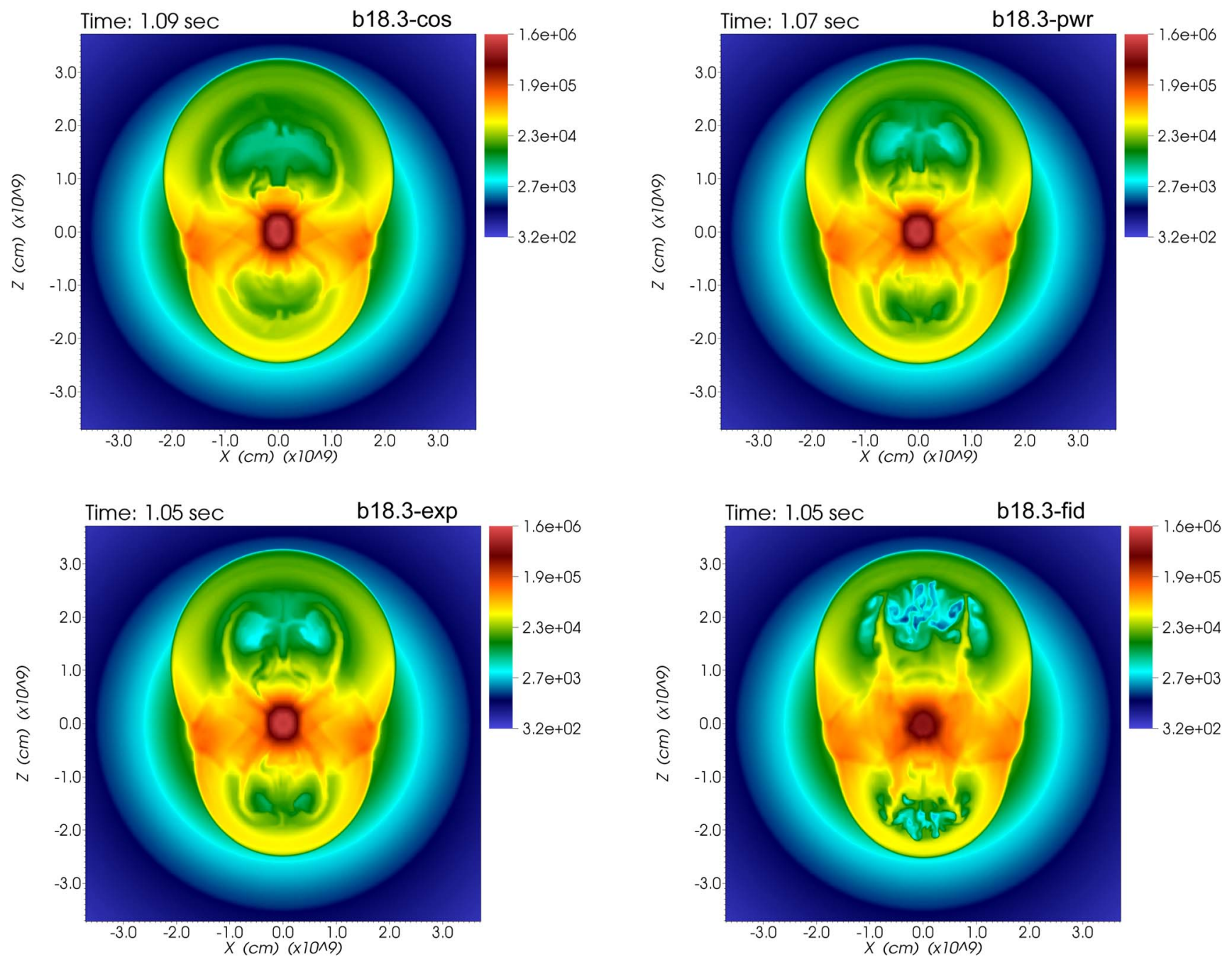

Figure 6. Same as Figure 4 but for models b18.3-cos (top left), b18.3-pwr (top right), b18.3-exp (bottom left), and b18.3-fid (bottom right).

regions of high-entropy bubbles inside the blast wave are more pronounced and disturbed due to instabilities. In Figure 7, the dependence of the early morphology of the explosion on the progenitor models is depicted. The shape of the blast wave is different between the two BSG models (b18.3 and n16.3; top panels) and the other RSG models (s18.0 and s19.8; bottom panels). At the time presented here, the blast wave is inside the $\mathrm{C}$ $+\mathrm{O}$ layer $\left(r<3 \times 10^{9} \mathrm{~cm}\right)$. As seen in Figure 1, the density structures are different among the progenitor models. The density structures are relatively similar between the two RSG 

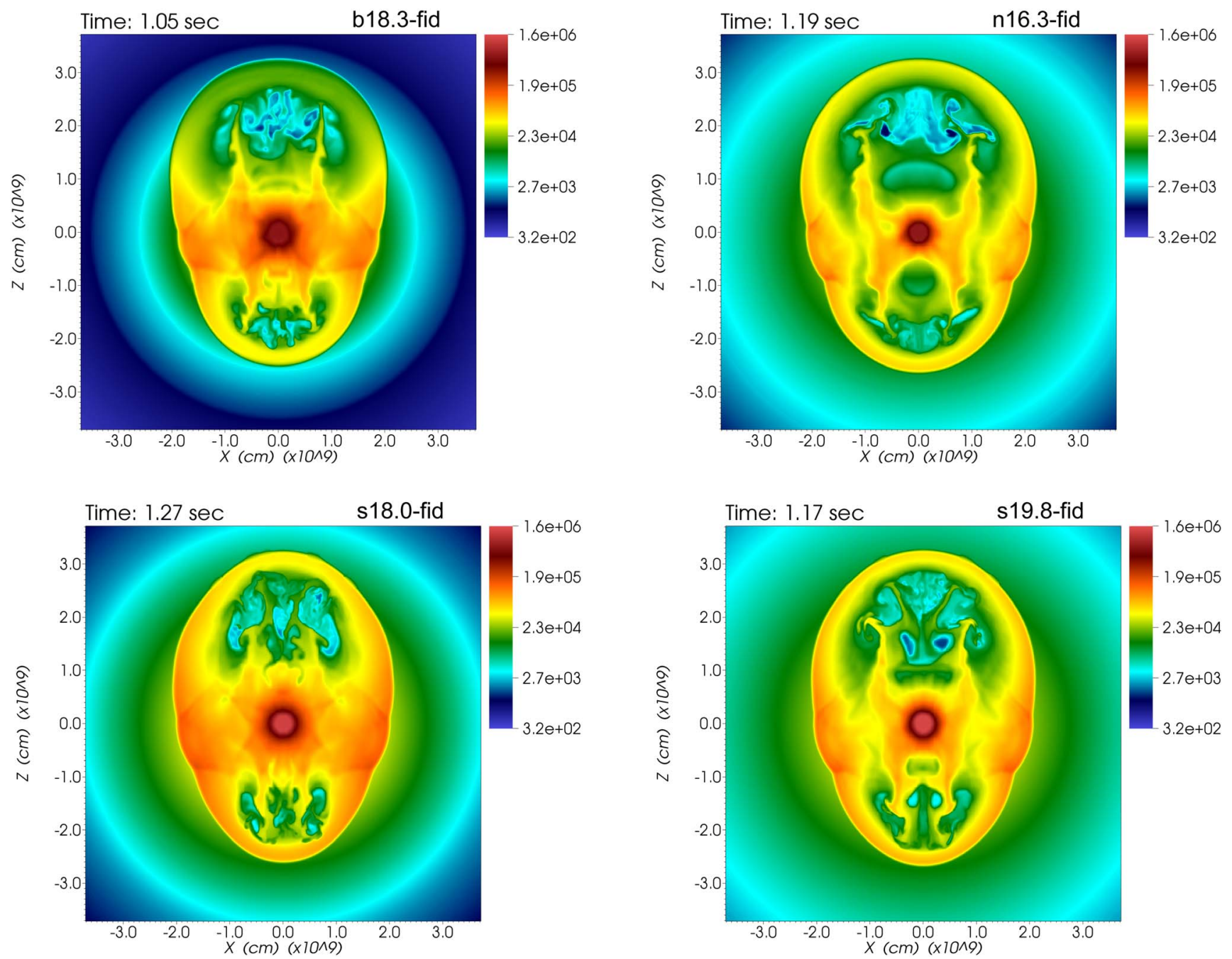

Figure 7. Same as Figure 4 but for models b18.3-fid (top left), n16.3-fid (top right), s18.0-fid (bottom left), and s19.8-fid (bottom right).

models, while the sizes (in both mass and radius; see Figures 1-3, respectively) of the $\mathrm{C}+\mathrm{O}$ cores and density gradients are different between the two BSG models. The size of the $\mathrm{C}+\mathrm{O}$ core of model b18.3 (binary merger model) is smaller than that of $n 16.3$ (single-star model). The $\rho r^{3}$ gradient in the $\mathrm{C}+\mathrm{O}$ core of model b18.3 is flatter than that of model n16.3. It is difficult to find a clear correlation between the morphology of the explosion at the early phase and the density structure inside the $\mathrm{C}+\mathrm{O}$ core. Nevertheless, the bipolar structure for the two RSG models is more prominent (the width of the bipolar structure is narrower) than that for the two BSG models. Between the BSG models, the shape of the bipolar structure of model n16.3 is wider than that of b18.3 because of the steeper $\rho r^{3}$ gradient and the larger size of the $\mathrm{C}+\mathrm{O}$ core, which causes rapid deceleration of the shock wave. Among the four pre-supernova models, model n16.3 has a distinct $\rho r^{3}$ profile inside the silicon layer compared with those of the others; i.e., the profile of the silicon layer of n16.3 is rather flat compared with those of the others (the gradients of $\rho r^{3}$ for the others are overall negative), which causes the deceleration of the earliest phase. Then, the structures of the silicon layer could affect the morphologies of the early phases.

Hereafter, spatial distributions of representative elements are presented. Figure 8 shows the distributions of ${ }^{56} \mathrm{Ni}$ at an early phase $(\sim 1 \mathrm{~s})$. The dependence on the progenitor model is presented. The shapes of the outer edge of the distribution of
${ }^{56} \mathrm{Ni}$ are not so different among the progenitor models, although the widths of the bipolar structure are slightly different, reflecting the density distribution as seen in Figure 7. The inner distributions of ${ }^{56} \mathrm{Ni}$ are rather different among the models. Hole structures (cavities) of ${ }^{56} \mathrm{Ni}$ inside the outer edge are found in models b18.3 and s19.8. It is noted that the small spherical holes $\left(r \lesssim 10^{8} \mathrm{~cm}\right)$ at the origin are the regions corresponding to the compact object. The products of the explosive nucleosynthesis sensitively depend on the peak temperature and density during the burning process (see, e.g., Jerkstrand et al. 2015). In a high-entropy regime, the synthesis of ${ }^{56} \mathrm{Ni}$ is limited due to the so-called alpha-rich freeze-out. The cavities inside the outer edges could correspond to the regions of strong alpha-rich freeze-out. Figure 9 shows the distributions of ${ }^{56} \mathrm{Ni}$ just before the shock breakout. Depending on the structures of the progenitor models, the distributions are rather different. In the two RSG models s18.0 and s19.8, a bicone-like structure is clearly seen. On top of the bicone-like structures, small-scale fingers due to RT instabilities are prominent. Between the two BSG models, b18.3 and n16.3, the distribution of ${ }^{56} \mathrm{Ni}$ in model b18.3 is more shrunken and wobbling than that in model n16.3. Comparing the distributions of ${ }^{56} \mathrm{Ni}$ in Figures 8 and 9, the initial bipolar-like distributions are roughly kept even just before the shock breakout, but the shapes are rather modified during the shock propagation. 

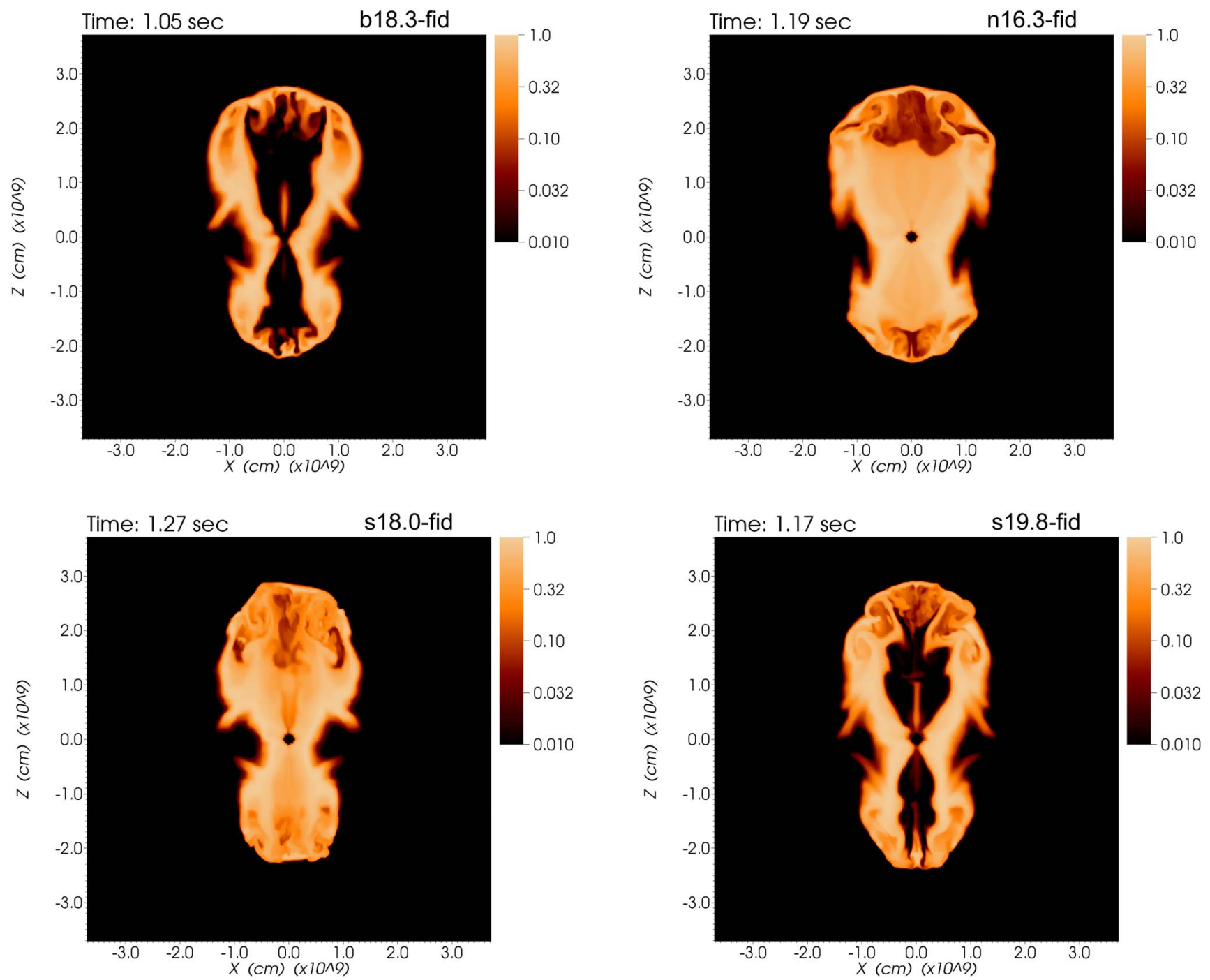

Figure 8. Color maps (2D slices of the $x-z$ plane) of the mass fraction of ${ }^{56} \mathrm{Ni}$ at an early phase of the explosion $(\sim 1 \mathrm{~s})$. The colors are logarithmically scaled. The top left, top right, bottom left, and bottom right panels are for models b18.3-fid, n16.3-fid, s18.0-fid, and s19.8-fid, respectively.

Figure 10 shows the $3 \mathrm{D}$ distribution of elements ${ }^{56} \mathrm{Ni},{ }^{28} \mathrm{Si},{ }^{16} \mathrm{O}$, and ${ }^{4} \mathrm{He}$ just before the shock breakout. The dependence on the progenitor model is shown. The distributions of ${ }^{56} \mathrm{Ni}$ are different from each other (as also seen in Figure 9), and other elements, ${ }^{28} \mathrm{Si},{ }^{16} \mathrm{O}$, and ${ }^{4} \mathrm{He}$, are also different from each other. The distributions in the two RSG models (s18.0-fid and s19.8fid) are similar to each other, but the distributions in the two BSG models (b18.3-fid and n16.3-fid) are rather different. Overall, the distributions of two heavier elements, i.e., ${ }^{56} \mathrm{Ni}$ and ${ }^{28} \mathrm{Si}$, are similar to each other compared with the other two elements, ${ }^{16} \mathrm{O}$ and ${ }^{4} \mathrm{He}$. In models $\mathrm{n} 16.3$, s18.0, and s19.8, bicone-like structures of ${ }^{56} \mathrm{Ni}$ and ${ }^{28} \mathrm{Si}$ are seen. The bicone-like structures in model n16.3 are more asymmetric against the equatorial plane ( $x-y$ plane). A distinct feature of model b18.3fid is that the distributions of ${ }^{16} \mathrm{O}$ and ${ }^{4} \mathrm{He}$ are more concentrated around the equatorial plane (the fingers are extended from more central regions) than those in the other models. On the other hand, the distributions of ${ }^{16} \mathrm{O}$ and ${ }^{4} \mathrm{He}$ in models $\mathrm{n} 16.3$, s18.0, and s19.8 are more roundly extended around the elements ${ }^{56} \mathrm{Ni}$ and ${ }^{28} \mathrm{Si}$. The reason for the different distributions of ${ }^{16} \mathrm{O}$ and ${ }^{4} \mathrm{He}$ among the progenitor models is discussed in Section 4.3.
As introduced in Section 1, the observations of SN 1987A suggest the existence of high-velocity ${ }^{56} \mathrm{Ni}$ in the helium and hydrogen layers, which has led to the invocation of matter mixing to convey the innermost material into the outer layers. From the observations of [Fe II] lines (Haas et al. 1990), the tails of the lines reach $\sim 4000 \mathrm{~km} \mathrm{~s}^{-1}$, and at least $4 \%$ of the iron had a velocity of $\gtrsim 3000 \mathrm{~km} \mathrm{~s}^{-1}$; from the fine structure developed in the $\mathrm{H}_{\alpha}$ line (the Bochum event; Hanuschik et al. $1988)$, the existence of a high-velocity $\left(4700 \pm 500 \mathrm{~km} \mathrm{~s}^{-1}\right)$ ${ }^{56} \mathrm{Ni}$ clump of $\sim 10^{-3} M_{\odot}$ has been suggested (Utrobin et al. 1995). Such observational constraints can be a test for the models in this paper. Then, we consider three conditions to test the models, as follows: (i) the ratio of the mass of ${ }^{56} \mathrm{Ni}$ that has a velocity $\geqslant 3000 \mathrm{~km} \mathrm{~s}^{-1}$ to the total ${ }^{56} \mathrm{Ni}$ mass is greater than $4 \%$; (ii) the mass of ${ }^{56} \mathrm{Ni}$ that has a velocity $\geqslant 4000 \mathrm{~km} \mathrm{~s}^{-1}$ is greater than $10^{-3} M_{\odot}$; and (iii) the mass of ${ }^{56} \mathrm{Ni}$ that has a velocity $\geqslant 4700 \mathrm{~km} \mathrm{~s}^{-1}$ is greater than $10^{-3} M_{\odot}$. The first condition is based on Haas et al. (1990). For the second condition, there has been no clear constraint on the mass, but we take $\sim 10^{-3} M_{\odot}$ as a minimum requirement based on the fact that the tails of the [Fe II] line reach $4000 \mathrm{~km} \mathrm{~s}^{-1}$ and the suggestion from Utrobin et al. (1995). The third condition is directly based on Utrobin et al. (1995) and more stringent than 

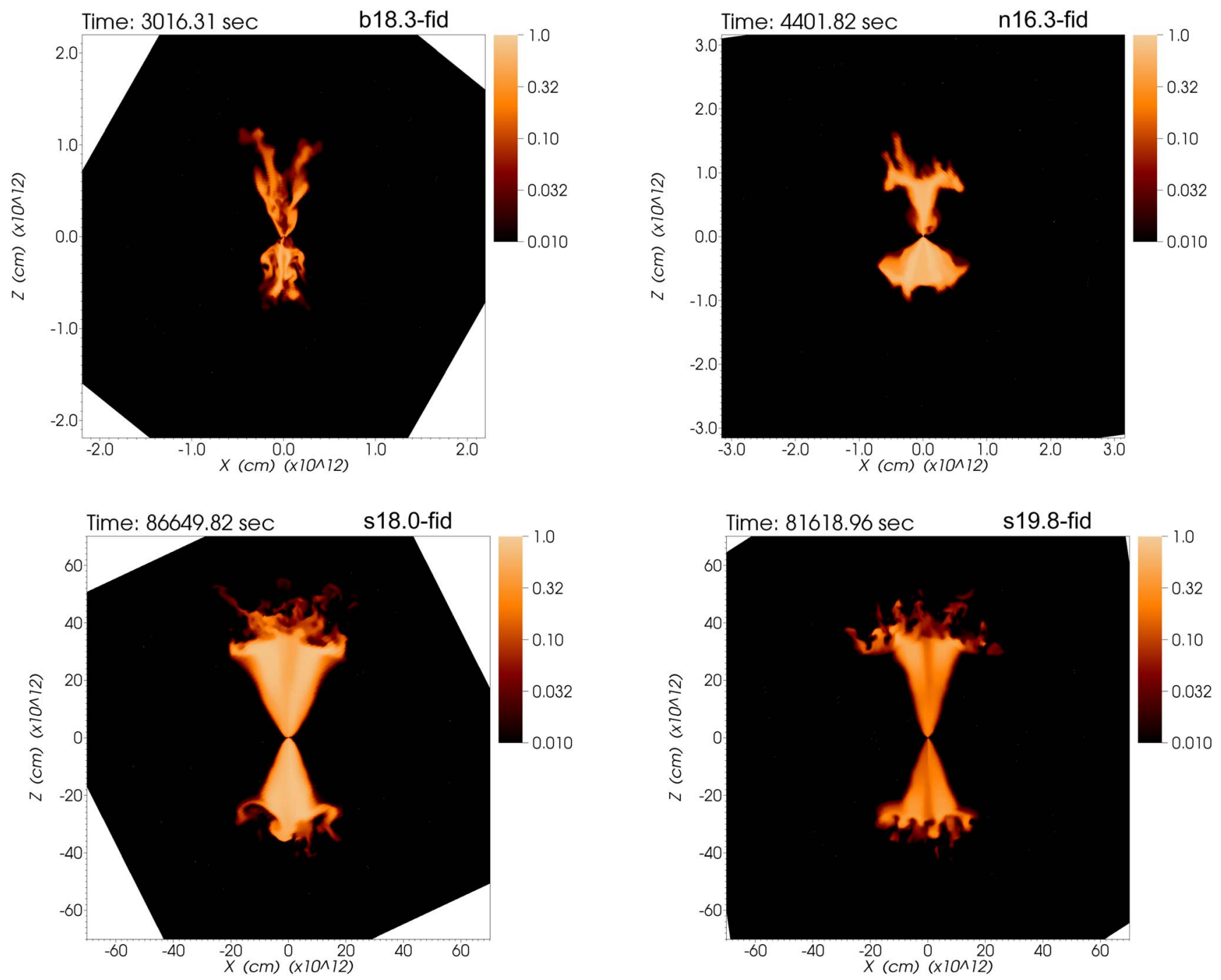

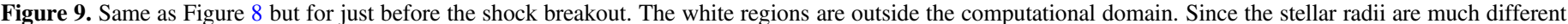

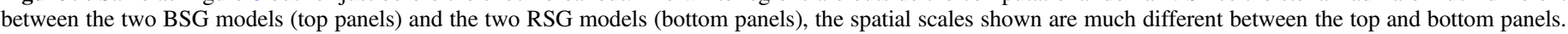

the second one. The derivation of the values in Utrobin et al. (1995) was, however, based on a simple modeling of the $\mathrm{H}_{\alpha}$ line, and the errors in the velocity are bit large. Then, we regard the third condition as an optional one. From the calculated models, the masses of the representative elements, in particular ${ }^{56} \mathrm{Ni}$, and their radial velocities are discussed by comparing with the conditions above. In Table 3 , the masses of ${ }^{56} \mathrm{Ni}$ that have radial velocities greater than specific values are listed in the fifth, sixth, and seventh columns, i.e., $M_{3.0}\left({ }^{56} \mathrm{Ni}\right), M_{4.0}$ $\left({ }^{56} \mathrm{Ni}\right)$, and $M_{4.7}\left({ }^{56} \mathrm{Ni}\right)$, respectively. The second and third conditions can be tested by seeing the sixth and seventh columns. The first condition can be tested from the eighth column, $M_{3.0} / M_{\mathrm{ej}}\left({ }^{56} \mathrm{Ni}\right)$. In Figure 11 , the values of the fiftheighth columns for all models listed in Table 3 are plotted (see Figure 11 for the discussion in this section and Section 4.3, when necessary). The models of lower-resolution simulations that satisfy both the first and second conditions are b18.3-beta8, b18.3-fid, s18.0-fid, s19.8-beta4, s19.8-beta8, s19.8-fid, b18.3alpha2, b18.3-clp0, and b18.3-ein3.0. It is worth noting that there is no model with the n16.3 progenitor that satisfies the two conditions simultaneously. The models that include "mo13" have the same values for the parameters $\beta$ and $\alpha$ as in Paper I, but among the models, there is no model that satisfies the two conditions. Only three models, i.e., s19.8beta8, b18.3-alpha2, and b18.3-ein3.0, satisfy not only the two conditions but also the third one. The dependence of the radial velocity of ${ }^{56} \mathrm{Ni}$ on the parameter $\beta \equiv v_{\mathrm{pol}} / \nu_{\mathrm{eq}}$ can be checked by comparing models, e.g., b18.3-beta2, b18.3-beta4, b18.3beta8, and b18.3-fid. The larger the $\beta$ value, the larger the values $M_{4.0}\left({ }^{56} \mathrm{Ni}\right)$ and $M_{3.0} / M_{\mathrm{ej}}\left({ }^{56} \mathrm{Ni}\right)$. It is noted that the larger the $\beta$ value, the stronger the concentration of initial radial velocities around the polar axis (see Figure 26 in Appendix A). The dependence on the type of asphericity can be seen by comparing models b18.3-fid, b18.3-cos, b18.3-pwr, and b18.3exp. In the order of b18.3-cos, b18.3-pwr, b18.3-exp, and b18.3-fid, the values $M_{4.0}\left({ }^{56} \mathrm{Ni}\right)$ and $M_{3.0} / M_{\mathrm{ej}}\left({ }^{56} \mathrm{Ni}\right)$ increase. As seen in Figure 26, in type "elliptical," the concentration of initial radial velocities around the polar axis is most prominent if the $\beta$ values are fixed. Compared with b18.3-fid, model b18.3-alpha2 has larger $M_{4.0}\left({ }^{56} \mathrm{Ni}\right)$ and $M_{3.0} / M_{\mathrm{ej}}\left({ }^{56} \mathrm{Ni}\right)$ values, which reflects a stronger explosion in a certain direction in model b18.3-alpha2 with $\alpha \equiv v_{\text {up }} / v_{\text {down }}=2.0$. The $M_{4.0}$ $\left({ }^{56} \mathrm{Ni}\right)$ and $M_{3.0} / M_{\text {ej }}\left({ }^{56} \mathrm{Ni}\right)$ values in models b18.3-fid and b18.3-clp0 are not so different from each other; however, the values in model b18.3-clp0 (no fluctuation in the initial radial velocities; see Appendix B) are slightly larger than those in 


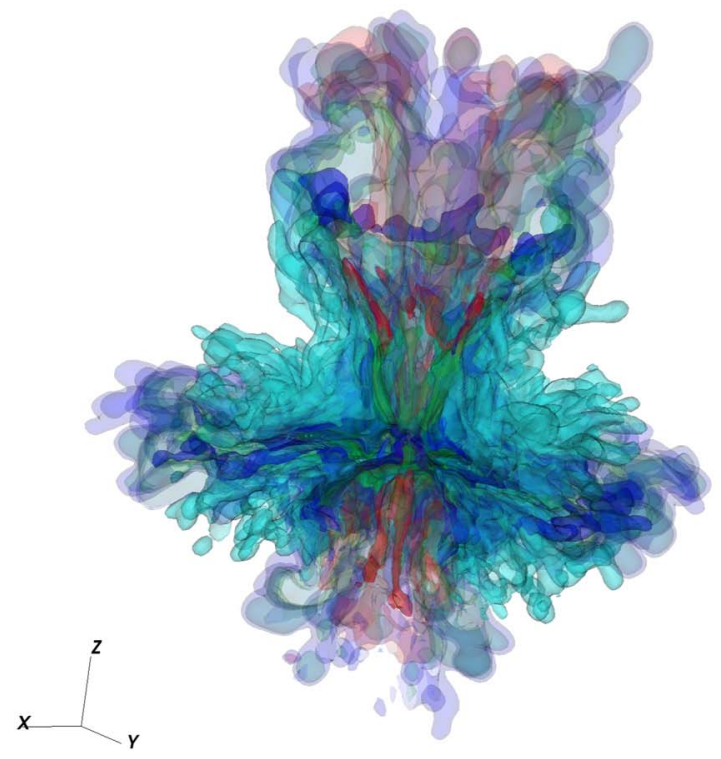

Time: $86649.82 \mathrm{sec}$

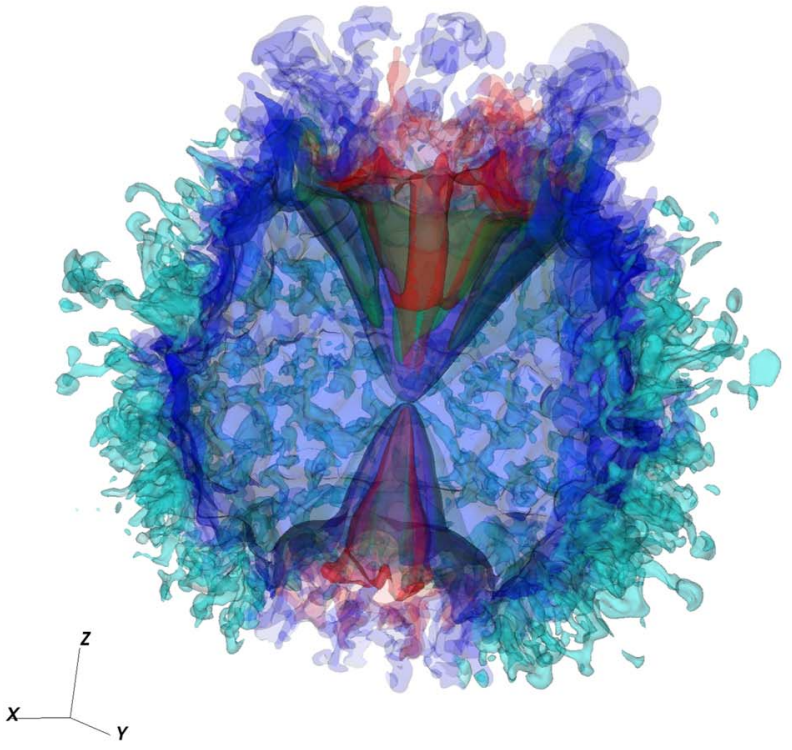

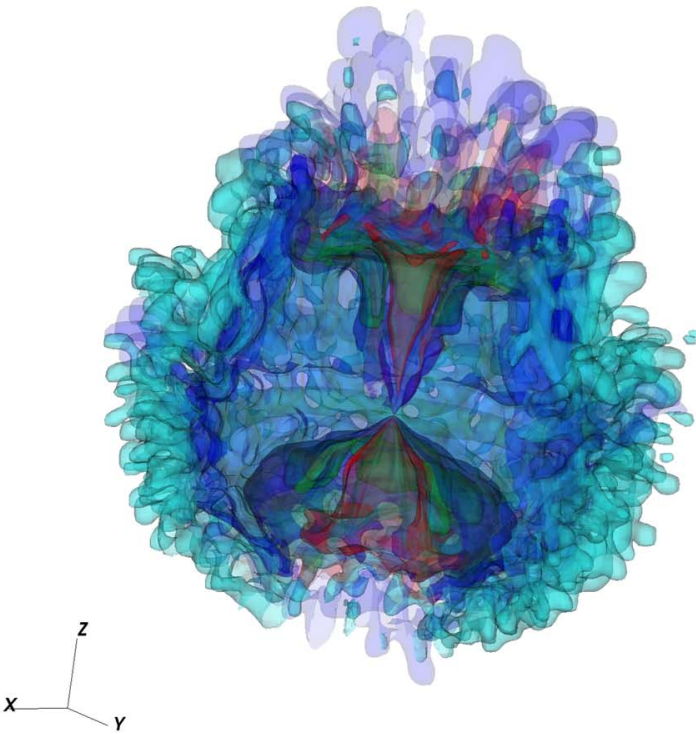

Time: $81618.96 \mathrm{sec}$

s19.8-fid

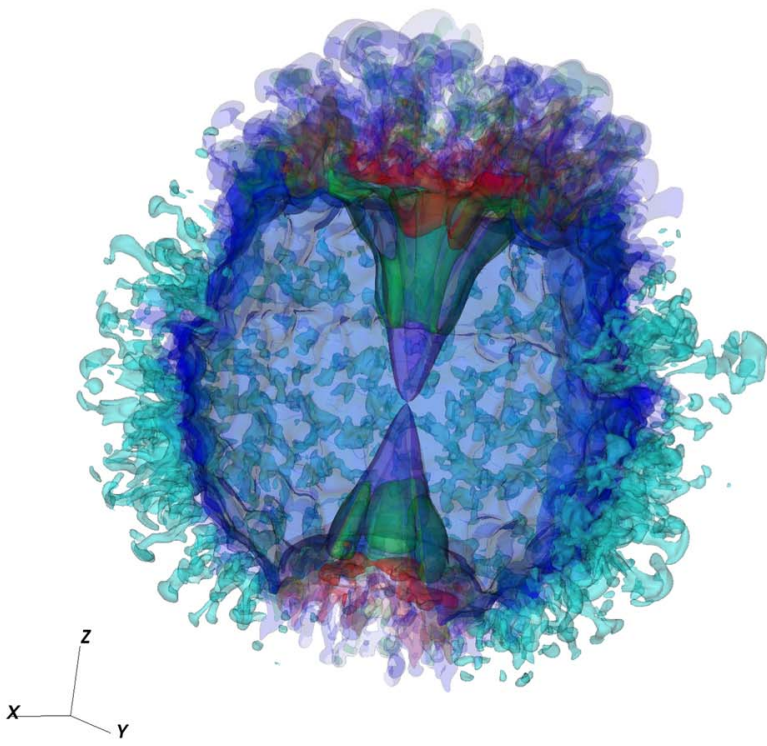

Figure 10. Isosurfaces of the mass fractions of elements, ${ }^{56} \mathrm{Ni}$ (red), ${ }^{28} \mathrm{Si}$ (green), ${ }^{16} \mathrm{O}$ (blue), and ${ }^{4} \mathrm{He}$ (light blue), just before the shock breakout in a $3 \mathrm{D}$ view. Isosurfaces of $10 \%$ (lighter color) and 70\% (darker color) of the maximum value for each element are shown. To see the inner structure, the regions of $x>0$ and $y>0$ are clipped. The top left, top right, bottom left, and bottom right panels are for models b18.3-fid, n16.3-fid, s18.0-fid, and s19.8-fid, respectively. Interactive 3D models on Sketchfab corresponding to the top left (https://skfb.ly/6OZDr), top right (https://skfb.ly/6OZD8), bottom left (https://skfb.ly/6OZDt), and bottom right (https://skfb.ly/6OZD9) panels are available.

model b18.3-fid. Then, the existence of the initial clumpiness (the fluctuations in the initial radial velocities) has a negative role, at least for the b18.3 model. The role of initial clumpiness could, however, change depending on the structure of the progenitor model. Actually, in Paper I, the existence of an initial clumpiness has a positive role in obtaining high-velocity ${ }^{56} \mathrm{Ni}$ with the $\mathrm{n} 16.3$ model (see the results for models AM2 and AM3 in Paper I). As a summary, if we exclude models with RSG progenitor models and/or the highest $E_{\text {in }}$ value $\left(3.0 \times 10^{51} \mathrm{erg}\right)$, models b18.3-beta8, b18.3-fid, b18.3-alpha2, and b18.3-clp0 could be promising for SN 1987A at this time.
Here the mass distributions of representative elements, including ${ }^{56} \mathrm{Ni}$, are discussed. Figure 12 shows the distribution of elements as a function of radial velocity at the end of the simulation (see the figure caption for the definitions of several variables). The dependence on the parameter $\beta$ is shown. As can be seen, helium and hydrogen in the outer layers have very high velocities of $\gtrsim 6000 \mathrm{~km} \mathrm{~s}^{-1}$, and the distribution of elements ${ }^{1} \mathrm{H}$, ${ }^{4} \mathrm{He},{ }^{12} \mathrm{C},{ }^{16} \mathrm{O}$, and ${ }^{28} \mathrm{Si}$ in velocities $\gtrsim 5000 \mathrm{~km} \mathrm{~s}^{-1}$ hardly depends on the $\beta$ value. On the other hand, the distributions in velocities of $\lesssim 5000 \mathrm{~km} \mathrm{~s}^{-1}$ are different among the four models. The most distinct feature is that the larger the $\beta$ value, the more 

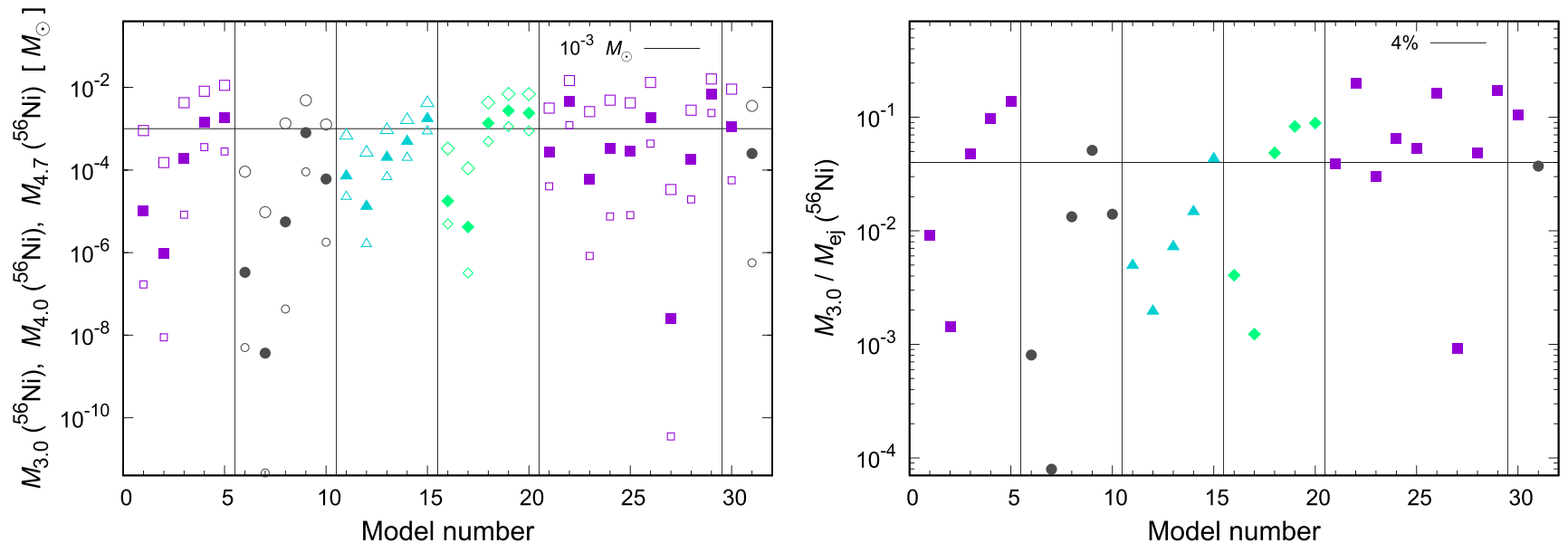

Figure 11. Left panel: masses of ${ }^{56} \mathrm{Ni}$ that have velocities higher than 3000,4000 , and $4700 \mathrm{~km} \mathrm{~s}^{-1}$ at the end of the simulation, i.e., $M_{3.0}\left({ }^{56} \mathrm{Ni}\right)($ large open points), $M_{4.0}\left({ }^{56} \mathrm{Ni}\right.$ ) (filled points), and $M_{4.7}\left({ }^{56} \mathrm{Ni}\right)$ (small open points), respectively, as a function of the model number (see the 10th column in Table 3). Squares, circles, triangles, and diamonds denote the points for the models with the progenitor models b18.3, n16.3, s18.0, and s19.8, respectively. The horizontal solid line is the value of $10^{-3} M_{\odot}$. Right panel: ratio of the mass of ${ }^{56} \mathrm{Ni}$ that has a velocity higher than $3000 \mathrm{~km} \mathrm{~s}^{-1}$ to the total ejected ${ }^{56} \mathrm{Ni}$ mass at the end of the simulation, $M_{3.0} / M_{\mathrm{ej}}$ $\left({ }^{56} \mathrm{Ni}\right)$, as a function of the model number. The four shapes are the same as in the left panel. The horizontal solid line is the value of $4 \times 10^{-2}(4 \%)$.
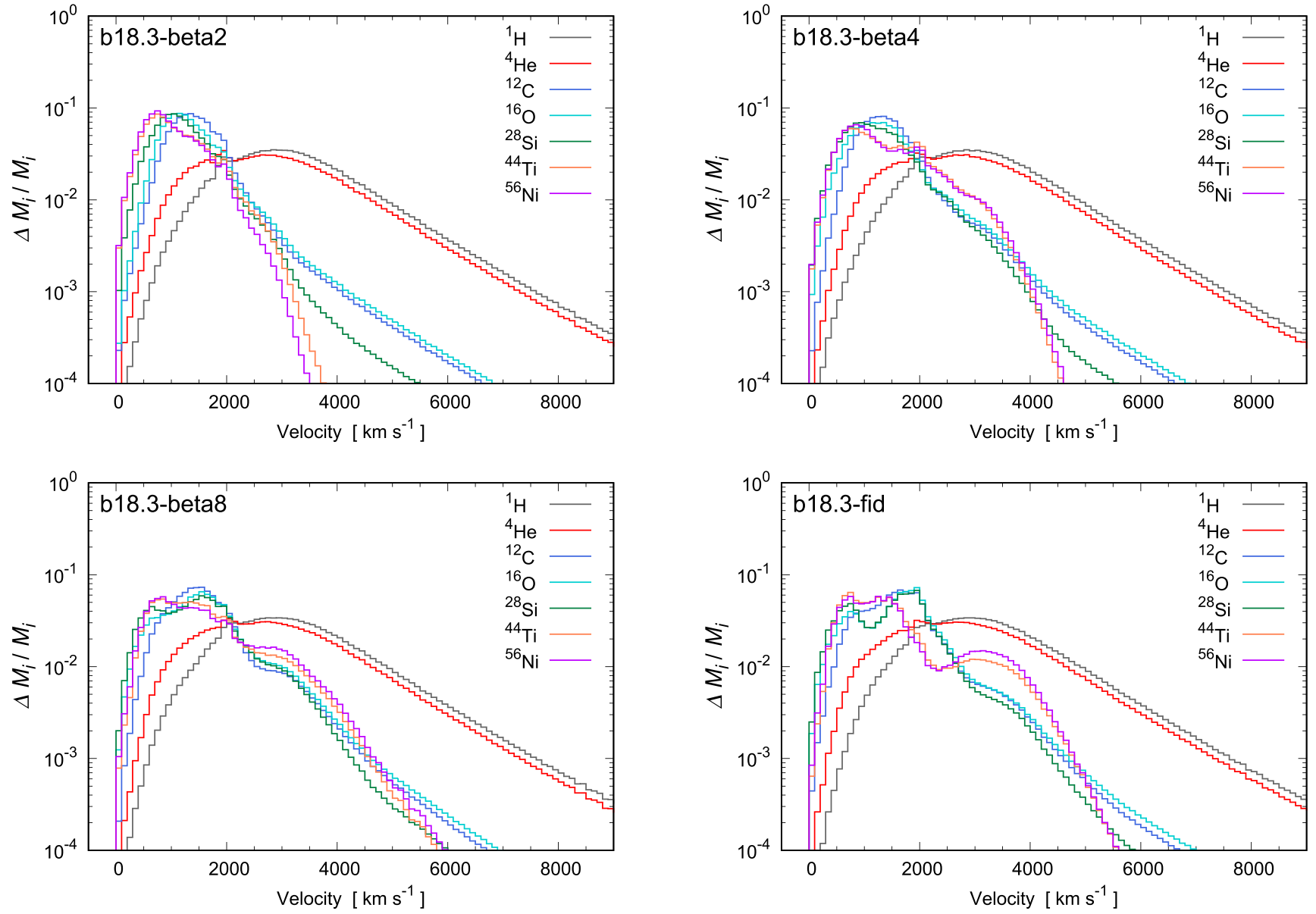

Figure 12. Normalized masses of elements ${ }^{1} \mathrm{H},{ }^{4} \mathrm{He},{ }^{12} \mathrm{C},{ }^{16} \mathrm{O},{ }^{28} \mathrm{Si},{ }^{44} \mathrm{Ti}$, and ${ }^{56} \mathrm{Ni}$ as a function of radial velocity at the end of the simulation (hereafter, the times in parentheses after the model names denote the simulation time corresponding to each model shown). The top left, top right, bottom left, and bottom right panels are for models b18.3-beta2 (76,819 s), b18.3-beta4 (78,501 s), b18.3-beta8 (78,128 s), and b18.3-fid (80,621 s), respectively. Here $\Delta M_{i}$ is the mass of the element, $i$, in the velocity range of $v \sim v+\Delta v$, and $M_{i}$ is the total ejected mass of the element, $i$. The size of the velocity bins, $\Delta v$, is $100 \mathrm{~km} \mathrm{~s}^{-1}$.

extended $\left(\gtrsim 4000 \mathrm{~km} \mathrm{~s}^{-1}\right)$ the high-velocity tails of ${ }^{56} \mathrm{Ni}$ and ${ }^{44} \mathrm{Ti}$. The amounts of ${ }^{12} \mathrm{C},{ }^{16} \mathrm{O}$, and ${ }^{28} \mathrm{Si}$ around $4000 \mathrm{~km} \mathrm{~s}^{-1}$ are also more enhanced than those for models with higher $\beta$ values. It is notable that inward mixing of hydrogen down to the velocity of $\sim 1000 \mathrm{~km} \mathrm{~s}^{-1}$ is recognized, as seen in Figure 12. The minimum velocities of hydrogen are comparable with the values (e.g., $800 \mathrm{~km} \mathrm{~s}^{-1}$; Shigeyama \& Nomoto 1990) suggested by modeling of the light curves of SN 1987A (Shigeyama \& Nomoto 1990; 

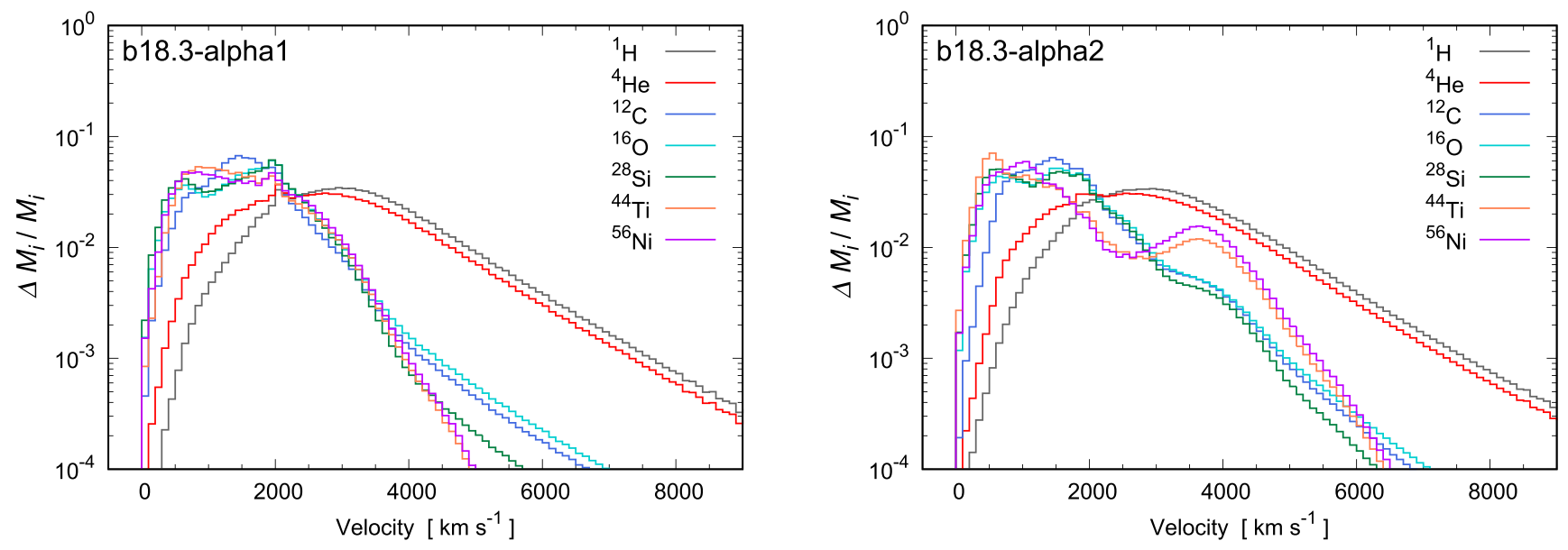

Figure 13. Same as Figure 12 but for models b18.3-alpha1 (left; 77,425 s) and b18.3-alpha2 (right; 77,261 s).
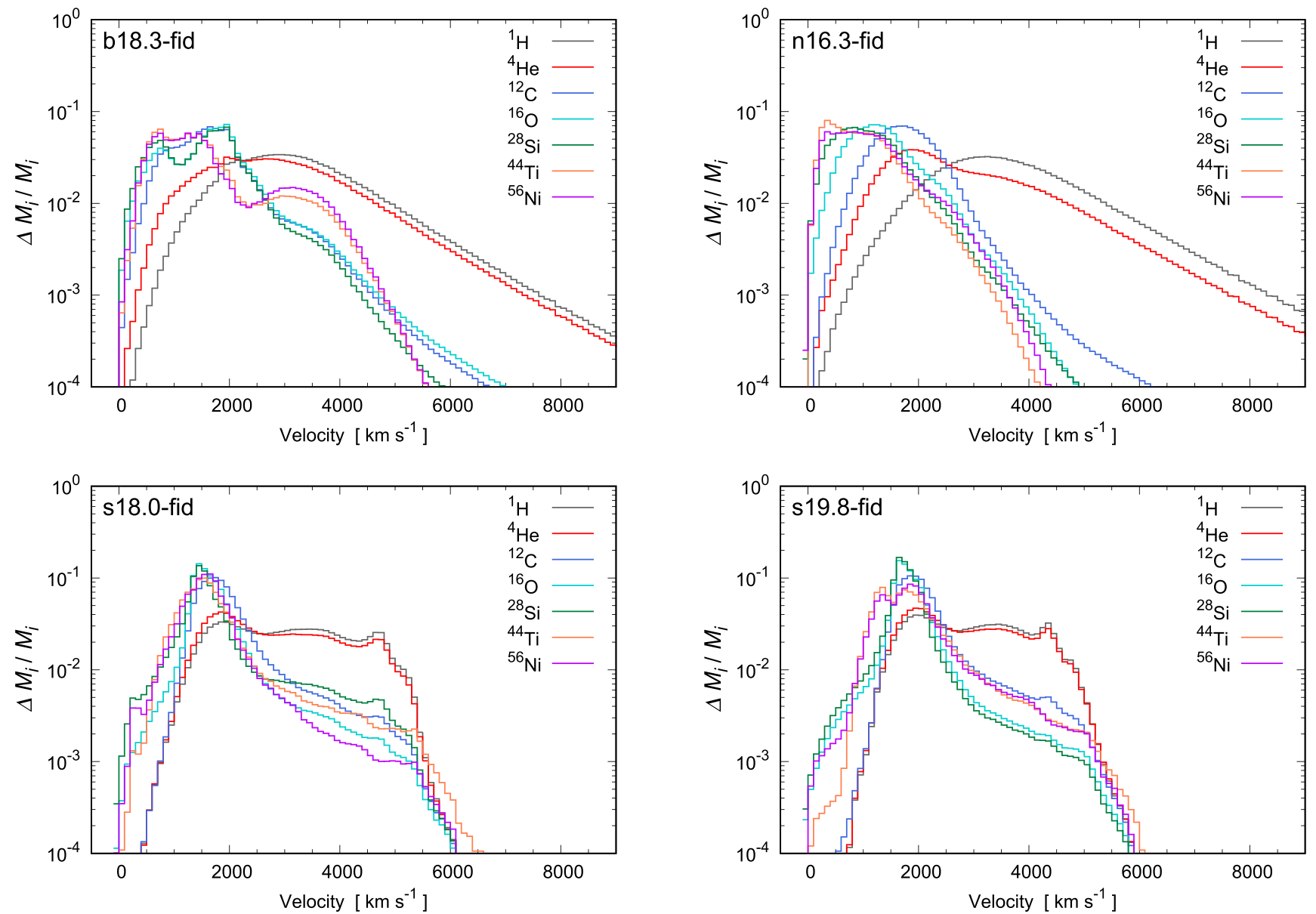

Figure 14. Same as Figure 12 but for models b18.3-fid (top left; 80,621 s), n16.3-fid (top right; 85,714 s), s18.0-fid (bottom left; 279,037 s), and s19.8-fid (bottom right; $283,161 \mathrm{~s})$.

Blinnikov et al. 2000), and the values are marginally consistent with the value $\left(\lesssim 700 \mathrm{~km} \mathrm{~s}^{-1}\right)$ deduced from the spectral modeling (Kozma \& Fransson 1998). As mentioned above, among the models displayed in Figure 12, only models b18.3beta8 (bottom left) and b18.3-fid (bottom right) have the amount of high-velocity ${ }^{56} \mathrm{Ni}$ required from the observations. In Figure 13, the dependence on the parameter $\alpha$ is presented. As for ${ }^{1} \mathrm{H}$ and ${ }^{4} \mathrm{He}$, the distributions are similar between the two cases ( $\alpha=1.0$ and 2.0). On the other hand, the distributions for other elements, ${ }^{12} \mathrm{C},{ }^{16} \mathrm{O},{ }^{28} \mathrm{Si},{ }^{44} \mathrm{Ti}$, and ${ }^{56} \mathrm{Ni}$, are rather different between the two cases, in particular for velocities of $\gtrsim 2000 \mathrm{~km} \mathrm{~s}^{-1}$. Compared with the case of $\alpha=1.0$, in the case of $\alpha=2.0$, the amount of elements ${ }^{12} \mathrm{C},{ }^{16} \mathrm{O}$, and ${ }^{28} \mathrm{Si}$ around velocities of about $2000 \mathrm{~km} \mathrm{~s}^{-1}$ is slightly reduced, whereas the amount around $4000 \mathrm{~km} \mathrm{~s}^{-1}$ is enhanced. Such features are more prominent for elements ${ }^{44} \mathrm{Ti}$ and ${ }^{56} \mathrm{Ni}$. In the case of $\alpha=2.0$, a bump around velocities of $2500-3000 \mathrm{~km} \mathrm{~s}^{-1}$ is recognized for ${ }^{44} \mathrm{Ti}$ and ${ }^{56} \mathrm{Ni}$, and the second peak appears at about $3500 \mathrm{~km} \mathrm{~s}^{-1}$. 


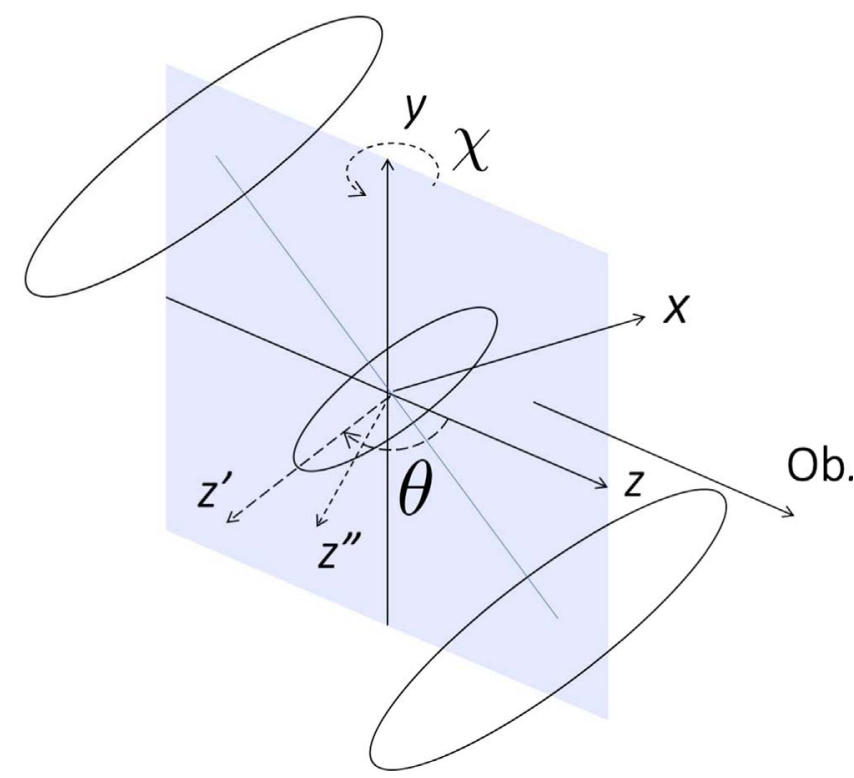

Figure 15. Schematic picture for an assumed direction of the bipolar-like explosion axis to observers on Earth and the triple ring structure (for the configuration of the triple ring structure to observers, see, e.g., Sugerman et al. 2005a, 2005b; Tziamtzis et al. 2011). In order to see the impact of the direction of the explosion axis on the line-of-sight velocity of ${ }^{56} \mathrm{Ni}$, the simulation box (initially, the explosion axis is directed to the $z$-axis) is rotated for the estimation. First, the $z$-axis in the simulation box is set to be directed to the observers on Earth. Then, the simulation box is rotated around the original $x$-axis by an angle of $\theta$ (the $z$-axis is rotated to be the $z^{\prime}$-axis). Finally, the box is rotated around the original $y$-axis by an angle of $\chi$ (the $z^{\prime}$-axis is rotated to be the $z^{\prime}$-axis).

In the case of $\alpha=2.0$, the high-velocity tail for elements ${ }^{44} \mathrm{Ti}$ and ${ }^{56} \mathrm{Ni}$ is extended to velocities greater than $4000 \mathrm{~km} \mathrm{~s}^{-1}$. In the middle case ( $\alpha=1.5$ ) between the two cases (see the bottom right panel in Figure 12), the peak for elements ${ }^{12} \mathrm{C},{ }^{16} \mathrm{O}$, and ${ }^{28} \mathrm{Si}$ around $2000 \mathrm{~km} \mathrm{~s}^{-1}$ is more prominent than for the case of $\alpha=2.0$, whereas the second peak, for elements ${ }^{44} \mathrm{Ti}$ and ${ }^{56} \mathrm{Ni}$, is shifted to a bit lower-velocity regions and is broader than for the case of $\alpha=2.0$. Figure 14 shows the dependence on the progenitor model. As seen in the figure, there are significant differences between the two BSG models (top panels) and the two RSG models (bottom panels). In the two RSG models, the maximum velocities of the elements are apparently limited to around $\sim 5000 \mathrm{~km} \mathrm{~s}^{-1}$, which is in contrast to the BSG models. This feature in the RSG models is attributed to the structures of the extended $\left(\gtrsim 6 \times 10^{13} \mathrm{~cm}\right)$ hydrogen envelopes. The blast wave is continuously decelerated during the propagation in the extended hydrogen envelope in the RSG models. Another feature is that even the innermost elements, ${ }^{56} \mathrm{Ni}$ and ${ }^{44} \mathrm{Ti}$, finally reach the highest-velocity regions $\left(\sim 5000 \mathrm{~km} \mathrm{~s}^{-1}\right)$, although the amounts are not so significant, in particular, in model s18.0-fid. Among the two BSG models, there is a clear difference in the extension of the high-velocity tails of ${ }^{56} \mathrm{Ni}$ and ${ }^{44} \mathrm{Ti}$. In model b18.3-fid (top left), a bump is present around $\lesssim 2000-2500 \mathrm{~km} \mathrm{~s}^{-1}$, and the tail is more extended than that in model n16.3-fid.

So far, only radial velocities of elements are discussed, but the observed [Fe II] lines (Haas et al. 1990) involved with the line-of-sight velocity of iron (the decay product of ${ }^{56} \mathrm{Ni}$ ) should also be explained from the models. Based on the simulation results, by changing the direction of the axis of the bipolar-like explosion (z-axis in the simulation box) to observers on Earth, distributions of the ${ }^{56} \mathrm{Ni}$ mass in the line-of-sight velocity are estimated. Figure 15 shows a schematic picture for an assumed direction of the axis of the bipolar-like explosion to observers on Earth and the triple ring structure. Two rotation angles, $\theta$ and $\chi$, are defined as in Figure 15. For the configuration of the triple ring structure, see Sugerman et al. (2005a, 2005b) and Tziamtzis et al. (2011). The inclination angle of the ER is $\sim 43^{\circ}$ (Tziamtzis et al. 2011). Figure 16 shows normalized masses of ${ }^{56} \mathrm{Ni}$ as a function of the line-of-sight velocity (solid lines). The dependence of the distribution on the angle $\theta$, defined in Figure 15, is presented compared with the observed [Fe II] lines (points with error bars), 18 and $26 \mu \mathrm{m}$ (Haas et al. 1990). Here the rotation angle $\chi$ is fixed to be $0^{\circ}$. For the rotation angle $\theta$, considering the fact that the bulk of the [Fe II] line is redshifted (Haas et al. 1990) and the 3D distribution of the inner ejecta seems to be slightly tilted to the ER plane from the observations of $[\mathrm{Si} \mathrm{I}]+[\mathrm{Fe} \mathrm{II}]$ lines, $\theta$ is changed in the range between $90^{\circ}$ and $135^{\circ}$. The dependence on the progenitor models is also presented. For all models shown in the figure, the smaller the rotation angle $\theta$, the more concentrated around the velocity center the distributions. Compared with model b18.3, model n16.3 apparently lacks a high-velocity component $\left(\gtrsim 2000 \mathrm{~km} \mathrm{~s}^{-1}\right)$. In model b18.3, the tail $\left(\lesssim 4000 \mathrm{~km} \mathrm{~s}^{-1}\right)$ and peak $\left(\sim 1000 \mathrm{~km} \mathrm{~s}^{-1}\right)$ at the redshifted side are best reproduced for the rotation angle $\theta$ of $\gtrsim 130^{\circ}$ among the four models, although the distribution of the blueshifted side is insufficient. In models b18.3-fid, s18.0-fid, and s19.8-fid, in particular the latter two RSG models, double-peak structures are seen for the rotation angle $\theta$ of $\gtrsim 120^{\circ}$. The clear double-peak structures in the RSG models reflect the bicone-like distribution of ${ }^{56} \mathrm{Ni}$, as seen in the bottom panels in Figure 9. It is noted that in the points of the [Fe II] line of $26 \mu \mathrm{m}$, a valley around the velocity center (the bottom of the valley is only one point) is recognized, but clear double peaks as seen in the RSG models are inconsistent with the overall distributions from the observations. Figure 17 shows the dependence on the rotation angle $\chi$ (the model is fixed as b18.3-fid). The case of $\chi=0^{\circ}$ is shown in the top left panel in Figure 16. As can be seen, among the models of the cases of $\chi=0^{\circ}, 10^{\circ}$, and $20^{\circ}$, there are no distinct differences in the overall distributions, but the tail at the redshifted side is slightly better explained in the case of $\chi=10^{\circ}$ than the other two cases. In the cases of $\chi=30^{\circ}$ and $45^{\circ}$, the tails $\left(\gtrsim 1500 \mathrm{~km} \mathrm{~s}^{-1}\right)$ are apparently reduced compared with the cases of smaller $\chi$. Figure 18 shows the dependence on the parameter $\alpha \equiv v_{\text {up }} / v_{\text {down }}$. The values of $\alpha$ in models b18.3alpha1 and b18.3-alpha2 are 1.0 and 2.0, respectively. The case of $\alpha=1.5$ is shown in the top left panel of Figure 17. For the case of $\alpha=1.0$, in which there is no global asymmetry in the explosion against the equatorial plane, the distributions are symmetric against the velocity center, as expected, and symmetric double peaks are seen for the rotation angle $\theta$ of $\gtrsim 120^{\circ}$. For the case of $\alpha=2.0$, compared with the case of $\alpha=1.5$, the tail at the redshifted side is slightly enhanced, and the peak at the blueshifted side is reduced for the rotation angle $\theta$ of $\gtrsim 120^{\circ}$. In models b18.3-fid and b18.3-alpha2, the sharp cutoffs at the blueshifted side are seen at velocities around 2000 and $1500 \mathrm{~km} \mathrm{~s}^{-1}$, respectively. Then, the observed tails at the blueshifted side are more difficult to reproduce in model b18.3alpha2 than in model b18.3-fid.

Based on the results of lower-resolution simulations and arguments on the constraints from the observations of $\mathrm{SN}$ 

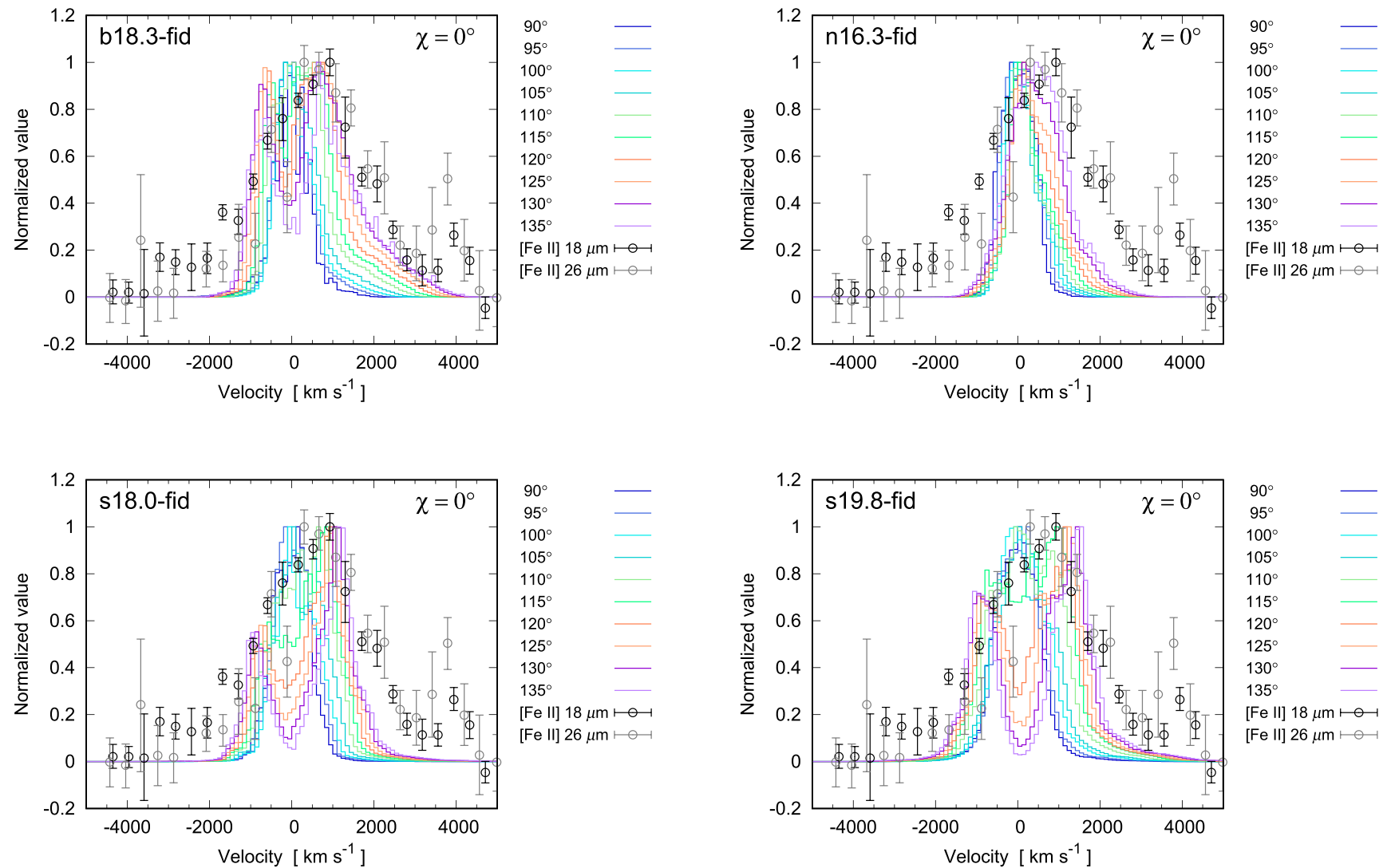

Figure 16. Normalized masses of ${ }^{56} \mathrm{Ni}$ as a function of the line-of-sight velocity at the end of the simulation. The top left, top right, bottom left, and bottom right panels are for models b18.3-fid (80,621 s), n16.3-fid (85,714 s), s18.0-fid (279,037 s), and s19.8-fid (283,161 s), respectively. The points with error bars $(1 \sigma)$ are the normalized observed fluxes of the [Fe II] lines, 18 and $26 \mu \mathrm{m}$ (Haas et al. 1990), where continuum levels are subtracted. Normalizations are carried out in order for the peak value to be unity. Each solid line is the result with an angle, $\theta$, defined in Figure 15. Here the rotation angle $\chi$ is fixed to be $0^{\circ}$.

$1987 \mathrm{~A}$ on the mass of high-velocity ${ }^{56} \mathrm{Ni}$ and the mass distributions of the line-of-sight velocity of ${ }^{56} \mathrm{Ni}$, favored values of parameters related to the asymmetric explosions and progenitor models are presented by comparing representative models. As mentioned in Section 1, the progenitor of SN 1987A, Sk $-69^{\circ} 202$, was a compact BSG at the time of the explosion. Then, models with one of the BSG pre-supernova models b18.3 and n16.3 are appropriate. From the constraints on the mass of high-velocity ${ }^{56} \mathrm{Ni}$, i.e., (i) the ratio of the mass of ${ }^{56} \mathrm{Ni}$ that has $\geqslant 3000 \mathrm{~km} \mathrm{~s}^{-1}$ to the total ${ }^{56} \mathrm{Ni}$ mass is greater than $4 \%$ and (ii) the mass of ${ }^{56} \mathrm{Ni}$ that has $\geqslant 4000 \mathrm{~km} \mathrm{~s}^{-1}$ is greater than $10^{-3} M_{\odot}$, the models with large $\beta \equiv v_{\mathrm{pol}} / v_{\mathrm{eq}}$ values (8 or 16), b18.3-beta8, b18.3-fid, b18.3-alpha2, and b18.3-clp0, are selected as candidates for SN 1987A. As mentioned before, there is no explosion model with BSG model n16.3 (single-star evolution) that satisfies the two conditions on the mass of high-velocity ${ }^{56} \mathrm{Ni}$ simultaneously. Among models b18.3-beta8, b18.3-fid, and b18.3-clp0, the mass of high-velocity ${ }^{56} \mathrm{Ni}$ in model b18.3-beta8 is a bit smaller than that in the other two models (see Table 3 ). The difference in the initial setup between models b18.3-fid and b18.3-clp0 is the existence of the fluctuations in the initial radial velocities. It is a bit arbitrary but motivated by the recent observations of $\mathrm{CO}$ and $\mathrm{SiO}$ molecules and dust in the inner ejecta of SN 1987A (Abellán et al. 2017; Cigan et al. 2019), which have indicated that the inner ejecta is clumpy (the first observational evidence of clumpiness of the ejecta of SN 1987A was found from narrow features in emission lines, e.g., [O I]; Stathakis et al. 1991); we thus prefer the model with the initial fluctuations, i.e., b18.3-fid. Finally, model b18.3-alpha2 is also a candidate, as well as model b18.3-fid. As discussed before, considering the deficiency in the tail at the blueshifted side in the mass distribution of ${ }^{56} \mathrm{Ni}$ as a function of the line-of-sight velocity, we select the model with a moderate value for the parameter $\alpha \equiv v_{\text {up }} / v_{\text {down }}=1.5$, i.e., b18.3-fid, as a fiducial model in the models of lower-resolution simulations.

\subsection{Results of 3D Simulations: High-resolution Cases}

Based on the arguments on the exploration of lower-resolution simulations in Section 4.2, two high-resolution simulations with the two BSG progenitor models b18.3 and n16.3 are performed, where the high-resolution models are denoted as b18.3-high and n16.3-high, and the corresponding lower-resolution models are b18.3-fid and n16.3-fid, respectively. The parameters for the aspherical explosion are fixed to be same as model b18.3-fid (see Table 2 for the values of the parameters). First, the results listed in Table 3 are discussed by comparing with those of the corresponding lower-resolution models. Obtained explosion energies, $E_{\text {exp }}$, of higher- (lower-) resolution models b18.3-high (b18.3-fid) and n16.3-high (n16.3-fid) are $2.01 \times 10^{51} \mathrm{erg}$ $\left(1.99 \times 10^{51} \mathrm{erg}\right)$ and $1.93 \times 10^{51} \mathrm{erg}\left(1.91 \times 10^{51} \mathrm{erg}\right)$, respectively. The values of the high-resolution models are slightly higher compared with those of the lower-resolution models, but the values are consistent enough with those of the lower-resolution models. The ejected masses of ${ }^{56} \mathrm{Ni}$ in models b18.3-high (b18.3-fid) and n16.3-high (n16.3-fid) are $8.64 \times 10^{-2} M_{\odot}$ $\left(8.10 \times 10^{-2} M_{\odot}\right)$ and $9.67 \times 10^{-2} M_{\odot}\left(9.04 \times 10^{-2} M_{\odot}\right)$, 

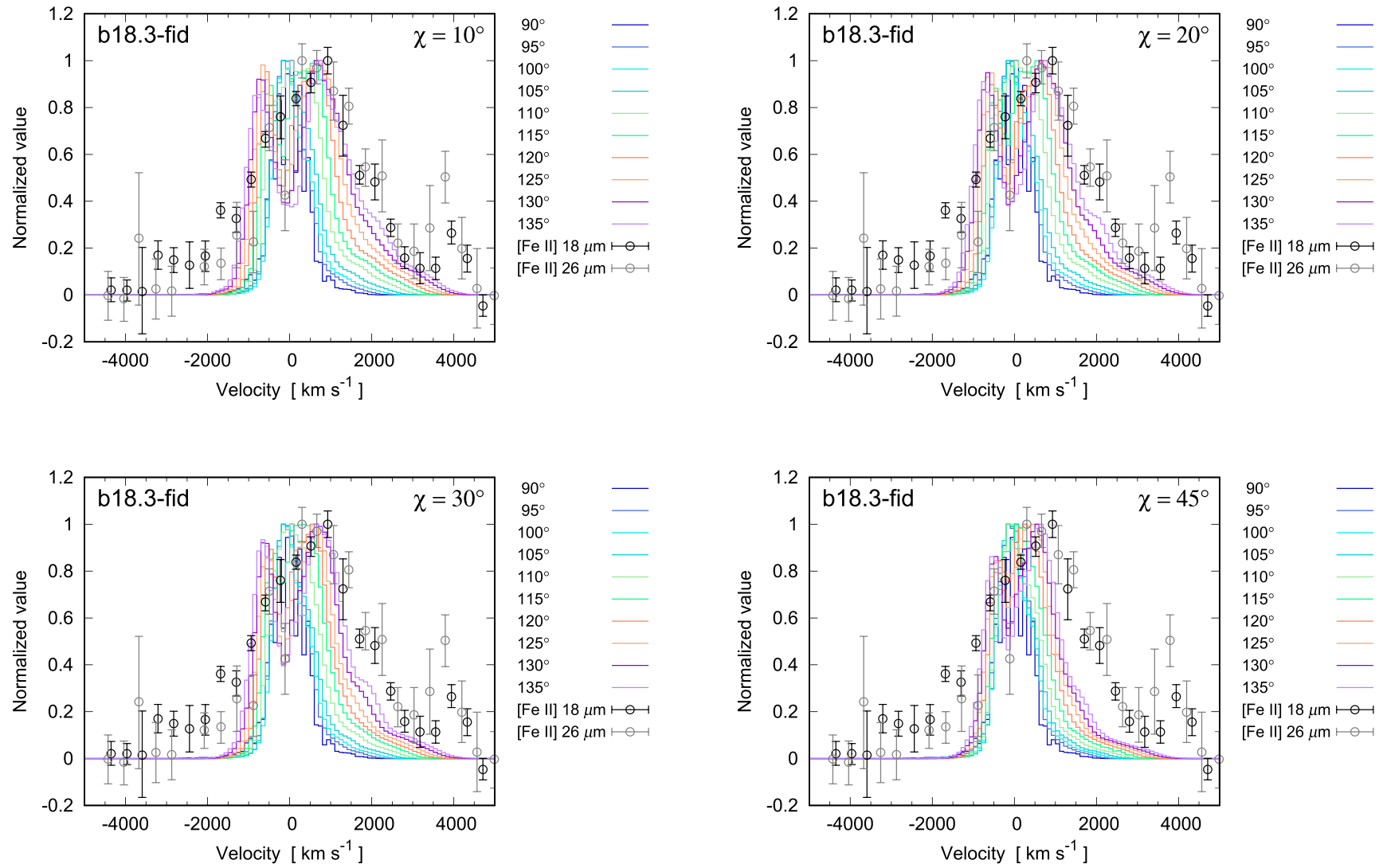

Figure 17. Same as Figure 16 but for model b18.3-fid (80,621 s) with a parameter $\chi$ of $10^{\circ}$ (top left), $20^{\circ}$ (top right), $30^{\circ}$ (bottom left), and $45^{\circ}$ (bottom right).
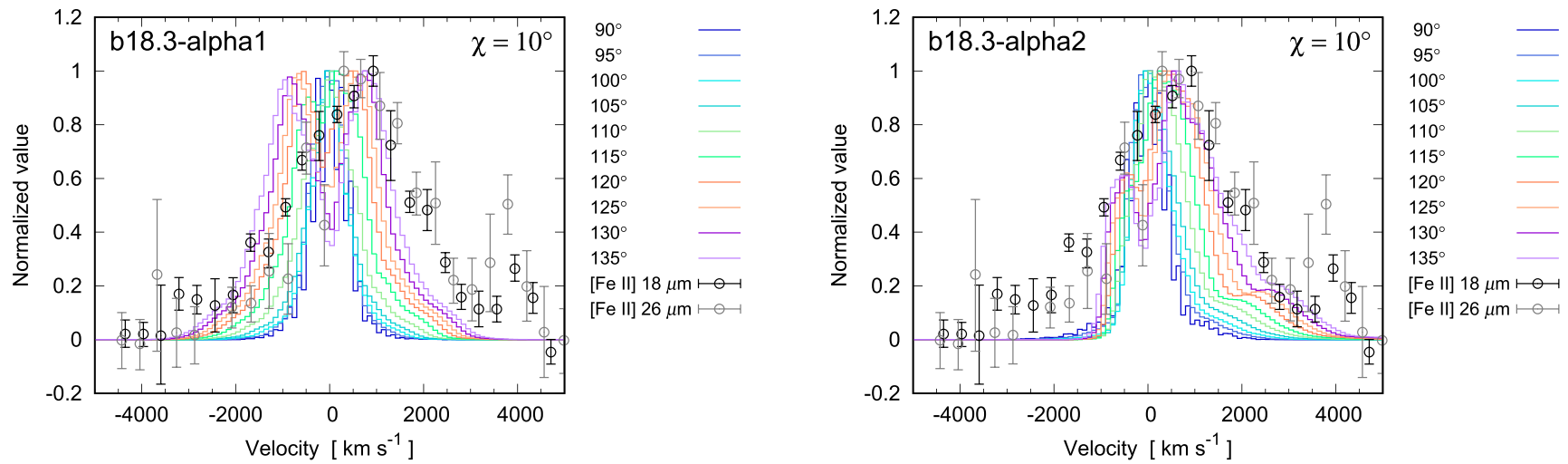

Figure 18. Same as Figure 16 but for models b18.3-alpha1 (left; 77,425 s) and b18.3-alpha2 (right; 77,261 s) and the parameter $\chi=10^{\circ}$.

respectively. The ejected masses of ${ }^{44} \mathrm{Ti}$ in models b18.3-high (b18.3-fid) and n16.3-high (n16.3-fid) are $5.73 \times 10^{-4} M_{\odot}$ $\left(7.39 \times 10^{-4} M_{\odot}\right)$ and $5.38 \times 10^{-4} M_{\odot}\left(7.17 \times 10^{-4} M_{\odot}\right)$, respectively. Therefore, the masses of ${ }^{56} \mathrm{Ni}$ in the lower-resolution models are underestimated by $\sim 5 \%$ compared with those of the high-resolution models. On the other hand, the masses of ${ }^{44} \mathrm{Ti}$ in the lower-resolution models are overestimated by 20\%-30\% compared with those of the high-resolution models. As mentioned in Section 4.2, the nuclear reaction network in the simulations in this paper includes only 19 nuclei, and the mass fractions of ${ }^{56} \mathrm{Ni}$ and ${ }^{44} \mathrm{Ti}$ could be overestimated by factors of $\sim 1.5$ and 3 , respectively, compared with those calculated with a larger nuclear reaction network. If we correct for the overestimation, the masses of ${ }^{56} \mathrm{Ni}$ in models b18.3-high and n16.3-high could be $\lesssim 0.06 M_{\odot}$, which is roughly consistent with the value suggested by the observations, $0.07 M_{\odot}$ (e.g., Shigeyama \& Nomoto 1990). The masses of ${ }^{44} \mathrm{Ti}$ in models b18.3-high and n16.3-high could be $\lesssim 2 \times 10^{-4} M_{\odot}$, which is also consistent with the values deduced from the observations, $(3.1 \pm 0.8) \times 10^{-4}$ and $(1.5 \pm 0.3) \times$ $10^{-4} M_{\odot}$ (Grebenev et al. 2012; Boggs et al. 2015, respectively).

The values related to the observational constraints on the mass of high-velocity ${ }^{56} \mathrm{Ni}$ (see Table 3 and Figure 11), i.e., $M_{4.0}\left({ }^{56} \mathrm{Ni}\right)$ and $M_{3.0} / M_{\mathrm{ej}}\left({ }^{56} \mathrm{Ni}\right)$, in model b18.3-high (b18.3fid) are $1.11 \times 10^{-3} M_{\odot}\left(1.89 \times 10^{-3} M_{\odot}\right)$ and $1.05 \times 10^{-1}$ $\left(1.38 \times 10^{-1}\right)$, respectively. The values of $M_{4.0}\left({ }^{56} \mathrm{Ni}\right)$ and $M_{3.0} / M_{\mathrm{ej}}\left({ }^{56} \mathrm{Ni}\right)$ in model n16.3-high (n16.3-fid) are 

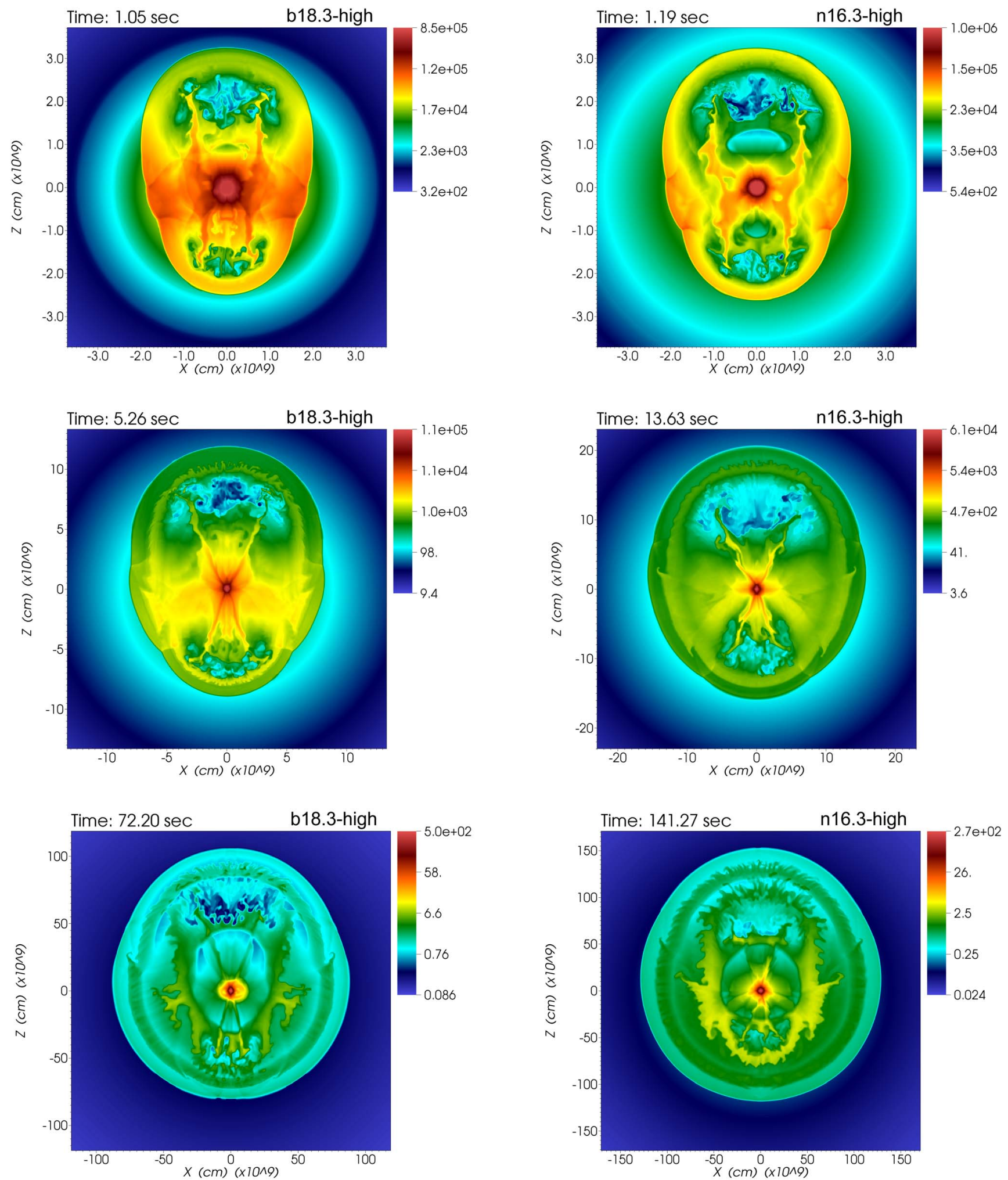

Figure 19. Density color maps (2D slices in the $x-z$ plane) in a logarithmic scale. The unit of the values in the color bars is $\mathrm{g} \mathrm{cm}^{-3}$. Left (right) panels are for model b18.3-high (n16.3-high). The top, middle, and bottom panels map the times when the blast wave is inside the C+O, helium, and hydrogen layers, respectively. An animation (density color maps over time) for this figure and Figure 20 is available. In the animation embedded in this figure, snapshots only for model b18.3-high (left panels) are shown. The video starts at $t=0 \mathrm{~s}$ and ends at $t=68,357.48 \mathrm{~s}$. The real-time duration of the video is $16 \mathrm{~s}$.

(An animation of this figure is available.)

$2.50 \times 10^{-4} M_{\odot}\left(6.08 \times 10^{-5} M_{\odot}\right)$ and $3.71 \times 10^{-2}(1.40 \times$ $10^{-2}$ ), respectively. Then, the values in model b18.3-fid tend to be overestimated compared with those of model b18.3-high.
On the other hand, the values in model n16.3-fid tend to be underestimated compared with those of model n16.3-high. The opposite responses to the increase in resolution of the 

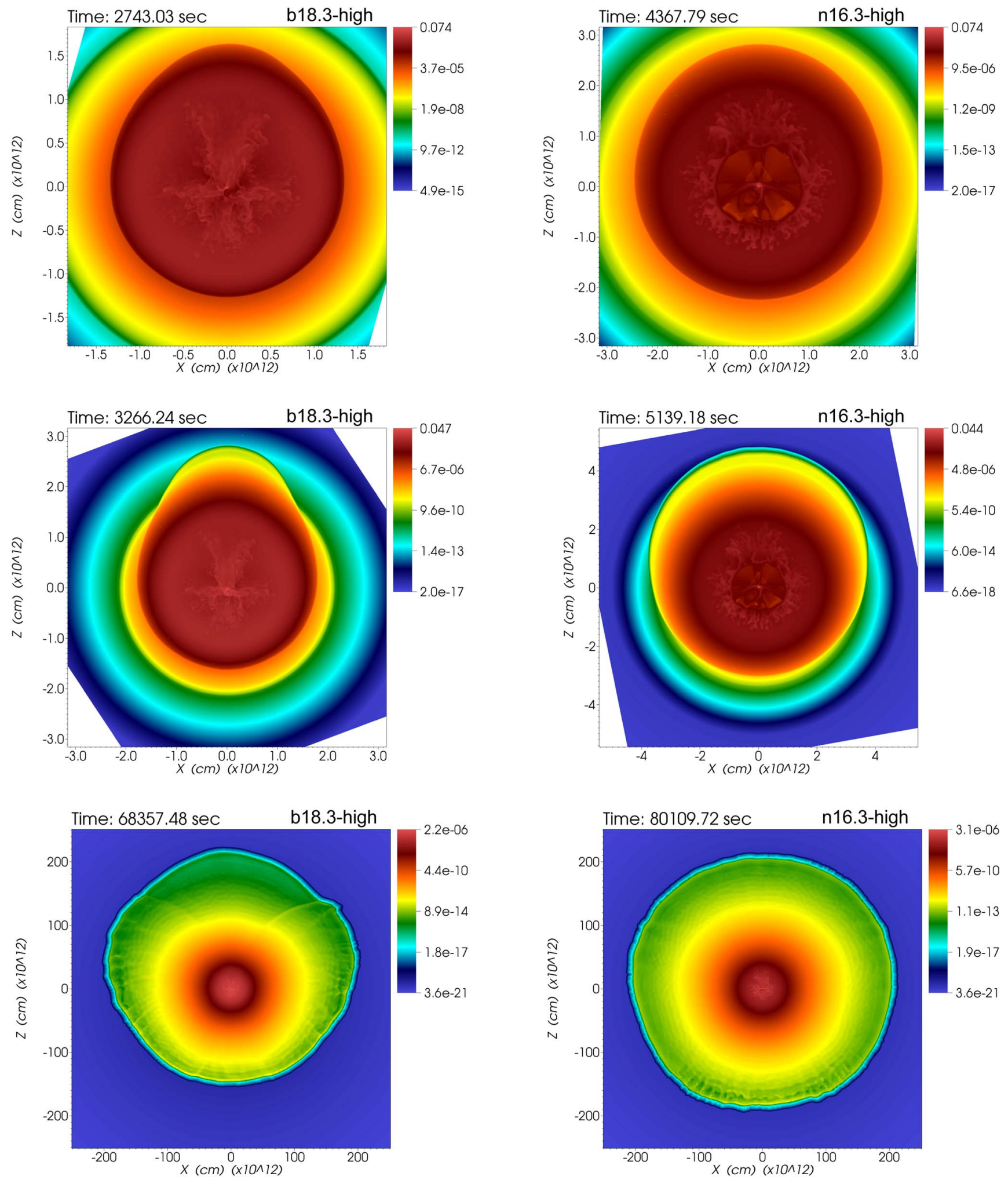

Figure 20. Density color maps (2D slices in the $x-z$ plane) in a logarithmic scale. The unit of the values in the color bars is $\mathrm{g} \mathrm{cm}^{-3}$. Left (right) panels are for model b18.3-high (n16.3-high). The top, middle, and bottom panels map the times just before the shock breakout, just after the shock breakout, and the end of the simulation, respectively. An animation (density color maps over time) for this figure and Figure 20 is available. In the animation embedded in this figure, snapshots only for model n16.3-high (right panels) are shown. The video starts at $t=0 \mathrm{~s}$ and ends at $t=80,109.72 \mathrm{~s}$. The real-time duration of the video is $16 \mathrm{~s}$.

(An animation of this figure is available.)

simulations between the two progenitor models may be attributed to the difference of the significance of $\mathrm{RT}$ instabilities. Since progenitor model n16.3 has larger $\mathrm{C}+\mathrm{O}$ and helium cores with steep gradients in the $\rho r^{3}$ profile, RT instability may play a more significant role in order to convey the innermost ${ }^{56} \mathrm{Ni}$ into outer high-velocity layers 
than that in model b18.3. In model n16.3-high, by capturing smaller-scale perturbations, the growth of RT instabilities could be faster than in model n16.3-fid. Hence, the mass of highvelocity ${ }^{56} \mathrm{Ni}$ in model n16.3-high could be largely due to the efficient growth of the instabilities compared with that in model $\mathrm{n} 16.3$-fid. While the role of RT instabilities is less important in model b18.3, the situation could be opposite to model n16.3. Although the obtained masses of the high-velocity ${ }^{56} \mathrm{Ni}$ are slightly different from those of lower-resolution models, it is not changed between the high- and lower-resolution models whether the model satisfies the observational constraints or not. Model b18.3-high satisfies the two conditions, i.e., (i) the ratio of the mass of ${ }^{56} \mathrm{Ni}$ that has $\geqslant 3000 \mathrm{~km} \mathrm{~s}^{-1}$ to the total ${ }^{56} \mathrm{Ni}$ mass is greater than $4 \%$ and (ii) the mass of ${ }^{56} \mathrm{Ni}$ that has $\geqslant 4000 \mathrm{~km} \mathrm{~s}^{-1}$ is greater than $10^{-3} M_{\odot}$. On the other hand, n16.3-high fails to satisfy the two conditions.

Figures 19 and 20 show the time evolution of the density distributions for models b18.3-high (left) and n16.3-high (right). The distributions at the early phase $(\sim 1 \mathrm{~s}$; top panels of Figure 19) can be compared with the corresponding density distributions of lower-resolution simulations in the top panels of Figure 7. At that time, the blast wave $\left(r \sim 3 \times 10^{9} \mathrm{~cm}\right)$ is inside the $\mathrm{C}+\mathrm{O}$ layer. The shape of the blast wave is almost the same as in the corresponding lower-resolution simulation. As seen in Figures 7 and 19, in the high-resolution models, smaller-scale structures due to instabilities are more pronounced than those in the lower-resolution models. As is the case with the lower-resolution simulations, the bipolar structure in model n16.3-high is wider than that of b18.3-high due to stronger deceleration inside the $\mathrm{C}+\mathrm{O}$ layer with a steeper gradient in the $\rho r^{3}$ profile of the progenitor model (see Figures 1 and 2) than in the case of b18.3-high. Inside the bipolar structure, fingers due to RT and/or Kelvin-Helmholtz instabilities develop along the bipolar axis. After the blast wave goes through the $\mathrm{C}+\mathrm{O} / \mathrm{He}$ interface, fingers of RT instability start to grow on top of the reverse shock developed by the deceleration during the shock propagation inside the helium layer (see middle panels in Figure 19). The radii of the composition interface of the $\mathrm{C}+\mathrm{O} / \mathrm{He}$ of progenitor models b18.3 and n16.3 are $3.5 \times 10^{9}$ and $6.1 \times 10^{9} \mathrm{~cm}$, respectively. Part of the tips (terminal ends) of the fingers developed at an early phase (fingers seen in the top panels) seems to touch the reverse shock. After the blast wave passes the $\mathrm{He} / \mathrm{H}$ interface, another reverse shock develops outside the previous one caused by the strong deceleration during the shock propagation inside the hydrogen layer (see bottom panels in Figure 19). On top of the newly developed (outer) reverse shock, fingers of RT instability start to grow. Then, a nested double shell structure with fingers develops. The radii of the $\mathrm{He} / \mathrm{H}$ composition interface in models b18.3 and n16.3 are $2.9 \times 10^{10}$ and $5.2 \times 10^{10} \mathrm{~cm}$, respectively. It is noted that RT fingers start to grow from the inner shell not only along the polar direction but also near the equatorial plane, where denser material (yellow) exists than in polar regions inside the inner shell. Before the shock breakout, the inner shell (inner reverse shock) with RT fingers is swept up by the outer inward shell (reverse shock) during the propagation of the blast wave into the hydrogen envelope. Just before the shock breakout, the outer reverse shock has swept up almost all inner ejecta in model b18.3-high (see the top left panel in Figure 20). On the other hand, in model n16.3-high, the last formed reverse shock is still propagating inward, even after the shock breakout (see the top and middle right panels in Figure 20). In the top right panel (just before the shock breakout), the reverse shock is around $r$ $\sim 1 \times 10^{12} \mathrm{~cm}$. After the shock breakout (middle panels in Figure 20), the blast wave is accelerating due to the steep pressure gradient around the original stellar surface, leaving the inner ejecta far behind. Here the radii of the stellar surface in models b18.3 and n16.3 are $2.1 \times 10^{12}$ and $3.4 \times 10^{12} \mathrm{~cm}$, respectively. Depending on the density and pressure gradients around the stellar surface, the shock breakout in model b18.3high takes place in a more aspherical way than in model $\mathrm{n} 16.3$ high (middle panels in Figure 20). The times of the shock breakout in models b18.3-high and n16.3-high are $~ 3000$ and $\sim 5000 \mathrm{~s}$, respectively, which should reflect the acceleration/ deceleration during the shock propagation, depending on the density structure of the progenitor model, but in the end, it is roughly proportional to the stellar radius. Finally, at the end of the simulation (bottom panels in Figure 20), the inner ejecta consisting of the material originally inside the helium core is far behind the blast wave. The shape of the blast wave in model b18.3-high is more aspherical than that in model n16.3-high. Figures 21 and 22 show the time evolution of the ${ }^{56} \mathrm{Ni}$ distribution for models b18.3-high (left) and n16.3-high (right), which correspond to the time evolutions of the density distribution in Figures 19 and 20, respectively. The early ( $\sim 1 \mathrm{~s})$ distributions of ${ }^{56} \mathrm{Ni}$ in the high-resolution models can be compared with the corresponding lower-resolution models, b18.3-fid and n16.3-fid (top panels in Figure 8). Although smaller-scale structures are seen in models b18.3-high and n16.3-high than in the lower-resolution models, the overall distributions of ${ }^{56} \mathrm{Ni}$ are the same as in the lower-resolution models. At the early phase, ${ }^{56} \mathrm{Ni}$ exists inside the fingers on the tips of the bipolar structure (top panels in Figure 21). During the propagation of the blast wave into the helium layer (middle panels of Figure 21), part of the ${ }^{56} \mathrm{Ni}$ falls back into the compact object along the equatorial plane, and the equatorial regions of the bipolar distribution are shrunken. At this phase, the tips of the bipolar distribution of ${ }^{56} \mathrm{Ni}$ reach the shell (reverse shock) with RT fingers seen in the corresponding density distribution (middle panels in Figure 19). After the blast wave passes the $\mathrm{He} / \mathrm{H}$ interface, as mentioned before, the nested double shell structure forms (bottom panels in Figure 19). An interesting difference between the two progenitor models is whether the tips of the ${ }^{56} \mathrm{Ni}$ distribution reach the outer shell (the newly formed reverse shock during the shock propagation into the hydrogen layer) or not. In model b18.3-high, the tips of ${ }^{56} \mathrm{Ni}$ penetrate the inner shell and touch the outer shell (see bottom left panels in Figures 19 and 21). On the other hand, in model n16.3-high, ${ }^{56} \mathrm{Ni}$ remains confined to inside the inner shell (bottom right panels in Figures 19 and 21). As seen in the top two rows in Figure 3 in Section 4.1, for both progenitor models, instabilities grow around the composition interfaces of the $\mathrm{C}+\mathrm{O} / \mathrm{He}$ and $\mathrm{He} / \mathrm{H}$. Then, the inner and outer shells (bottom panels in Figure 19) can be approximately regarded as the $\mathrm{C}+\mathrm{O} / \mathrm{He}$ and $\mathrm{He} / \mathrm{H}$ composition interfaces, respectively. Hence, the bulk of the ${ }^{56} \mathrm{Ni}$ in model n16.3-high is confined to the $\mathrm{C}+\mathrm{O}$ layer, whereas part of the ${ }^{56} \mathrm{Ni}$ in model b18.3-high penetrates the helium layer to reach the hydrogen layer. After the inward outer shell sweeps up the inner shell, in model n16.3-high, part of the ${ }^{56} \mathrm{Ni}$ penetrates into the outer high-velocity layers consisting of helium and hydrogen, but the bulk of the ${ }^{56} \mathrm{Ni}$ remains confined to the helium core (top right panels in Figures 20 and 22). In model b18.3-high, part of the 

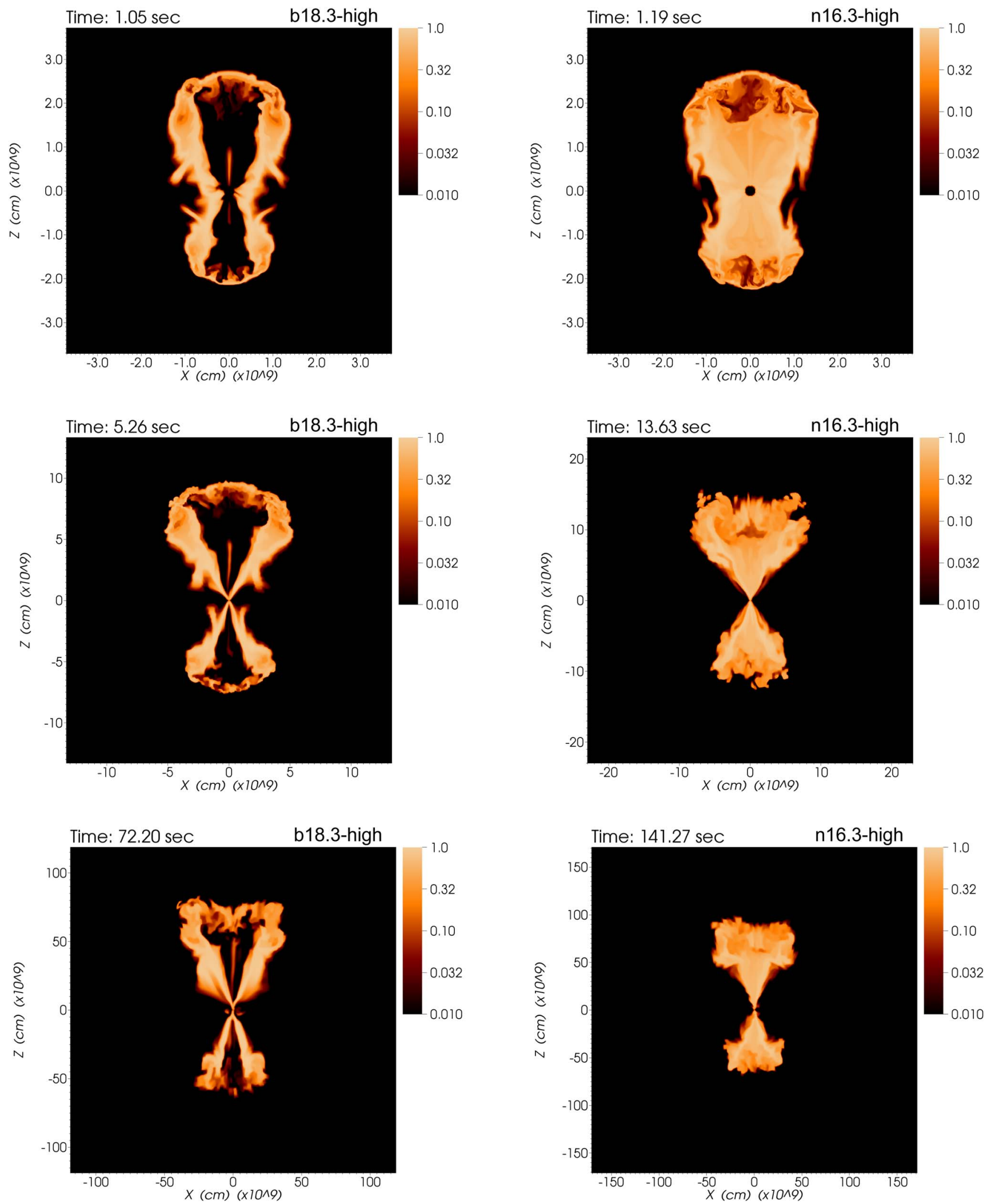

Figure 21. Same as Figure 20 but for color maps of the mass fraction of ${ }^{56} \mathrm{Ni}$.

${ }^{56} \mathrm{Ni}$ reaches the tips of the extended RT fingers (top left panels in Figures 20 and 22). After the shock breakout (middle and bottom panels in Figure 22), the inner ejecta, including ${ }^{56} \mathrm{Ni}$, is left far behind the blast wave.
Figure 23 shows the distributions of elements ${ }^{56} \mathrm{Ni},{ }^{28} \mathrm{Si}$, ${ }^{16} \mathrm{O}$, and ${ }^{4} \mathrm{He}$ just before the shock breakout for models b18.3high (left) and n16.3-high (right). Compared with the distributions in the corresponding lower-resolution models 

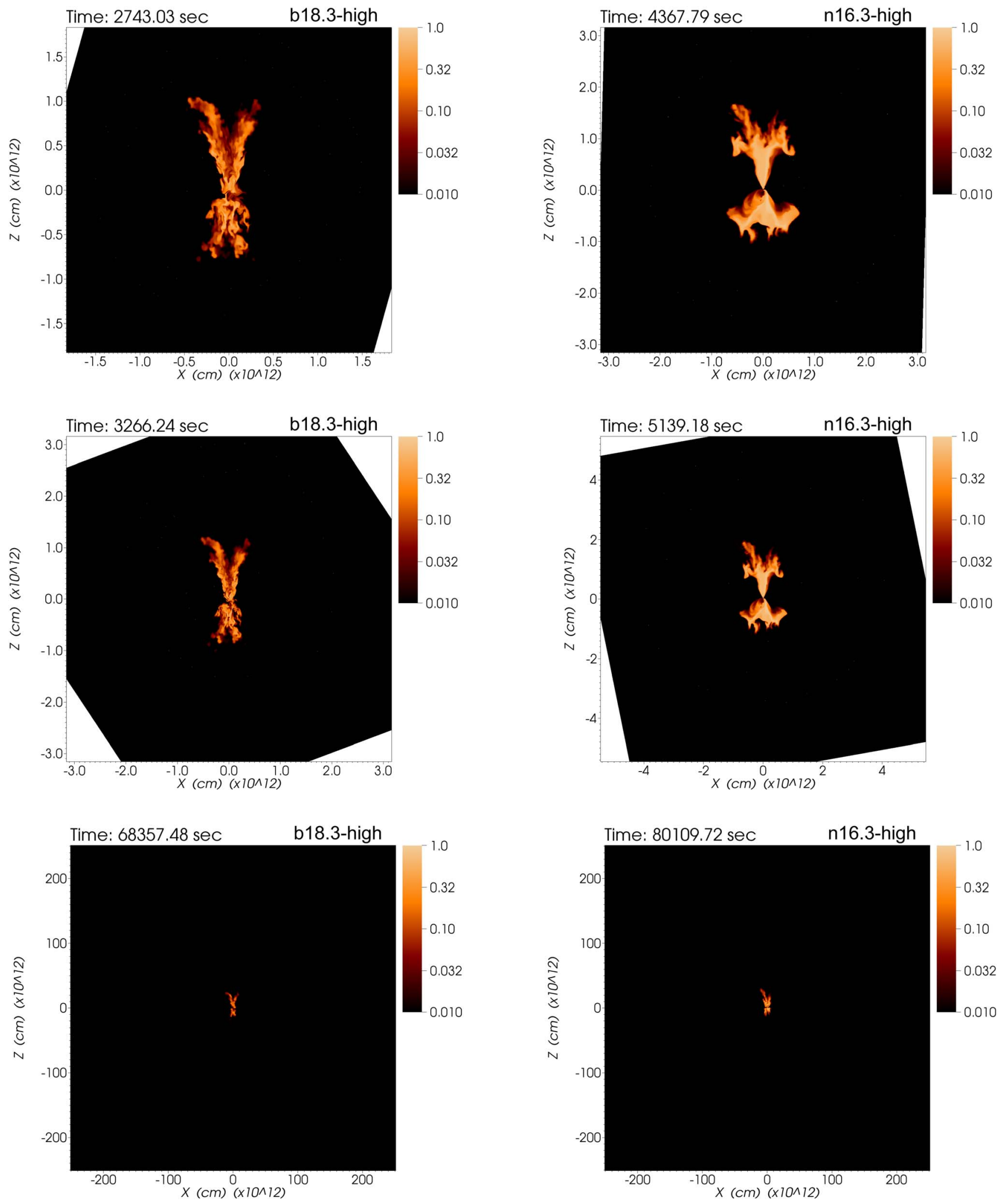

Figure 22. Same as Figure 20 but for color maps of the mass fraction of ${ }^{56} \mathrm{Ni}$.

(top panels in Figure 10), the global morphologies of the distributions are consistent with the lower-resolution models, although smaller-scale structures are resolved. In model b18.3high, the fingers are extended from the central region, which reflects the fact that the reverse shock developed during the shock propagation into the hydrogen envelope has already swept up the inner ejecta before the shock breakout. In model $\mathrm{n} 16.3$-high, the reverse shock is still propagating into the inner ejecta, even after the shock breakout and the fingers consisting of ${ }^{16} \mathrm{O}$ and ${ }^{4} \mathrm{He}$ are extended from the reverse shock surface. Then, a diluted space inside the shell with the ${ }^{16} \mathrm{O}$ and ${ }^{4} \mathrm{He}$ fingers is visible in model n16.3-high (right panel). As 

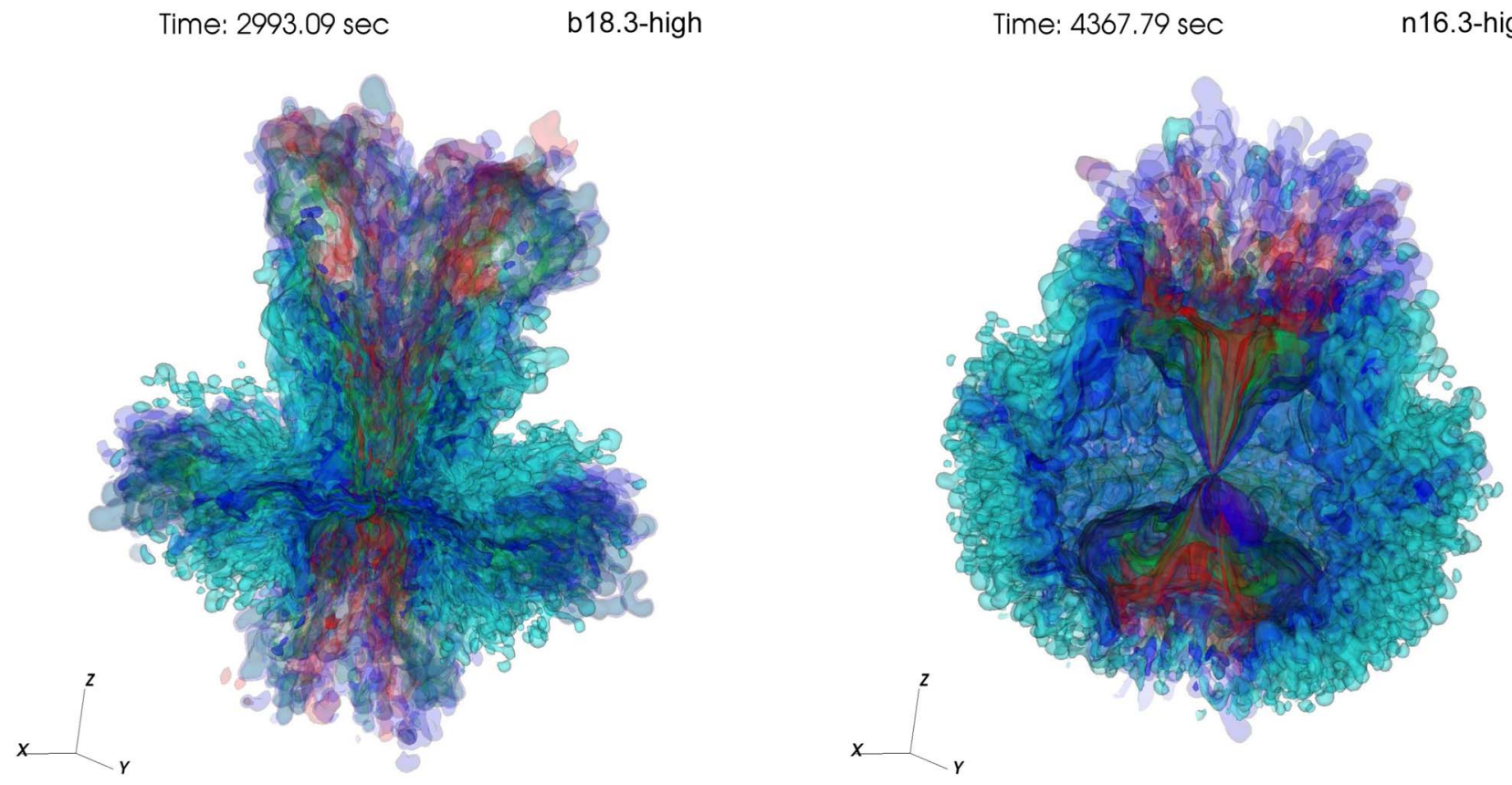

Figure 23. Same as Figure 10 but for models b18.3-high (left) and n16.3-high (right). An animation (distributions from different viewing angles and ones with and without clipping) for this figure is available. The real-time duration of the video is $12 \mathrm{~s}$. Interactive 3D models on Sketchfab corresponding to left (https://skfb.ly/ 6OZDu) and right (https://skfb.ly/6OZDv) panels are also available.

(An animation of this figure is available.)
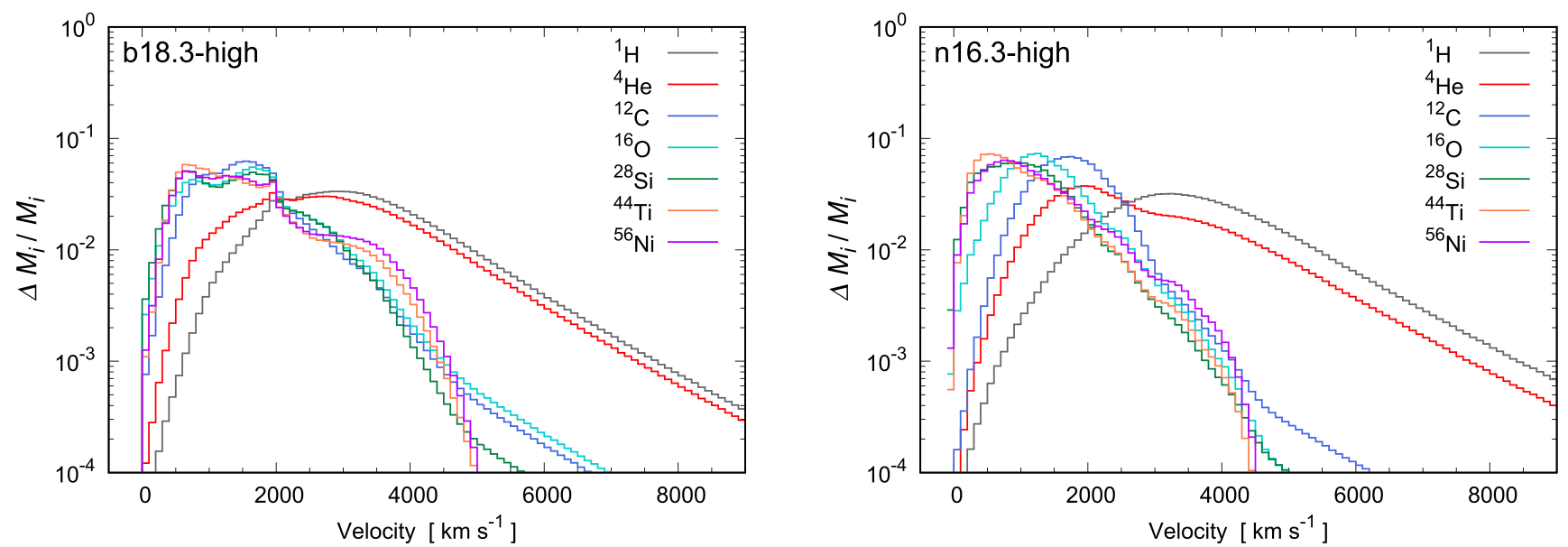

Figure 24. Same as Figure 14 but for models b18.3-high (left; 68,357 s) and n16.3-high (right; 80,110 s).

mentioned above, in model n16.3-high, a small fraction of ${ }^{56} \mathrm{Ni}$ penetrates into the extended fingers along the polar direction, but the bulk of the ${ }^{56} \mathrm{Ni}$ is confined to the helium core. In model b18.3-high, the penetration of ${ }^{56} \mathrm{Ni}$ into the tips of the extended fingers is observed.

Figure 24 shows the distributions of the radial velocity of ${ }^{56} \mathrm{Ni}$ for models b18.3-high (left) and n16.3-high (right). The distributions can be compared with the lower-resolution models b18.3-fid and n16.3-fid (top panels in Figure 14). Compared with the lower-resolution model b18.3-fid, the high-velocity ${ }^{56} \mathrm{Ni}$ is slightly reduced in model b18.3-high. A bump seen at around $\sim 2000-2500 \mathrm{~km} \mathrm{~s}^{-1}$ in b18.3-fid (Figure 14) is flattened in model b18.3-high, and instead, lower-velocity ${ }^{56} \mathrm{Ni}\left(\sim 2000 \mathrm{~km} \mathrm{~s}^{-1}\right)$ is slightly enhanced. On the other hand, compared with the lower-resolution model n16.3-fid, in model n16.3-high, ${ }^{56} \mathrm{Ni}$ of a velocity around 2500 and $3500 \mathrm{~km} \mathrm{~s}^{-1}$ is slightly enhanced. As mentioned in Section 4.2, opposite responses to the increase in resolution of the simulations are seen, which may be attributed to the difference of the significance of the RT instability between the two progenitor models. Although there are slight differences between the lower- and high-resolution models, the superiority of model b18.3 in terms of the amount of high-velocity ${ }^{56} \mathrm{Ni}$ is not changed between the lower- and high-resolution models. The mass of the high-velocity ${ }^{56} \mathrm{Ni}\left(\gtrsim 3000 \mathrm{~km} \mathrm{~s}^{-1}\right)$ in model b18.3high is larger than that of model n16.3-high by a factor of $\sim 3$ (see also Table 3).

Figure 25 shows the distributions of the line-of-sight velocity of ${ }^{56} \mathrm{Ni}$ for models b18.3-high (left) and n16.3-high (right). Here, based on the discussion on the rotation angle $\chi$ in Section 4.2, the value of $\chi$ is set to be $10^{\circ}$. The distribution in b18.3-high can be directly compared with the corresponding 

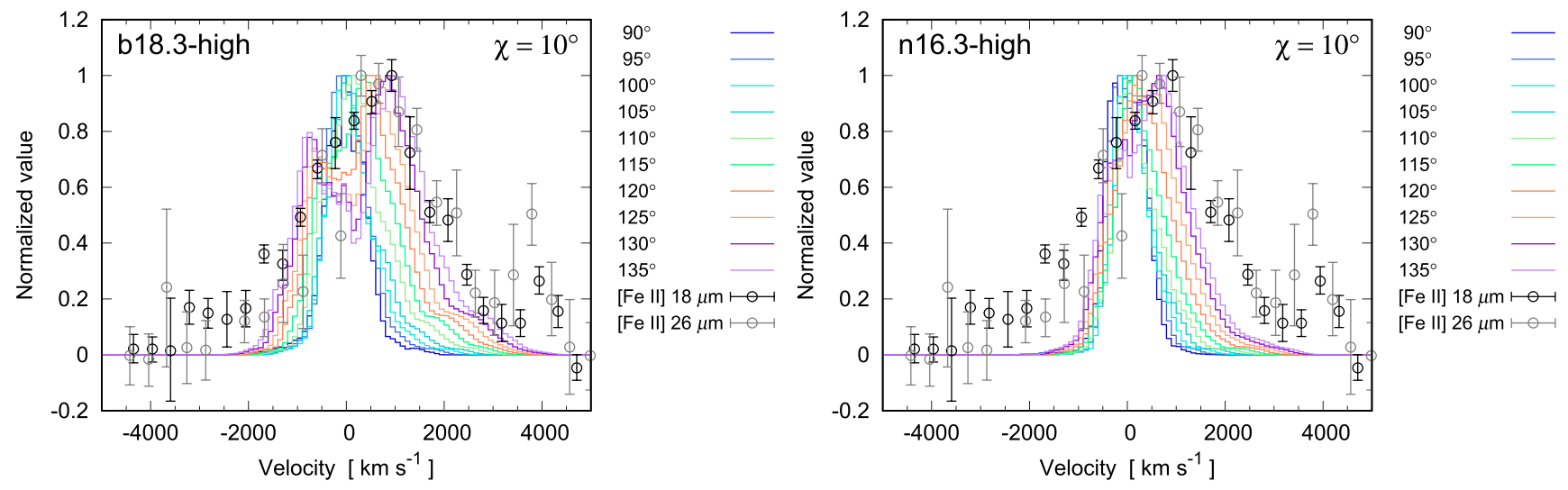

Figure 25. Same as Figure 16 but for models b18.3-high (left; 68,357 s) and n16.3-high (right; 80,110 s) and the parameter $\chi=10^{\circ}$.

model b18.3-fid with the same value for $\chi$ (top left panel in Figure 17). Compared with the distribution in model b18.3-fid, the one of the double peaks for the $\theta \gtrsim 120^{\circ}$ at the blueshifted side around $-700 \mathrm{~km} \mathrm{~s}^{-1}$ is reduced, and the value of the peak approaches the values of nearby observed points. The tail around $-2000 \mathrm{~km} \mathrm{~s}^{-1}$ is slightly enhanced, and the tail around $2000 \mathrm{~km} \mathrm{~s}^{-1}$ is slightly reduced. The distribution in model n16.3-high can be compared with that in model n16.3-fid with $\chi=0^{\circ}$ (top right panel in Figure 16). Although the value of $\chi$ in Figure $25\left(\chi=10^{\circ}\right)$ is different from the one in Figure 16 $\left(\chi=0^{\circ}\right)$, the distribution in $\mathrm{n} 16.3$-fid with $\chi=10^{\circ}$ is very similar to the case of $\chi=0^{\circ}$. Compared with the distribution in model n16.3-fid with $\chi=0^{\circ}$, the tails around -1000 and $1500 \mathrm{~km} \mathrm{~s}^{-1}$ are slightly enhanced. As a summary, the distribution in model b18.3-high better reproduces the highvelocity tails of the observed fluxes of [Fe II] lines, in particular the tail at the redshifted side, than that in model n16.3-high. As for the rotation angle $\theta$, the value of $\theta=130^{\circ}-135^{\circ}$ is preferred to fit the observed points. Motivated by the observed 3D distributions of the inner ejecta of SN 1987A (see, e.g., Figure 11 in Larsson et al. 2016; the ejecta of the redshifted side seems to be closer to us than the ER plane), a smaller $\theta$ value may be preferred. For the value of $\chi, \chi=0^{\circ}-20^{\circ}$ is a possible range, as discussed in Section 4.2 but in the case of $\chi=10^{\circ}$, the distribution better explains the observed fluxes. Therefore, we propose the parameter set of $(\theta, \chi)=\left(130^{\circ}, 10^{\circ}\right)$ as fiducial values for the rotation angles.

\section{Discussion}

In this section, based on the results presented in Section 4, several related topics are discussed in more detail.

\subsection{Key Properties of the Progenitor Models and Their Impact on Matter Mixing}

As presented in Section 4 (see, e.g., Table 3), the matter mixing, in particular how the innermost ${ }^{56} \mathrm{Ni}$ can be conveyed into outer high-velocity layers consisting of helium and hydrogen, depends on the pre-supernova model. As discussed in Sections 4.2 and 4.3, among the investigated models in this paper, there is no explosion model with the BSG model n16.3 that satisfies the two observational constraints on the mass of high-velocity ${ }^{56} \mathrm{Ni}$ simultaneously. On the other hand, with BSG model b18.3 (binary merger model; Urushibata et al. 2018) and two other RSG models, s18.0 and s19.8 (Woosley et al. 2002; Sukhbold et al. 2016), we found several explosion models that satisfy the constraints. Thus, the important questions are which properties of the pre-supernova models are essential to the matter mixing and why the properties have an advantage in the matter mixing. As presented by Wongwathanarat et al. (2015), once the blast wave enters the dense helium layer, the reverse shock developed due to the strong deceleration prohibits the inner ejecta from penetrating the helium layer to reach the composition interface of $\mathrm{He} / \mathrm{H}$ where RT instability becomes active. If the inner ejecta consisting of ${ }^{56} \mathrm{Ni}$ can successfully penetrate the helium layer before the development of the reverse shock, ${ }^{56} \mathrm{Ni}$ can be conveyed into the high-velocity hydrogen layer with the help of the RT instability. Actually, high-velocity ${ }^{56} \mathrm{Ni}\left(\sim 3500 \mathrm{~km} \mathrm{~s}^{-1}\right)$ is obtained with BSG model B15 (Utrobin et al. 2015, 2019; Wongwathanarat et al. 2015). As presented in Section 4.3, in model b18.3-high in this paper, such successful penetration of ${ }^{56} \mathrm{Ni}$ is also demonstrated. On the other hand, in model n16.3high, ${ }^{56} \mathrm{Ni}$ fails to penetrate the helium layer before the development of the reverse shock. In Wongwathanarat et al. (2015), the same pre-supernova model N20 (n16.3 in this paper) with a more realistic explosion model based on the neutrino heating also fails to reproduce the high-velocity ${ }^{56} \mathrm{Ni}$. A distinct property difference between the successful BSG models b18.3 and B15 and the unsuccessful model n16.3 (N20) is the helium core mass, $M_{\mathrm{He}, \mathrm{c}}$. The masses of the helium core of models b18.3 and n16.3 are $\sim 4$ and $\sim 6 M_{\odot}$, respectively (see Table 2). The helium core mass of model B15 is $4.05 M_{\odot}$, which is very similar to the value of model b18.3. In terms of the ratio of the helium core mass to the stellar mass, $q \equiv M_{\mathrm{He}, \mathrm{c}} / M$, such a difference between the successful and unsuccessful models is also recognized. The $q$ values of models b18.3 and B15 are 0.22 and 0.27 , respectively, whereas the $q$ value of model n16.3 (N20) is 0.37 . The small masses of the helium core of models b18.3 and B15 may enable the inner ejecta to more easily penetrate the helium core before the development of the reverse shock than the case of model n16.3. On the other hand, as we observed in model n16.3, the matter mixing in other BSG models with a larger helium core mass of $\gtrsim 5 M_{\odot}$ (Utrobin et al. 2015, 2019; Wongwathanarat et al. 2015) has revealed that the maximum velocities of ${ }^{56} \mathrm{Ni}$ are insufficient to explain the observations of SN 1987A. It is worth noting that in several explosion models with the two RSG models, high-velocity ${ }^{56} \mathrm{Ni}$ is also obtained, despite the large helium core masses ( $q$ values). In the case of the two RSG models, the gradient of the $\rho r^{3}$ profile in the helium layer is overall negative, in contrast to that of model b18.3 (see 
Figures 1 and 2), and the hydrogen envelope is very extended $\left(r \sim 10^{13} \mathrm{~cm}\right)$ compared with those of b18.3 and B15 $(r \sim$ $10^{12} \mathrm{~cm}$ ). Then, during the propagation of the blast wave in the helium layer, the development of a distinct reverse shock is restricted, and the inner ejecta can reach around the $\mathrm{He} / \mathrm{H}$ interface without the deceleration by the reverse shock. Additionally, thanks to the extended hydrogen envelope, there is enough time for RT instabilities to grow, as seen in the bottom two rows in Figure 3 (see the highly developed peaks around the composition interfaces of $\mathrm{He} / \mathrm{H}$ ), which enables the inner ejecta to be mixed up into the high-velocity hydrogen layer. In this way, despite the large helium core mass, highvelocity ${ }^{56} \mathrm{Ni}$ is obtained in the RSG models.

The BSG model B15 (Woosley et al. 1988) is one of the successful models for the high-velocity ${ }^{56} \mathrm{Ni}$; however, the helium core mass, $\sim 4 M_{\odot}$, is less than the suggested value (6 $\pm 1 M_{\odot}$; Arnett et al. 1989b) from the observed luminosity of $\mathrm{Sk}-69^{\circ} 202$. The helium core mass of model n16.3 (N20), $\sim 6 M_{\odot}$, is appropriate in terms of the luminosity of $\mathrm{Sk}-69^{\circ}$ 202 , but the model is made by artificially combining an evolved helium core with a hydrogen envelope obtained from an independent stellar evolution calculation. Additionally, as we have seen above, the matter mixing in model n16.3 fails to obtain the high-velocity ${ }^{56} \mathrm{Ni}$. As mentioned in Sections 1 and 2.2, for both BSG models based on the single-star evolution scenario, several assumptions-reduced metallicity, restricted convection, enhanced mass loss, and enhancement of the helium abundance in the hydrogen envelope (the first two are for model B15, and the latter two are for the envelope of model n16.3)-have been implemented to obtain the red-to-blue evolution for $\mathrm{Sk}-69^{\circ} 202$. Thus, the BSG progenitor models based on the single-star evolution have both pros and cons. On the other hand, binary merger model b18.3 (Urushibata et al. 2018), which satisfies all observational constraints, including the luminosity of $\mathrm{Sk}-69^{\circ} 202$, has a smaller helium core mass ( $q$ value) than those of models based on the single-star evolution that satisfy the luminosity of $\mathrm{Sk}-69^{\circ} 202$, which may reflect the nature of merging processes, the penetration of the secondary into the envelope of the primary, and the dredgeup of the primary's core material into the envelope. Actually, the other recent binary merger models (Menon \& Heger 2017) that satisfy the observational constraints all have a small helium core mass of $\sim 3-4 M_{\odot}(q \sim 0.1-0.2$; see Table 4 in Menon \& Heger 2017). As a summary, from both aspects of the matter mixing and the observational constraints on $\mathrm{Sk}-69^{\circ} 202$, the binary merger scenario is preferred for SN 1987A.

\subsection{Morphology of the Supernova Ejecta and the Explosion Mechanism of SN 1987A}

As mentioned in Section 1, the 3D morphology of the inner ejecta of SN 1987A is globally elliptical/elongated (Kjær et al. 2010; Larsson et al. 2013, 2016). The distributions of the observed [Fe II] lines in the Doppler velocity are biased toward the redshifted side. Motivated by the observations, in this paper, bipolar-like explosions with asymmetry against the equatorial plane are explored. Theoretically, the shock revival of a canonical core-collapse supernova explosion could be triggered by neutrino heating aided by SASI and/or convection, and such asymmetric bipolar explosions could be realized if a low unstable mode $(l=1)$ of SASI is dominating, as seen in 2D hydrodynamical simulations (e.g., Scheck et al. 2006; Suwa et al. 2010; Pan et al. 2016). Since multidimensional ab initio hydrodynamical simulations of core-collapse supernovae are practically impossible, and the adopted physical effects and approximations, in particular for the neutrino transport, have been rather varied among the models, a consensus on the explosion mechanism has not been reached. Several 3D simulations of core-collapse supernovae have revealed that the strong sloshing motion introduced by the low unstable mode of SASI is not evident, at least at later phases of the shock revival (e.g., Nordhaus et al. 2010b; Dolence et al. 2013; Hanke et al. 2013). Not all but some recent 3D hydrodynamical models have shown an asymmetric dipolarlike morphology (asymmetric two-lobe structure) depending on the progenitor models (Müller et al. 2017; Burrows et al. 2019; Vartanyan et al. 2019; see, e.g., Figure 1 in Vartanyan et al. 2019). In this paper, in the fiducial model (b18.3-high), the parameter set of $\left(\alpha \equiv v_{\text {up }} / v_{\text {down }}, \beta \equiv v_{\text {pol }} / v_{\text {eq }}\right)=(1.5,16)$ is adopted. As seen in Figure 26 (bottom right panel and the case of $\beta=16$ ) in Appendix $\mathrm{A}$, the distribution of initial radial velocities is rather concentrated in the polar direction, which invokes a bipolar but more narrowly collimated (jetlike) explosion than those seen in the models mentioned above (Müller et al. 2017; Burrows et al. 2019; Vartanyan et al. 2019). Such jetlike explosions could be realized by magnetorotationally induced core-collapse supernova explosions (e.g., Takiwaki et al. 2009; Sawai et al. 2013; Mösta et al. 2014). In MHD simulations, both a strong magnetic field and rapid rotation before the core collapse are generally necessary for a successful magnetorotationally induced explosion; however, it has not been revealed yet from which evolutionary paths both conditions are realized simultaneously in the progenitor star just before the core collapse (see, e.g., Heger et al. 2005). To assess whether such magnetorotationally induced explosions can be realized or not, the understanding of the role of the magnetorotational instability (Balbus \& Hawley 1998) is important. Additionally, depending on the circumstances, the combination of magnetorotational and neutrino heating effects could also trigger a jetlike explosion (Sawai \& Yamada 2016).

It is worth noting that even if the explosion is initially narrowly collimated, the morphology is soon modified to be wider at an early phase $(\sim 1 \mathrm{~s})$, as seen in Figure 19 (top left panel), depending on the structure of the $\mathrm{C}+\mathrm{O}$ core of the progenitor star, and the morphology is continuously changed due to the deceleration/acceleration of the blast wave and the growth of instabilities (see Figures 19 and 20). This situation could be much different from that for Type Ia supernovae, where the vestige of the explosion morphology can be survived at even an early phase of the supernova remnant $(\sim 100 \mathrm{yr}$; Ferrand et al. 2019). As seen in Figures 21 and 22, the distribution of ${ }^{56} \mathrm{Ni}$ is time-dependent during the shock propagation in the progenitor star, but the bipolar structure globally survives even after the shock breakout. In the models, e.g., b18.3-high and n16.3-high, the axisymmetric bipolar structures are identified for each element, and heavier elements are more concentrated along the bipolar axis, as seen in Figure 23. Therefore, if such clear axisymmetric structures are identified from the future observations in the spatial distributions of emission lines of elements, in particular ones from iron or direct $\gamma$-ray lines from the decay of ${ }^{44} \mathrm{Ti}$, it would be a clue to deduce the explosion mechanism. Recent observations of 3D distributions of $\mathrm{CO}$ and $\mathrm{SiO}$ molecules in the inner ejecta of SN 1987A (Abellán et al. 2017) are an eligible target for the test. Actually, we have compared the results (approximate $\mathrm{CO}$ and 
$\mathrm{SiO}$ distributions) of 3D MHD simulations of further evolution of models b18.3-high and n16.3-high with the observed CO and $\mathrm{SiO}$ distributions (Orlando et al. 2019b). Additionally, in order to estimate the $\mathrm{CO}$ and $\mathrm{SiO}$ distributions more accurately, we plan to calculate (Ono et al. 2019, in preparation) the molecule formation in the ejecta with a molecule formation network using a post-processing method based on the 3D MHD simulation results above.

\subsection{NS Kick Velocity}

The compact object of SN 1987A has not been detected yet, but it could be a thermally emitting NS obscured by dust (Orlando et al. 2015; Alp et al. 2018). If the explosion of SN 1987A was an asymmetric one, as demonstrated in the models, e.g., b18.3-high, the compact object (probably an NS) could have been kicked in the opposite direction to the motion of the bulk of the supernova ejecta. Actually, NS kicks are expected from 2D and 3D hydrodynamical simulations of neutrinodriven explosions aided by SASI and/or convection thanks to their aspherical nature (Scheck et al. 2004, 2006; Nordhaus et al. 2010a, 2012; Wongwathanarat et al. 2010, 2013). In the context of the neutrino-driven explosion, first, an NS is kicked in the opposite direction to the strongest explosion, and later, the motion is mediated by the interaction between the gravitationally combined, denser, slowly moving clumps left behind the shock and the compact object (gravitational tugboat mechanism; Wongwathanarat et al. 2013). An asymmetric neutrino emission has been proposed as another mechanism of NS kicks (Woosley 1987; Bisnovatyi-Kogan 1993; Socrates et al. 2005). From the X-ray observations of six core-collapse supernova remnants, Cas A, G292.0+1.8, Puppis A, Kes 73, RCW 103, and N49, it has been revealed that the direction of the NS kick relative to the explosion center is opposite to the center of mass of gaseous intermediate elements in the ejecta (Katsuda et al. 2018), which supports a hydrodynamical origin of NS kicks such as the gravitational tugboat mechanism. Recent analysis of the observations of Cas A has indicated that heavier elements are more oppositely distributed than lighter ones (Holland-Ashford et al. 2019). From the observed proper motions of young pulsars, the 3D NS kick velocities of young pulsars have been typically deduced as $300-500 \mathrm{~km} \mathrm{~s}^{-1}$ (Arzoumanian et al. 2002; Brisken et al. 2003; Hobbs et al. 2005; Faucher-Giguère \& Kaspi 2006), but some pulsars have a velocity over $1000 \mathrm{~km} \mathrm{~s}^{-1}$ (e.g., Chatterjee et al. 2005). Motivated by the situation above, we estimate the NS kick velocity by assuming simply momentum conservation (the initial total momentum is zero), as in Wongwathanarat et al. (2013). The NS kick velocity, $\boldsymbol{v}_{\mathrm{NS}}$, is estimated as follows:

$$
\boldsymbol{v}_{\mathrm{NS}}=-\boldsymbol{P}_{\mathrm{gas}} / M_{\mathrm{NS}}=-\int_{V} \rho \boldsymbol{v} d x d y d z / M_{\mathrm{NS}},
$$

where $\boldsymbol{P}_{\text {gas }}$ is the total momentum inside the computational domain, $V$, except for the innermost regions corresponding to the compact object (NS); $M_{\mathrm{NS}}$ is the mass inside the regions of the NS; and $v$ is the fluid velocity. In the ninth column of Table 3 , the absolute values of the estimated NS kick velocity at the end of the simulation, $v_{\mathrm{NS}}$, are listed. The values of $v_{\mathrm{NS}}$ dominantly depend on the parameter $\alpha \equiv v_{\text {up }} / v_{\text {down }}$. The values of $v_{\mathrm{NS}}$ for the models with $\alpha=2.0$, b18.3-mo13, n16.3-mo13, s18.3-mo13, s19.8-mo13, and b18.3-alpha2, are $\sim 420-580 \mathrm{~km} \mathrm{~s}^{-1}$. The value of $v_{\mathrm{NS}}$ for the model with $\alpha=1.0$, b18.3-alpha1, is $\mathcal{O}(1) \mathrm{km} \mathrm{s}^{-1}$. The values of $v_{\mathrm{NS}}$ for the models with $\alpha=1.5$ are $\sim 250-400 \mathrm{~km} \mathrm{~s}^{-1}$, except for model b18.3-ein1.5, for which $E_{\text {in }}=1.5 \times 10^{51} \mathrm{erg}$ and $v_{\mathrm{NS}}$ $\sim 150 \mathrm{~km} \mathrm{~s}^{-1}$. Therefore, overall, the larger the $\alpha$ value, the larger the NS kick velocity. The NS kick velocities also depend on the parameter $\beta \equiv v_{\mathrm{pol}} / v_{\mathrm{eq}}$. For example, as seen in models s18.0-beta2, s18.0-beta4, s18.0-beta8, and s18.0-fid, the larger the $\beta$ value, the smaller the value of $v_{\mathrm{NS}}$, which reflects the fact that if the total kinetic energy is fixed (here uniform density is considered), the wider the bipolar explosion, the larger the net momentum. ${ }^{12}$ Overall, the obtained values of $v_{\mathrm{NS}}$ are roughly within the range of the observed NS kick velocities. The vector values of the NS kick velocities for models b18.3-high and n16.3-high are $\boldsymbol{v}_{\mathrm{NS}}=\left(v_{x}, v_{y}, v_{z}\right)=(-7.23,1.28,-2.85 \times$ $\left.10^{2}\right)$ and $\left(0.103,-0.504,-3.03 \times 10^{2}\right) \mathrm{km} \mathrm{s}^{-1}$, respectively. Then, the values of $v_{x}$ and $v_{y}$ are $\mathcal{O}(1) \mathrm{km} \mathrm{s}^{-1}$, and the NS kick velocities are directed almost opposite to the $z$-axis (the strongest explosion direction). The absolute values for models b18.3-high and n16.3-high are $2.85 \times 10^{2}$ and $3.03 \times$ $10^{2} \mathrm{~km} \mathrm{~s}^{-1}$, respectively, which are consistent with the observed values. As seen in Figure 11 in Katsuda et al. (2018), the relative positions of the compact objects are not perfectly opposed to the positions of the center of mass of the ejecta. Even if the overall features of the observed NS kick velocities can be explained by the hydrodynamical effects demonstrated in this paper, such deviations have not been well explained yet. Rotation, which is not included in this paper, may play an important role. As mentioned in Section 4.3, from the comparison of the line-of-sight velocities of ${ }^{56} \mathrm{Ni}$ with the observed [Fe II] line profiles, the parameter set of $(\theta, \chi)=$ $\left(130^{\circ}, 10^{\circ}\right)$ (see Figure 15$)$, which determines the direction of the bipolar explosion axis (the strongest explosion direction) to observers on Earth, is preferred for the fiducial (best) model, b18.3-high. As seen in Figure 15, if the explosion of the redshifted side (the strongest explosion side) is directed to the south side (negative $x$ direction) to us, as in the case of $(\theta$, $\chi)=\left(130^{\circ}, 10^{\circ}\right)$, the NS kick velocity is directed to the north side. Then, if the best model, b18.3-high with $(\theta, \chi)=$ $\left(130^{\circ}, 10^{\circ}\right)$, is correct, we predict that the compact object of SN 1987A will be found in the northern part of the inner ejecta. Recent observations of dust emission from the inner ejecta of SN 1987A by ALMA have suggested that a dust peak found to the northeast of the center of the remnant could be an indirect detection of the compact object (Cigan et al. 2019), which is very roughly consistent with our prediction.

\subsection{Issues in Stellar Evolution Models and Impacts of Possible Large-density Perturbations in the Progenitor Star}

As presented in Section 4.3, even in the fiducial (best) model, b18.3-high, observed fluxes of [Fe II] lines (points with normalized values of $\gtrsim 0.1$ ) around the high-velocity tails (the absolute Doppler velocity higher than $3000 \mathrm{~km} \mathrm{~s}^{-1}$ ) cannot be reproduced well (see the left panel in Figure 25). Another

\footnotetext{
12 Consider a $1 \mathrm{~cm}^{3}$ cube with a kinetic energy of $\frac{1}{2} \rho v^{2}$. If the kinetic energy is divided into two $1 \mathrm{~cm}^{3}$ cubes, the total kinetic energy is $\frac{1}{2} \rho v^{2}=$ $\frac{1}{2} \rho\left(\frac{v}{\sqrt{2}}\right)^{2}+\frac{1}{2} \rho\left(\frac{v}{\sqrt{2}}\right)^{2}$, whereas the net momentum before dividing is $\rho v$, but the momentum after dividing is $\sqrt{2} \rho v>\rho v$.
} 
possible ingredient that is not included in this paper is large perturbations in density of the pre-supernova models, which were previously investigated in Paper II. Here we discuss the current status of the stellar evolution models and the impact of such large-density perturbations.

Pre-supernova models obtained from stellar evolution calculations are basically spherically symmetric, where 1D (spherical) hydrostatic equations with a mixing-length theory (MLT; Böhm-Vitense 1958) for convection are solved (see, e.g., Kippenhahn \& Weigert 1990). Since convection is inherently involved in the turbulent motion of elements in 3D, the MLT itself has had long-standing issues. In the MLT, the length scale of the mixing of an element (eddy) into the surroundings (mixing length: $l$ ) is "assumed" as $l=\alpha H_{P}$, where $\alpha$ is a free parameter and $H_{P}$ is the local pressure scale height. Related uncertainties on the treatments of so-called semiconvection and overshooting have also been problematic. Semiconvection is a slow mixing process in the dynamically stable region due to the existence of a nonzero gradient of the mean molecular weight but vibrationally unstable (the so-called Ledoux criterion is fulfilled, but the Schwarzschild criterion is not). The treatments of semiconvection and the observational constraints have been investigated for a few decades (e.g., Langer et al. 1985; Spruit 1992; Silva Aguirre et al. 2011; Li 2012; Spruit 2013; Zaussinger \& Spruit 2013). Overshooting is the penetration of elements over a convective zone into a dynamically stable region, which may be the most uncertain process in the context of the MLT, and it has been intensively studied in several aspects (e.g., Rogers et al. 2006; Claret 2007; Deng \& Xiong 2008; Montalbán et al. 2013; Zhang 2013; Viallet et al. 2015). For both the semiconvection and overshooting, nonlocality is essential, and self-consistent nonlocal convection theories beyond the local MLT have been proposed (Xiong 1977; Grossman et al. 1993; Xiong et al. 1997; Canuto \& Dubovikov 1998; Deng et al. 2006; Li \& Yang 2007; Zhang 2016), which has partly been motivated by the implications of the multidimensional hydrodynamical simulations mentioned below. In general, the timescale of the stellar evolution is determined by the nuclear burning, which is much longer than the dynamical timescale of the turbulent motion of fluids in a convective layer and the crossing time of sound waves. Then, it is impossible to cover the whole evolution of a star by multidimensional hydrodynamical simulations (for compressible fluids) in which the time step is limited by the maximum fluid velocity or the maximum sound speed inside the computational domain from the CourantFriedrichs-Lewy condition. Nevertheless, there have been attempts at such multidimensional hydrodynamical simulations of the evolution of massive stars that cover one or a few burning shells (for up to a few convection turnover times in the case of 3D simulations; Bazan \& Arnett 1994; Bazán \& Arnett 1998; Meakin \& Arnett 2006, 2007a, 2007b; Arnett et al. 2009; Arnett \& Meakin 2011a; Viallet et al. 2013; Couch et al. 2015; Chatzopoulos et al. 2016; Müller et al. 2016; Cristini et al. 2017; Mocák et al. 2018; Yoshida et al. 2019). As seen in, e.g., Figures 3 and 4 in Müller et al. (2016), the distributions of ${ }^{28} \mathrm{Si}$ and radial velocities fluctuate a lot with large-scale anisotropies. From the investigations above, for example, Meakin \& Arnett (2007b) argued for turbulent convection, where a turbulent layer adjacent to a stably stratified layer diffuses into the stable layer over time (turbulent entrainment), which is generally ignored in the stellar evolution models based on local MLTs (the authors also suggested that overshooting is best described as an elastic response by the convective boundary). Arnett \& Meakin (2011b) pointed out the " $\tau$-mechanism" as a new source of luminosity fluctuations associated with turbulent convective cells based on 3D hydrodynamical simulations of shell oxygen burning, which exhibits recurrent fluctuations in turbulent kinetic energy. Recently, a new method to replace the MLT in 1D stellar evolutionary computations based on 3D hydrodynamic simulations ("321D" approach) has been presented (Arnett et al. 2015). Keeping in mind the impact of the density fluctuations in the progenitors on the matter mixing, it is interesting to see how a large amplitude of fluctuations could be introduced. Overall, among the multidimensional hydrodynamical simulations, density fluctuations up to $\sim 10 \%$ could be introduced around the edges of the convective zone of the oxygen-burning shell in $\sim 20 M_{\odot}$ stars (Bazán \& Arnett 1998; Meakin \& Arnett 2006, 2007a) and in the envelope of a $5 M_{\odot}$ red giant (Viallet et al. 2013). For lower-mass stars, high-resolution 3D global hydrodynamical simulations of the solar convection (Hotta et al. 2014, 2015) and He shell flash in a post-AGB star (Herwig et al. 2014) have been performed, but the amplitudes of the density fluctuations introduced seem to be small. Smith \& Arnett (2014) discussed the discordance between the predictions from stellar evolution models and the last stages of massive stars, some of which (progenitors of SNe IIn) exhibit eruptive mass ejection a decade before the core collapse. The authors suggested that the major reason for the discordance may lie in the treatments of turbulent convection; i.e., stellar evolution models with MLTs generally ignore (i) finite amplitude fluctuations in velocity and temperature and (ii) their nonlinear interaction with nuclear burning. Such mass ejection invokes more violent eruptions from luminous blue variables (LBVs) such as $\eta$ Carinae. Actually, the candidate of an LBV, HD 168625, is a nearby twin of Sk $-69^{\circ} 202$, which has a similar triple ring structure. From the similarity with HD 168625, Smith (2007) proposed a scenario that Sk $-69^{\circ} 202$ was an LBV evolved as a single star, although the single-star evolution scenario contradicts the binary merger model b18.3, proposed as the pre-supernova model for the fiducial model (b18.3-high) in this paper. It was theoretically demonstrated that some binary mergers are capable of producing LBVs (Justham et al. 2014). Actually, it has been proposed that $\eta$ Carinae and an LVB candidate, R4, currently in a binary system, were derived from a binary merger of two stars originally in a triple star system (for the former and the latter, see Pasquali et al. 2000 and Portegies Zwart \& van den Heuvel 2016, respectively). Whatever the evolution scenario is, violent dynamical eruptions from the envelope of an LBV or mass ejection from a rapidly rotating BSG would cause largescale fluctuations in the envelope. Herschel observations of the closest RSG, Betelgeuse, have revealed that the observed clumpy structure in the inner part of the circumstellar medium could stem from giant convection cells of the outer atmosphere (Decin et al. 2012). The observed close molecular layer and the intensity map computed based on $3 \mathrm{D}$ radiative hydrodynamic simulations of RSGs have also invoked large-scale fluctuations in the envelope of Betelgeuse (Chiavassa et al. 2010; Montargès et al. 2014; see Figure 10 in Montargès et al. 2014). Hitherto, despite intensive attempts at the multidimensional hydrodynamical simulations mentioned above, the theoretical understanding of the stellar envelopes of massive 
stars, in particular at the last stage before the core collapse, is far from conclusive, and it has not been unveiled how large fluctuations can be introduced in the envelopes of presupernova stars. In addition, recent 3D hydrodynamical simulations of core-collapse supernova explosions have revealed that pre-collapse asphericities around $\mathrm{Si} / \mathrm{O}$ layers due to turbulence could alter the post-bounce evolution and enhance the explodability of core-collapse supernovae in the context of the neutrino-driven mechanism (Couch \& Ott 2013, 2015; Müller et al. 2017).

Motivated by the theoretical and observational situations mentioned above, in Paper II, we investigated the influence of large-density perturbations of the amplitude of up to $50 \%$ in the density of the pre-supernova model (same as model n16.3 in this paper) based on 2D hydrodynamical simulations focusing on the matter mixing. Among the investigated models, if there are nonradial perturbations $(50 \%)$ with radially coherent structures (see the top left panel in Figure 2 in Paper II), high-velocity clumps of ${ }^{56} \mathrm{Ni}\left(\lesssim 4000 \mathrm{~km} \mathrm{~s}^{-1}\right)$ can be obtained at the tails of the highest Doppler velocity (see, e.g., Figure 15 in Paper II), even if the explosion is only mildly aspherical (approximately the same as seen in the top left panel in Figure 26 (the case of $\beta=4$ ) in this paper). The high-velocity clumps of ${ }^{56} \mathrm{Ni}$ obtained correspond to the tips of the giant RT fingers. Therefore, by introducing such large-density perturbations in model b18.3-high, a better fit to the observed [Fe II] line profiles may be obtained, although such an investigation is beyond the scope of this paper.

\section{Summary}

In this paper, we perform 3D hydrodynamic simulations of nonspherical core-collapse supernovae, focusing on the matter mixing in SN 1987A. The impact of the four pre-supernova models and parameterized aspherical explosions on the matter mixing are investigated. For the aspherical explosions, we explore asymmetric bipolar explosions characterized by the parameters $\alpha \equiv v_{\text {up }} / v_{\text {down }}$ and $\beta \equiv v_{\text {pol }} / v_{\text {eq }}$. As one of the progenitor models, the BSG pre-supernova model for $\mathrm{Sk}-69^{\circ}$ 202 , based on the slow-merger scenario (b18.3), is adopted, in addition to existing single-star models, one BSG model (n16.3), and the other two RSG models (s18.0 and s19.8). From the simulations results, the radial velocity distribution of elements, in particular ${ }^{56} \mathrm{Ni}$, and the distribution of the line-of-sight velocity of ${ }^{56} \mathrm{Ni}$ are mainly discussed by comparing with the constraints on the mass of high-velocity ${ }^{56} \mathrm{Ni}$ and observed [Fe II] line profiles for SN 1987A. First, we perform 1D simulations in order to see the pre-supernova model dependence of the matter mixing, where the growth factors of instabilities are presented (Section 4.1). Next, we explore the dependence on the parameters of the aspherical explosion and the presupernova models based on many lower-resolution simulations (Section 4.2). Then, with the fiducial (best) parameter set for the explosion, two high-resolution simulations, one with the binary merger progenitor model b18.3 and the other with the single-star progenitor model $\mathrm{n} 16.3$, are performed (Section 4.3). Finally, some implications of the results - the key properties of the presupernova models for the matter mixing (Section 5.1), explosion asymmetries and possible explosion mechanisms for SN 1987A (Section 5.2), NS kick velocities (Section 5.3), and the impacts of possible large-density perturbations in the pre-supernova models - are presented (Section 5.4). Here the findings and main points in this paper are summarized.
1. From the analysis of growth factors of instabilities based on 1D simulations, instabilities grow around both the $\mathrm{C}$ $+\mathrm{O} / \mathrm{He}$ and $\mathrm{He} / \mathrm{H}$ interfaces for the two $\mathrm{BSG}$ progenitor models (b18.3 and n16.3). On the other hand, instabilities are developed only around the $\mathrm{He} / \mathrm{H}$ interfaces for the two RSG progenitor models (s18.0 and s19.8), which is attributed to the fact that the gradients of the $\rho r^{3}$ profile in the helium layer are overall negative for the RSG models, in contrast to the case of the BSG models.

2. Initial asphericities of explosions affect the early $(\sim 1 \mathrm{~s})$ morphologies of the inner ejecta. As expected, the larger the $\beta$ value, the narrower the bipolar structure. However, compared with the initial radial velocity distributions, the morphologies of the bipolar structure at around $\sim 1 \mathrm{~s}$ become wider and less distinct due to the deceleration during the shock propagation inside the $\mathrm{C}+\mathrm{O}$ core.

3. The early morphologies of the expanding ejecta depend on the structures in the pre-supernova models. At an early phase $(\sim 1 \mathrm{~s})$, depending on the gradients of the $\rho r^{3}$ profiles of the $\mathrm{C}+\mathrm{O}$ and/or the silicon layers, presupernova models with steeper $\rho r^{3}$ gradients result in a wider bipolar structure in the early morphology of the explosion due to stronger decelerations than those for progenitor models with flatter or negative gradients. The BSG progenitor model based on the single-star evolution (n16.3) results in the widest bipolar structure in the ejecta.

4. Later morphologies of the expanding ejecta and the distributions of elements also depend on the structures of the helium and hydrogen layers of the pre-supernova models. The distributions of lower-mass elements, e.g., ${ }^{16} \mathrm{O}$ and ${ }^{4} \mathrm{He}$, depend on whether the reverse shock developed during the shock propagation in the hydrogen layer sweeps up the inner ejecta or not. In the BSG model based on the binary merger evolution (b18.3), the reverse shock last developed sweeps up the inner ejecta completely by the time of the shock breakout. Consequently, the distributions of ${ }^{16} \mathrm{O}$ and ${ }^{4} \mathrm{He}$ are more concentrated around the equatorial plane.

5. Among the investigated explosion models, the models with the pre-supernova model n16.3 fail to simultaneously fulfill the two observational constraints on the mass of the high-velocity ${ }^{56} \mathrm{Ni}$, i.e., (i) $M_{3.0} / M_{\mathrm{ej}}$ $\left({ }^{56} \mathrm{Ni}\right) \geqslant 4 \%$ and (ii) $M_{4.0} \quad\left({ }^{56} \mathrm{Ni}\right) \geqslant 10^{-3} M_{\odot}$ (see Table 3 and Figure 11). On the other hand, some explosion models with the other pre-supernova models succeed in fulfilling the observational constraints for the case of larger $\beta$ values ( 8 or 16).

6. If the explosion models with RSG models and extreme explosion energies are excluded from the point of view of the observational constraints on the progenitor of SN 1987A, Sk $-69^{\circ} 202$, and its explosion, the best model in this paper is model b18.3-high, in which the binary merger progenitor model b18.3 (Urushibata et al. 2018) and the parameter set of $(\alpha, \beta)=(1.5,16)$ are adopted. In the best model, the obtained explosion energy, $E_{\text {exp }}$, is $\sim 2 \times 10^{51}$ erg.

7. The obtained values related to the observational constraints on the mass of the high-velocity ${ }^{56} \mathrm{Ni}, M_{3.0} / M_{\mathrm{ej}}$ $\left({ }^{56} \mathrm{Ni}\right)$ and $M_{4.0}\left({ }^{56} \mathrm{Ni}\right)$, for the best explosion model, b18.3-high, are $10.5 \%$ and $1.1 \times 10^{-3} M_{\odot}$, respectively. The values for the counterpart model n16.3-high, in 
which the single-star progenitor model n16.3 is adopted, are $3.7 \%$ and $2.5 \times 10^{-4} M_{\odot}$, respectively.

8. The distribution of the line-of-sight velocity of ${ }^{56} \mathrm{Ni}$ for model b18.3-high best reproduces the high-velocity tails of the observed [Fe II] line profiles, in particular at the redshifted side with angles of $(\theta, \chi)=\left(130^{\circ}, 10^{\circ}\right.$; see Figure 15 for the definition of the angles and Figure 25 for the distribution). The distribution for the counterpart model n16.3 apparently lacks the tail at the redshifted side.

9. The key to obtaining such high-velocity ${ }^{56} \mathrm{Ni}$ is the penetration of ${ }^{56} \mathrm{Ni}$ through the helium layer to reach the hydrogen envelope before the development of the strong reverse shock during the shock propagation in the helium layer, which is consistent with the findings in Wongwathanarat et al. (2015).

10. To realize the penetration of ${ }^{56} \mathrm{Ni}$ through the helium layer, the structures of the $\mathrm{C}+\mathrm{O}$ and the helium layers are important. At least among the existing BSG progenitor models, including models b18.3 and $\mathrm{n} 16.3$, the helium core mass $M_{\mathrm{He}, \mathrm{c}}$ or the mass ratio of the helium core to the stellar mass $q \equiv M_{\mathrm{He}, \mathrm{c}} / M$ appears to be a useful indicator for the successful penetration of ${ }^{56} \mathrm{Ni}$, as seen in previous studies (Utrobin et al. 2015, 2019; Wongwathanarat et al. 2015). The value of $M_{\mathrm{He}, \mathrm{c}}$ of the pre-supernova model for the best explosion model b18.3high is about $\sim 4 M_{\odot}(q \sim 0.2)$. On the other hand, the value for the other BSG model, $\mathrm{n} 16.3$, is $\sim 6 M_{\odot}$ $(q \sim 0.37)$.

11. It seems difficult to find such a small $M_{\mathrm{He}, \mathrm{c}}$ value among the existing BSG progenitor models based on the singlestar evolution that satisfy both the observed luminosity and the effective temperature (the final position in the H-R diagram) of Sk $-69^{\circ} 202$. On the other hand, the existing BSG models based on the binary merger scenario that satisfy these values naturally have small $M_{\mathrm{He}, \mathrm{c}}$ values of $\lesssim 4 M_{\odot}(q \lesssim 0.2$; Menon \& Heger 2017; Urushibata et al. 2018), which may reflect the nature of merging processes, the penetration of the secondary to the envelope of the primary, and the dredge-up of the primary's core material into the envelope.

12. From the adopted parameter set of the best explosion model, b18.3-high, the explosion of SN 1987A is likely to be an asymmetric bipolar (jetlike) explosion, which may be induced by magnetorotational effects (e.g., Takiwaki et al. 2009; Sawai et al. 2013; Mösta et al. 2014) or the combination of the neutrino heating and the magnetorotational effects (Sawai \& Yamada 2016). In order to deduce the explosion mechanism in a more robust way, observations of spatially resolved line emissions from iron or direct $\gamma$-ray lines from the decay of ${ }^{44} \mathrm{Ti}$ are desirable. Recent observations of the 3D distribution of $\mathrm{CO}$ and $\mathrm{SiO}$ molecules (Abellán et al. 2017) will shed light on the explosion mechanism.

13. From the asymmetric bipolar explosions presented in this paper, NS kicks are expected, as in Wongwathanarat et al. (2013). The absolute value of the estimated NS kick velocity, $v_{\mathrm{NS}}$, for the best model, b18.3, is $\sim 300 \mathrm{~km} \mathrm{~s}^{-1}$. The values for the other models are roughly in the range of $250-580 \mathrm{~km} \mathrm{~s}^{-1}$ (except for models b18.3-alpha1 and b18.3-ein1.5), which is consistent with the NS kick velocities deduced from the proper motions of young pulsars (e.g., Hobbs et al. 2005; Faucher-Giguère \& Kaspi 2006). It is found that the direction of the NS kick is almost opposite to the bipolar (strongest) explosion axis. From the angles suggested by the best model, b18.3-high, $(\theta, \chi)=\left(130^{\circ}, 10^{\circ}\right)$, we predict that the compact object of SN 1987A will be detected in the north part of the inner ejecta, as opposed to the direction of the redshifted side of the explosion, which corresponds to the stronger explosion direction and is directed to the south side (see Figure 15).

14. As investigated in Paper II, possible large-density fluctuations with amplitudes up to 50\% in the presupernova model could aid the inner ejecta in penetrating through the helium layer due to strong RT instabilities. Hitherto, whether such a large amplitude of fluctuations can be introduced in the density of pre-supernova models or not has not been unveiled because of the lack of appropriate theoretical modeling of multidimensional effects such as turbulent convection, in particular for the envelope at the last stage before the core collapse. It is worth investigating such effects (partly) motivated by the recent explorations of the impact of pre-collapse asphericities on the core-collapse supernova explosions (e.g., Couch \& Ott 2015; Müller et al. 2017).

We have made use of the results of models b18.3-high and n16.3-high as initial conditions of MHD simulations of further evolution (up to $\sim 50$ yr for SN 1987A; Orlando et al. 2019b), which is a natural extension of our previous investigations with spherically symmetric explosions (Orlando et al. 2015, 2019a; Miceli et al. 2019). In the paper, we have discussed not only the $\mathrm{X}$-ray emission (the light curve and the images) but also the distributions of $\mathrm{CO}$ and $\mathrm{SiO}$ molecules motivated by the recently observed 3D distributions of the molecules (Abellán et al. 2017). Additionally, we plan to investigate the molecule and/or dust formations in detail based on the 3D models in this paper in the near future (M. Ono et al. 2020, in preparation).

We would like to thank M. Barkov, D. C. Warren, and T. Nozawa for fruitful discussion on this work. The software used in this work was in part developed by the DOE NNSA-ASC OASCR Flash Center at the University of Chicago. The numerical computations were carried out complementarily on the XC40 (YITP, Kyoto University), Cray XC50 (Center for Computational Astrophysics, National Astronomical Observatory of Japan), and HOKUSAI (RIKEN). This research also used the computational resources of the $\mathrm{K}$ computer provided by the RIKEN Center for Computational Science through the HPCI System Research project (Project ID: hp180281) and the PRACE Research Infrastructure resource Marconi based in Italy at CINECA (PRACE Award No. 2016153460). This work is supported by JSPS KAKENHI grant Nos. JP26800141 and JP19H00693. M.O. and S.N. are thankful for the support of the RIKEN Interdisciplinary Theoretical and Mathematical Sciences Program. S.N. also acknowledges the support of the Pioneering Program of RIKEN for Evolution of Matter in the Universe (r-EMU).

\section{Appendix A \\ Explosion Asymmetries: Initial Radial Velocity Distributions}

In the simulation, thermal and kinetic energies are artificially injected around the interface between the iron core and the 

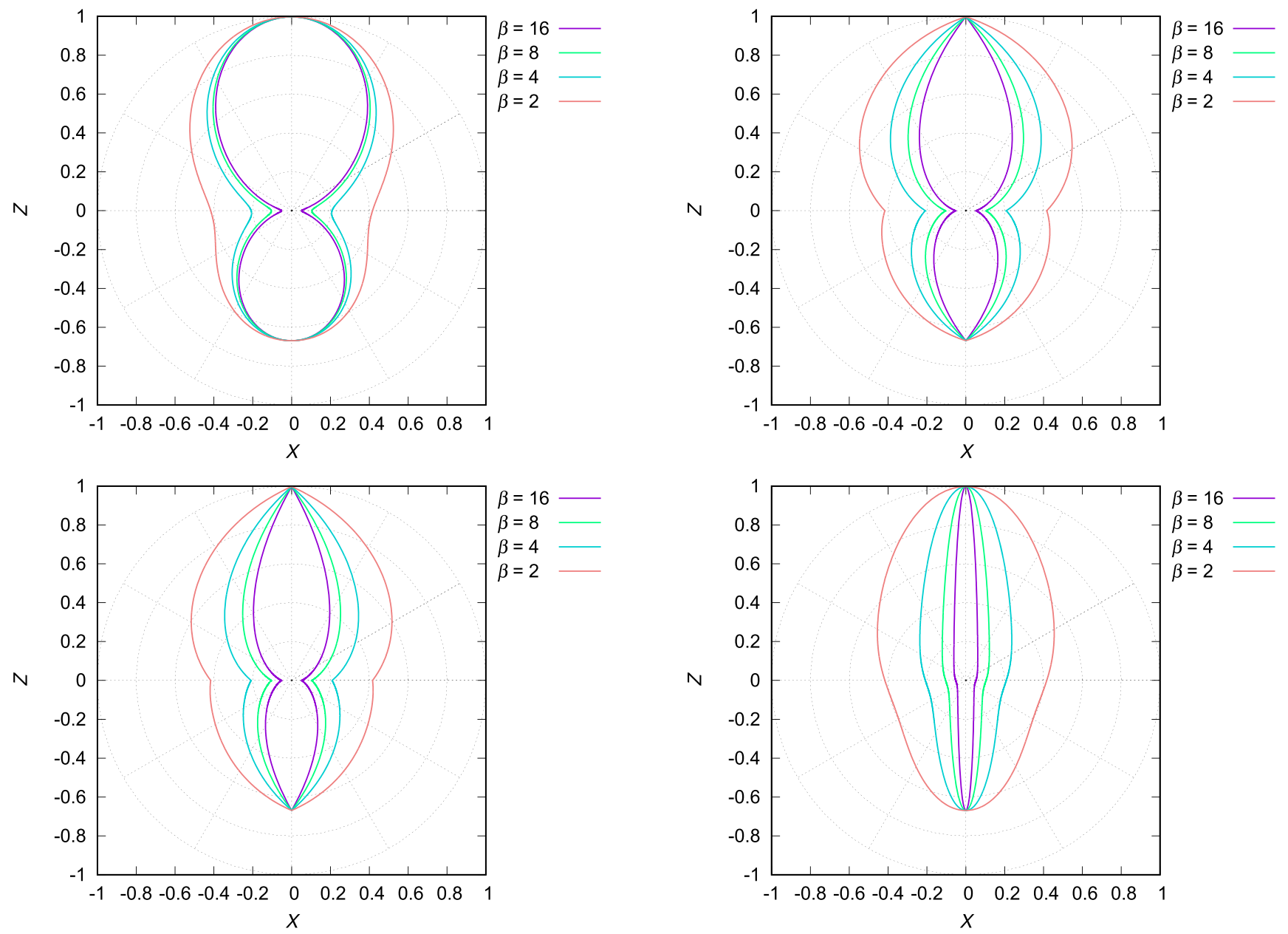

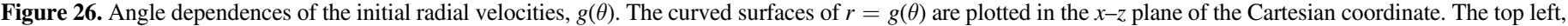

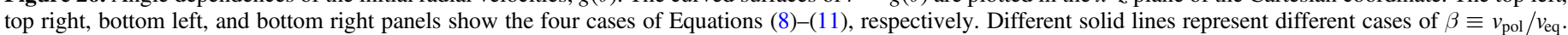
The case of $\alpha \equiv v_{\text {up }} / v_{\text {down }}=1.5$ is shown.

silicon layer of the pre-supernova models. In order to initiate an asymmetric (nonspherical) explosion, initial radial velocities are distributed with arbitrary functions of $\theta$ in the spherical coordinates $(r, \theta, \phi)$. In this paper, we assume the form of the initial radial velocities as $v_{r} \propto r f(\theta)$. Since observations of SN 1987A have shown that the inner ejecta is globally elliptical (Kjær et al. 2010; Larsson et al. 2013, 2016), bipolar-like explosions may be justified. Then, the following four cases for the shape of $f(\theta)(0 \leqslant \theta \leqslant \pi)$ are considered. In the four cases, the concentration of higher initial radial velocities around the polar axis is controlled by a parameter, $\beta \equiv v_{\mathrm{pol}} / v_{\mathrm{eq}}$, where $v_{\mathrm{pol}}$ is the initial radial velocity on the polar axis $(\theta=0)$ and $v_{\mathrm{eq}}$ is one on the equatorial plane $(\theta=\pi / 2)$ at the same radius, $r$.

Case 1: a function with cosine ("cos" in Table 2) as

$$
f(\theta)=\frac{1+\cos (2 \theta)}{1+\xi}
$$

where $\xi$ is a parameter related to $\beta$ with the relation $\beta=(1+\xi) /(1-\xi)$. This form of asymmetry was adopted in Nagataki et al. (1998b) and Paper I.
Case 2: an exponential form ("exponential" in Table 2) as

$$
\begin{gathered}
f(\theta)=\exp (-\vartheta / d), \quad d=\frac{\pi}{2 \ln \beta}, \\
\vartheta= \begin{cases}\theta & (\theta \leqslant \pi / 2) \\
\pi-\theta & (\theta>\pi / 2) .\end{cases}
\end{gathered}
$$

Case 3: a power-law-like form ("power" in Table 2) as

$$
\begin{aligned}
f(\theta) & =\left(2-\frac{2}{\pi} \vartheta\right)^{\gamma}, \quad \gamma=\log _{2} \beta \\
\vartheta & = \begin{cases}\theta & (\theta \leqslant \pi / 2) \\
\pi-\theta & (\theta>\pi / 2) .\end{cases}
\end{aligned}
$$

Case 4: an elliptical form ("elliptical" in Table 2) as

$$
f(\theta)=\left(\beta^{-1} \cos ^{2} \theta+\beta \sin ^{2} \theta\right)^{-1 / 2} .
$$

We also introduce an asymmetry in the initial radial velocities across the equatorial plane $(x-y)$. Such an asymmetry could be introduced if an explosion is driven by neutrino heating aided by the SASI of a low-order unstable mode $(l=1$; e.g., Scheck et al. 2006; Suwa et al. 2010; Hanke et al. 2013; Pan et al. 2016) and could trigger an NS kick inferred from the 
observations of young supernova remnants (Katsuda et al. 2018). The initial radial velocities in the upper hemisphere are manually enhanced by multiplying the factor of $\alpha \equiv v_{\text {up }} / v_{\text {down }}$, and the velocities around the equatorial plane are smoothed so as not to introduce a jump across the plane. We denote the angle dependences after a normalization as $g(\theta)$, where the function is normalized in order for the maximum value to be unity. The angle dependences, $g(\theta)$, are shown in Figure 26. The cases of $\beta=2,4,8$, and 16 with $\alpha=1.5$ are displayed in the $x-z$ plane of the Cartesian coordinate system. As one can see, for example, the distribution of the elliptical case with $\beta=16$ (bottom right panel) invokes a rather jetlike explosion. Then, at this moment, we do not assume a specific explosion mechanism for this initial radial velocity distribution.

\section{Appendix B \\ Method for Introducing the Initial Clumpy Structures}

In our previous study on the matter mixing based on 2D hydrodynamic simulations (Paper I) motivated by neutrinodriven explosions aided by SASI and/or convection, which have clumpy or bubble-like structures inside the supernova shock wave, fluctuations were introduced in the initial radial velocities by multiplying the following factor function of $\theta$ :

$$
1+\sum_{n=1}^{4} \frac{\epsilon}{2^{n-1}} \sin (m n \theta)
$$

where $\epsilon$ is the amplitude of the fluctuations and $m$ is an integer parameter. In the best model for SN 1987A in Paper I (AM2), $m=15$ was adopted. In this paper, the simulations are 3D. Thus, we introduce such fluctuations with another function of $(\theta, \phi)$. We utilize real spherical harmonics for the function:

$$
\begin{aligned}
& Y_{l}^{m}(\theta, \phi) \\
& = \begin{cases}(-1)^{m} \sqrt{2} \sqrt{\frac{2 l+1}{4 \pi} \frac{(l-|m|) !}{(l+|m|) !}} P_{l}^{|m|}(\cos \theta) \sin (|m| \phi) & (m<0) \\
\sqrt{\frac{2 l+1}{4 \pi}} P_{l}^{m}(\cos \theta) & (m=0) \\
(-1)^{m} \sqrt{2} \sqrt{\frac{2 l+1}{4 \pi} \frac{(l-m) !}{(l+m) !}} P_{l}^{m}(\cos \theta) \cos (m \phi) & (m>0) .\end{cases}
\end{aligned}
$$

With the function above, the following factor is multiplied to the initial radial velocities introduced in Appendix A:

$$
1+\epsilon\left[N \sum_{n=1}^{4} \sum_{m=-l}^{l} \frac{A_{l}^{m}(\theta, \phi)}{n}\right]\left(l=n \cdot l_{\text {base }}\right),
$$

where $\epsilon$ is the amplitude of the fluctuations. In order to introduce fluctuations of similar sizes and amplitudes as in the best model (AM2) in Paper I, $l_{\text {base }}=15$ and $\epsilon=30 \%$ are adopted. It is noted that nonradial fluctuations with an amplitude of $\sim 30 \%$ could be introduced as seen in a 2D hydrodynamical simulation of a neutrino-driven core-collapse supernova explosion (see Figure 11 in Gawryszczak et al. 2010). Here $N$ is a normalization factor for the maximum value inside the square bracket in Equation (14) to be unity. The function, $A_{l}^{m}(\theta, \phi)$, is basically the function in Equation (13), but depending on the numbers of $l$ and $m$, some values are arbitrarily set to be zero (some $m$ modes are arbitrarily selected), as follows:

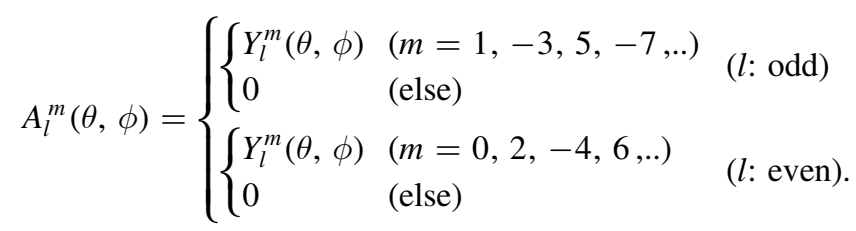

There is no physical basis for the selection of $m$ modes, but it is noted that if we set $A_{l}^{m}(\theta, \phi)$ to be $Y_{l}^{m}(\theta, \phi)$, the distribution of the fluctuations seems to be unrealistic (nearly axisymmetric stripes are recognized). In this paper, we do not intend to discuss the effects of the specific form of the fluctuations, but just the effects of the existence of such initial fluctuations is briefly argued by comparing one of the models with the fluctuations (b18.3-fid) and one without them (b18.3-clp0).

\section{ORCID iDs}

Masaomi Ono (1D https://orcid.org/0000-0002-0603-918X Shigehiro Nagataki (ib https://orcid.org/0000-0002-7025-284X Koh Takahashi (D) https://orcid.org/0000-0002-6705-6303 Salvatore Orlando (iD https://orcid.org/0000-0003-2836-540X Marco Miceli (D) https://orcid.org/0000-0003-0876-8391

\section{References}

Abellán, F. J., Indebetouw, R., Marcaide, J. M., et al. 2017, ApJL, 842, L24 Ahmad, I., Greene, J. P., Moore, E. F., et al. 2006, PhRvC, 74, 065803

Alp, D., Larsson, J., Fransson, C., et al. 2018, ApJ, 864, 174

Alp, D., Larsson, J., Maeda, K., et al. 2019, ApJ, 882, 22

Arnett, D., Fryxell, B., \& Mueller, E. 1989a, ApJL, 341, L63

Arnett, D., Meakin, C., \& Young, P. A. 2009, ApJ, 690, 1715

Arnett, W. D., Bahcall, J. N., Kirshner, R. P., \& Woosley, S. E. 1989b, ARA\&A, 27, 629

Arnett, W. D., \& Meakin, C. 2011a, ApJ, 733, 78

Arnett, W. D., \& Meakin, C. 2011b, ApJ, 741, 33

Arnett, W. D., Meakin, C., Viallet, M., et al. 2015, ApJ, 809, 30

Arzoumanian, Z., Chernoff, D. F., \& Cordes, J. M. 2002, ApJ, 568, 289

Balbus, S. A., \& Hawley, J. F. 1998, RvMP, 70, 1

Bandiera, R. 1984, A\&A, 139, 368

Bazan, G., \& Arnett, D. 1994, ApJL, 433, L41

Bazán, G., \& Arnett, D. 1998, ApJ, 496, 316

Bear, E., \& Soker, N. 2018, MNRAS, 478, 682

Benz, W., \& Thielemann, F.-K. 1990, ApJL, 348, L17

Bisnovatyi-Kogan, G. S. 1993, A\&AT, 3, 287

Blinnikov, S., Lundqvist, P., Bartunov, O., Nomoto, K., \& Iwamoto, K. 2000, ApJ, 532, 1132

Blondin, J. M., Mezzacappa, A., \& DeMarino, C. 2003, ApJ, 584, 971

Boggs, S. E., Harrison, F. A., Miyasaka, H., et al. 2015, Sci, 348, 670

Böhm-Vitense, E. 1958, ZAp, 46, 108

Brisken, W. F., Fruchter, A. S., Goss, W. M., Herrnstein, R. M., \& Thorsett, S. E. 2003, AJ, 126, 3090

Bruenn, S. W., Lentz, E. J., Hix, W. R., et al. 2016, ApJ, 818, 123

Bruenn, S. W., Mezzacappa, A., Hix, W. R., et al. 2013, ApJL, 767, L6

Burrows, A. 2013, RvMP, 85, 245

Burrows, A., Dessart, L., Livne, E., Ott, C. D., \& Murphy, J. 2007, ApJ, 664,416

Burrows, A., Hayes, J., \& Fryxell, B. A. 1995a, ApJ, 450, 830

Burrows, A., Radice, D., \& Vartanyan, D. 2019, MNRAS, 485, 3153

Burrows, C. J., Krist, J., Hester, J. J., et al. 1995b, ApJ, 452, 680

Canuto, V. M., \& Dubovikov, M. 1998, ApJ, 493, 834

Chatterjee, S., Vlemmings, W. H. T., Brisken, W. F., et al. 2005, ApJL, 630, L61

Chatzopoulos, E., Couch, S. M., Arnett, W. D., \& Timmes, F. X. 2016, ApJ, 822,61

Chevalier, R. A. 1976, ApJ, 207, 872

Chiavassa, A., Haubois, X., Young, J. S., et al. 2010, A\&A, 515, A12 
Chita, S. M., Langer, N., van Marle, A. J., García-Segura, G., \& Heger, A. 2008, A\&A, 488, L37

Cigan, P., Matsuura, M., Gomez, H. L., et al. 2019, ApJ, 886, 51

Claret, A. 2007, A\&A, 475, 1019

Colgan, S. W. J., Haas, M. R., Erickson, E. F., Lord, S. D., \& Hollenbach, D. J. 1994, ApJ, 427, 874

Couch, S. M., Chatzopoulos, E., Arnett, W. D., \& Timmes, F. X. 2015, ApJL, 808, L21

Couch, S. M., \& O’Connor, E. P. 2014, ApJ, 785, 123

Couch, S. M., \& Ott, C. D. 2013, ApJL, 778, L7

Couch, S. M., \& Ott, C. D. 2015, ApJ, 799, 5

Cristini, A., Meakin, C., Hirschi, R., et al. 2017, MNRAS, 471, 279

Crotts, A. P., \& Heathcote, S. R. 1991, Natur, 350, 683

Crotts, A. P. S., \& Heathcote, S. R. 2000, ApJ, 528, 426

Decin, L., Cox, N. L. J., Royer, P., et al. 2012, A\&A, 548, A113

Deng, L., \& Xiong, D. R. 2008, MNRAS, 386, 1979

Deng, L., Xiong, D. R., \& Chan, K. L. 2006, ApJ, 643, 426

Dolence, J. C., Burrows, A., Murphy, J. W., \& Nordhaus, J. 2013, ApJ, 765,110

Dotani, T., Hayashida, K., Inoue, H., et al. 1987, Natur, 330, 230

Ebisuzaki, T., Shigeyama, T., \& Nomoto, K. 1989, ApJL, 344, L65

Ellinger, C. I., Young, P. A., Fryer, C. L., \& Rockefeller, G. 2012, ApJ, 755,160

Esposito, P., Rea, N., Lazzati, D., et al. 2018, ApJ, 857, 58

Faucher-Giguère, C.-A., \& Kaspi, V. M. 2006, ApJ, 643, 332

Ferrand, G., Warren, D. C., Ono, M., et al. 2019, ApJ, 877, 136

France, K., McCray, R., Penton, S. V., et al. 2011, ApJ, 743, 186

Fransson, C., Cassatella, A., Gilmozzi, R., et al. 1989, ApJ, 336, 429

Fryxell, B., Mueller, E., \& Arnett, D. 1991, ApJ, 367, 619

Fryxell, B., Olson, K., Ricker, P., et al. 2000, ApJS, 131, 273

Fujimoto, S.-i., Kotake, K., Hashimoto, M.-a., Ono, M., \& Ohnishi, N. 2011, ApJ, 738, 61

Gawryszczak, A., Guzman, J., Plewa, T., \& Kifonidis, K. 2010, A\&A, 521, A38

Grebenev, S. A., Lutovinov, A. A., Tsygankov, S. S., \& Winkler, C. 2012, Natur, 490, 373

Grossman, S. A., Narayan, R., \& Arnett, D. 1993, ApJ, 407, 284

Haas, M. R., Colgan, S. W. J., Erickson, E. F., et al. 1990, ApJ, 360, 257

Hachisu, I., Matsuda, T., Nomoto, K., \& Shigeyama, T. 1990, ApJL, 358, L57

Hachisu, I., Matsuda, T., Nomoto, K., \& Shigeyama, T. 1992, ApJ, 390, 230

Hammer, N. J., Janka, H.-T., \& Müller, E. 2010, ApJ, 714, 1371

Handy, T., Plewa, T., \& Odrzywołek, A. 2014, ApJ, 783, 125

Hanke, F., Müller, B., Wongwathanarat, A., Marek, A., \& Janka, H.-T. 2013, ApJ, 770, 66

Hanuschik, R. W., Thimm, G., \& Dachs, J. 1988, MNRAS, 234, 41P

Heger, A., \& Langer, N. 1998, A\&A, 334, 210

Heger, A., Woosley, S. E., \& Spruit, H. C. 2005, ApJ, 626, 350

Herant, M., \& Benz, W. 1991, ApJL, 370, L81

Herant, M., \& Benz, W. 1992, ApJ, 387, 294

Herant, M., Benz, W., Hix, W. R., Fryer, C. L., \& Colgate, S. A. 1994, ApJ, 435,339

Herwig, F., Woodward, P. R., Lin, P.-H., Knox, M., \& Fryer, C. 2014, ApJL, 792, L3

Hillebrandt, W., \& Meyer, F. 1989, A\&A, 219, L3

Hobbs, G., Lorimer, D. R., Lyne, A. G., \& Kramer, M. 2005, MNRAS, 360, 974

Holland-Ashford, T., Lopez, L. A., \& Auchettl, K. 2019, arXiv:1904.06357

Hotta, H., Rempel, M., \& Yokoyama, T. 2014, ApJ, 786, 24

Hotta, H., Rempel, M., \& Yokoyama, T. 2015, ApJ, 798, 51

Humphreys, R. M., \& McElroy, D. B. 1984, ApJ, 284, 565

Hungerford, A. L., Fryer, C. L., \& Rockefeller, G. 2005, ApJ, 635, 487

Hungerford, A. L., Fryer, C. L., \& Warren, M. S. 2003, ApJ, 594, 390

Ivanova, N., Podsiadlowski, P., \& Spruit, H. 2002, MNRAS, 334, 819

Janka, H.-T. 2012, ARNPS, 62, 407

Janka, H.-T., Hanke, F., Hüdepohl, L., et al. 2012, PTEP, 2012, 01 A309

Jerkstrand, A., Timmes, F. X., Magkotsios, G., et al. 2015, ApJ, 807, 110

Joggerst, C. C., Almgren, A., Bell, J., et al. 2010a, ApJ, 709, 11

Joggerst, C. C., Almgren, A., \& Woosley, S. E. 2010b, ApJ, 723, 353

Joggerst, C. C., Woosley, S. E., \& Heger, A. 2009, ApJ, 693, 1780

Justham, S., Podsiadlowski, P., \& Vink, J. S. 2014, ApJ, 796, 121

Katsuda, S., Morii, M., Janka, H.-T., et al. 2018, ApJ, 856, 18

Kifonidis, K., Plewa, T., Janka, H.-T., \& Müller, E. 2000, ApJL, 531, L123

Kifonidis, K., Plewa, T., Janka, H.-T., \& Müller, E. 2003, A\&A, 408, 621

Kifonidis, K., Plewa, T., Scheck, L., Janka, H.-T., \& Müller, E. 2006, A\&A, 453,661
Kippenhahn, R., \& Weigert, A. 1990, Stellar Structure and Evolution (Berlin: Springer), 192

Kjær, K., Leibundgut, B., Fransson, C., Jerkstrand, A., \& Spyromilio, J. 2010, A\&A, 517, A51

Kotake, K., Sawai, H., Yamada, S., \& Sato, K. 2004, ApJ, 608, 391

Kotake, K., Sumiyoshi, K., Yamada, S., et al. 2012a, PTEP, 2012, 01A301

Kotake, K., Takiwaki, T., Suwa, Y., et al. 2012b, AdAst, 2012, 39

Kozma, C., \& Fransson, C. 1998, ApJ, 497, 431

Kuroda, T., Kotake, K., \& Takiwaki, T. 2012, ApJ, 755, 11

Langer, N., El Eid, M. F., \& Fricke, K. J. 1985, A\&A, 145, 179

Larsson, J., Fransson, C., Alp, D., et al. 2019, arXiv:1910.09582

Larsson, J., Fransson, C., Kjaer, K., et al. 2013, ApJ, 768, 89

Larsson, J., Fransson, C., Spyromilio, J., et al. 2016, ApJ, 833, 147

Li, Y. 2012, ApJ, 756, 37

Li, Y., \& Yang, J. Y. 2007, MNRAS, 375, 388

Lundqvist, P., \& Fransson, C. 1996, ApJ, 464, 924

MacNeice, P., Olson, K. M., Mobarry, C., de Fainchtein, R., \& Packer, C. 2000, CoPhC, 126, 330

Mao, J., Ono, M., Nagataki, S., et al. 2015, ApJ, 808, 164

Marek, A., \& Janka, H.-T. 2009, ApJ, 694, 664

Mattila, S., Lundqvist, P., Gröningsson, P., et al. 2010, ApJ, 717, 1140

Matz, S. M., Share, G. H., Leising, M. D., et al. 1988, Natur, 331, 416

McCray, R. 1993, ARA\&A, 31, 175

McCray, R., \& Fransson, C. 2016, ARA\&A, 54, 19

Meakin, C. A., \& Arnett, D. 2006, ApJL, 637, L53

Meakin, C. A., \& Arnett, D. 2007a, ApJ, 665, 690

Meakin, C. A., \& Arnett, D. 2007b, ApJ, 667, 448

Menon, A., \& Heger, A. 2017, MNRAS, 469, 4649

Menon, A., Utrobin, V., \& Heger, A. 2019, MNRAS, 482, 438

Miceli, M., Orlando, S., Burrows, D. N., et al. 2019, NatAs, 3, 236

Mocák, M., Meakin, C., Campbell, S. W., \& Arnett, W. D. 2018, MNRAS, 481, 2918

Montalbán, J., Miglio, A., Noels, A., et al. 2013, ApJ, 766, 118

Montargès, M., Kervella, P., Perrin, G., et al. 2014, A\&A, 572, A17

Morris, T., \& Podsiadlowski, P. 2007, Sci, 315, 1103

Morris, T., \& Podsiadlowski, P. 2009, MNRAS, 399, 515

Mösta, P., Richers, S., Ott, C. D., et al. 2014, ApJL, 785, L29

Mueller, E., Fryxell, B., \& Arnett, D. 1991, A\&A, 251, 505

Müller, B. 2016, PASA, 33, e048

Müller, B., Janka, H.-T., \& Heger, A. 2012a, ApJ, 761, 72

Müller, B., Janka, H.-T., \& Marek, A. 2012b, ApJ, 756, 84

Müller, B., Melson, T., Heger, A., \& Janka, H.-T. 2017, MNRAS, 472, 491

Müller, B., Viallet, M., Heger, A., \& Janka, H.-T. 2016, ApJ, 833, 124

Müller, E., Janka, H.-T., \& Wongwathanarat, A. 2012c, A\&A, 537, A63

Nadyozhin, D. K. 1994, ApJS, 92, 527

Nagakura, H., Iwakami, W., Furusawa, S., et al. 2017, ApJS, 229, 42

Nagataki, S. 2000, ApJS, 127, 141

Nagataki, S., Hashimoto, M.-a., Sato, K., \& Yamada, S. 1997, ApJ, 486, 1026

Nagataki, S., Hashimoto, M.-a., Sato, K., Yamada, S., \& Mochizuki, Y. S. 1998a, ApJL, 492, L45

Nagataki, S., Shimizu, T. M., \& Sato, K. 1998b, ApJ, 495, 413

Nakamura, K., Takiwaki, T., Kotake, K., \& Nishimura, N. 2014, ApJ, 782, 91

Nomoto, K., \& Hashimoto, M. 1988, PhR, 163, 13

Nordhaus, J., Brandt, T. D., Burrows, A., \& Almgren, A. 2012, MNRAS, 423, 1805

Nordhaus, J., Brandt, T. D., Burrows, A., Livne, E., \& Ott, C. D. 2010a, PhRvD, 82, 103016

Nordhaus, J., Burrows, A., Almgren, A., \& Bell, J. 2010b, ApJ, 720, 694

Ono, M., Nagataki, S., Ito, H., et al. 2013, ApJ, 773, 161

Orlando, S., Miceli, M., Petruk, O., et al. 2019a, A\&A, 622, A73

Orlando, S., Miceli, M., Pumo, M. L., \& Bocchino, F. 2015, ApJ, 810, 168

Orlando, S., Ono, M., Nagataki, S., et al. 2019b, arXiv:1912.03070

Ott, C. D., Abdikamalov, E., Mösta, P., et al. 2013, ApJ, 768, 115

Pan, K.-C., Liebendörfer, M., Hempel, M., \& Thielemann, F.-K. 2016, ApJ, 817,72

Pasquali, A., Nota, A., Langer, N., Schulte-Ladbeck, R. E., \& Clampin, M. 2000, AJ, 119, 1352

Pinto, P. A., \& Woosley, S. E. 1988, ApJ, 329, 820

Podsiadlowski, P. 1992, PASP, 104, 717

Podsiadlowski, P., Joss, P. C., \& Hsu, J. J. L. 1992, ApJ, 391, 246

Podsiadlowski, P., Joss, P. C., \& Rappaport, S. 1990, A\&A, 227, L9

Portegies Zwart, S. F., \& van den Heuvel, E. P. J. 2016, MNRAS, 456, 3401

Potter, T. M., Staveley-Smith, L., Reville, B., et al. 2014, ApJ, 794, 174

Radice, D., Burrows, A., Vartanyan, D., Skinner, M. A., \& Dolence, J. C. 2017, ApJ, 850, 43

Radice, D., Ott, C. D., Abdikamalov, E., et al. 2016, ApJ, 820, 76 
Rauscher, T., Heger, A., Hoffman, R. D., \& Woosley, S. E. 2002, ApJ, 576, 323

Rogers, T. M., Glatzmaier, G. A., \& Jones, C. A. 2006, ApJ, 653, 765

Saio, H., Kato, M., \& Nomoto, K. 1988a, ApJ, 331, 388

Saio, H., Nomoto, K., \& Kato, M. 1988b, Natur, 334, 508

Sawai, H., Kotake, K., \& Yamada, S. 2005, ApJ, 631, 446

Sawai, H., \& Yamada, S. 2016, ApJ, 817, 153

Sawai, H., Yamada, S., Kotake, K., \& Suzuki, H. 2013, ApJ, 764, 10

Scheck, L., Janka, H.-T., Foglizzo, T., \& Kifonidis, K. 2008, A\&A, 477, 931

Scheck, L., Kifonidis, K., Janka, H.-T., \& Müller, E. 2006, A\&A, 457, 963

Scheck, L., Plewa, T., Janka, H.-T., Kifonidis, K., \& Müller, E. 2004, PhRvL, 92, 011103

Sedov, L. I. 1959, Similarity and Dimensional Methods in Mechanics (New York: Academic Press)

Shigeyama, T., \& Nomoto, K. 1990, ApJ, 360, 242

Silva Aguirre, V., Ballot, J., Serenelli, A. M., \& Weiss, A. 2011, A\&A, 529, A63

Sinnott, B., Welch, D. L., Rest, A., Sutherland, P. G., \& Bergmann, M. 2013, ApJ, 767, 45

Smartt, S. J. 2009, ARA\&A, 47, 63

Smith, N. 2007, AJ, 133, 1034

Smith, N., \& Arnett, W. D. 2014, ApJ, 785, 82

Socrates, A., Blaes, O., Hungerford, A., \& Fryer, C. L. 2005, ApJ, 632, 531

Spruit, H. C. 1992, A\&A, 253, 131

Spruit, H. C. 2013, A\&A, 552, A76

Stathakis, R. A., Dopita, M. A., Cannon, R. D., \& Sadler, E. M. 1991, in Supernovae, ed. S. E. Woosley (New York: Springer-Verlag), 95

Sugerman, B. E. K., Crotts, A. P. S., Kunkel, W. E., Heathcote, S. R., \& Lawrence, S. S. 2005a, ApJ, 627, 888

Sugerman, B. E. K., Crotts, A. P. S., Kunkel, W. E., Heathcote, S. R., \& Lawrence, S. S. 2005b, ApJS, 159, 60

Sukhbold, T., Ertl, T., Woosley, S. E., Brown, J. M., \& Janka, H.-T. 2016, ApJ, 821,38

Sunyaev, R., Kaniovsky, A., Efremov, V., et al. 1987, Natur, 330, 227

Suwa, Y., Kotake, K., Takiwaki, T., et al. 2010, PASJ, 62, L49

Takiwaki, T., Kotake, K., \& Sato, K. 2009, ApJ, 691, 1360

Takiwaki, T., Kotake, K., \& Suwa, Y. 2012, ApJ, 749, 98

Takiwaki, T., Kotake, K., \& Suwa, Y. 2014, ApJ, 786, 83
Timmes, F. X., \& Swesty, F. D. 2000, ApJS, 126, 501

Tziamtzis, A., Lundqvist, P., Gröningsson, P., \& Nasoudi-Shoar, S. 2011, A\&A, 527, A35

Urushibata, T., Takahashi, K., Umeda, H., \& Yoshida, T. 2018, MNRAS, 473, L101

Utrobin, V. P., Chugai, N. N., \& Andronova, A. A. 1995, A\&A, 295, 129

Utrobin, V. P., Wongwathanarat, A., Janka, H.-T., et al. 2019, A\&A, 624, A116

Utrobin, V. P., Wongwathanarat, A., Janka, H.-T., \& Müller, E. 2015, A\&A, 581, A40

Varani, G. F., Meikle, W. P. S., Spyromilio, J., \& Allen, D. A. 1990, MNRAS 245,570

Vartanyan, D., Burrows, A., Radice, D., Skinner, M. A., \& Dolence, J. 2019, MNRAS, 482, 351

Viallet, M., Meakin, C., Arnett, D., \& Mocák, M. 2013, ApJ, 769, 1

Viallet, M., Meakin, C., Prat, V., \& Arnett, D. 2015, A\&A, 580, A61

Walborn, N. R., Lasker, B. M., Laidler, V. G., \& Chu, Y.-H. 1987, ApJL, 321, L41

Wampler, E. J., Wang, L., Baade, D., et al. 1990, ApJL, 362, L13

Weaver, T. A., Zimmerman, G. B., \& Woosley, S. E. 1978, ApJ, 225, 1021

Weiss, A., Hillebrandt, W., \& Truran, J. W. 1988, A\&A, 197, L11

West, R. M., Lauberts, A., Jorgensen, H. E., \& Schuster, H. E. 1987, A\&A 177, L1

Wongwathanarat, A., Janka, H.-T., \& Müller, E. 2010, ApJL, 725, L106

Wongwathanarat, A., Janka, H.-T., \& Müller, E. 2013, A\&A, 552, A126

Wongwathanarat, A., Müller, E., \& Janka, H.-T. 2015, A\&A, 577, A48

Woosley, S. E. 1987, in IAU Symp. 125, The Origin and Evolution of Neutron Stars, ed. D. J. Helfand \& J.-H. Huang (Dordrecht: Reidel), 255

Woosley, S. E. 1988, ApJ, 330, 218

Woosley, S. E., Heger, A., \& Weaver, T. A. 2002, RvMP, 74, 1015

Woosley, S. E., Pinto, P. A., \& Ensman, L. 1988, ApJ, 324, 466

Xiong, D.-R. 1977, AcASn, 18, 86

Xiong, D. R., Cheng, Q. L., \& Deng, L. 1997, ApJS, 108, 529

Yamada, S., \& Sato, K. 1991, ApJ, 382, 594

Yoshida, T., Takiwaki, T., Kotake, K., et al. 2019, ApJ, 881, 16

Zaussinger, F., \& Spruit, H. C. 2013, A\&A, 554, A119

Zhang, Q. S. 2013, ApJS, 205, 18

Zhang, Q. S. 2016, ApJ, 818, 146 